\title{
CALIFA, the Calar Alto Legacy Integral Field Area survey
}

\section{Survey presentation ${ }^{\star}$}

\author{
S. F. Sánchez ${ }^{1}$, R. C. Kennicutt ${ }^{2}$, A. Gil de Paz ${ }^{3}$, G. van de Ven ${ }^{4}$, J. M. Vílchez ${ }^{5}$, L. Wisotzki ${ }^{6}$, C. J. Walcher ${ }^{6}$, \\ D. Mast ${ }^{5,1}$, J. A. L. Aguerri ${ }^{9,28}$, S. Albiol-Pérez ${ }^{20}$, A. Alonso-Herrero ${ }^{12}$, J. Alves ${ }^{22}$, J. Bakos ${ }^{9,28}$, T. Bartáková34, \\ J. Bland-Hawthorn $^{7}$, A. Boselli ${ }^{19}$, D. J. Bomans ${ }^{25}$, A. Castillo-Morales ${ }^{3}$, C. Cortijo-Ferrero ${ }^{5}$, \\ A. de Lorenzo-Cáceres ${ }^{9,28}$, A. del Olmo ${ }^{5}$, R.-J. Dettmar ${ }^{25}$, A. Díaz ${ }^{10}$, S. Ellis ${ }^{8,7}$, J. Falcón-Barroso ${ }^{9,28}$, H. Flores ${ }^{31}$, \\ A. Gallazzi ${ }^{14}$, B. García-Lorenzo ${ }^{9,28}$, R. González Delgado ${ }^{5}$, N. Gruel ${ }^{24}$, T. Haines ${ }^{26}$, C. Hao $^{32}$, B. Husemann $^{6}$, \\ J. Iglésias-Páramo ${ }^{5,1}$, K. Jahnke ${ }^{4}$, B. Johnson ${ }^{30}$, B. Jungwiert ${ }^{16,33}$, V. Kalinova ${ }^{4}$, C. Kehrig ${ }^{6}$, D. Kupko ${ }^{6}$, \\ Á. R. López-Sánchez ${ }^{8,23}$, M. Lyubenova ${ }^{4}$, R. A. Marino ${ }^{1,3}$, E. Mármol-Queraltó ${ }^{1,3}$, I. Márquez ${ }^{5}$, J. Masegosa ${ }^{5}$, \\ S. Meidt ${ }^{4}$, J. Mendez-Abreu ${ }^{9,28}$, A. Monreal-Ibero ${ }^{5}$, C. Montijo ${ }^{5}$, A. M. Mourão ${ }^{17}$, G. Palacios-Navarro ${ }^{21}$, \\ P. Papaderos ${ }^{15}$, A. Pasquali ${ }^{29}$, R. Peletier ${ }^{11}$, E. Pérez ${ }^{5}$, I. Pérez ${ }^{27}$, A. Quirrenbach ${ }^{13}$, M. Relaño ${ }^{27}$, \\ F. F. Rosales-Ortega ${ }^{10,1}$, M. M. Roth ${ }^{6}$, T. Ruiz-Lara ${ }^{27}$, P. Sánchez-Blázquez ${ }^{10}$, C. Sengupta ${ }^{1,5}$, R. Singh $^{4}$, \\ V. Stanishev ${ }^{17}$, S. C. Trager ${ }^{11}$, A. Vazdekis ${ }^{9,28}$, K. Viironen ${ }^{1,24}$, V. Wild ${ }^{18}$, S. Zibetti ${ }^{14}$, and B. Ziegler ${ }^{22}$ \\ (Affiliations can be found after the references)
}

Received 27 May 2011 / Accepted 3 November 2011

\begin{abstract}
The final product of galaxy evolution through cosmic time is the population of galaxies in the local universe. These galaxies are also those that can be studied in most detail, thus providing a stringent benchmark for our understanding of galaxy evolution. Through the huge success of spectroscopic single-fiber, statistical surveys of the Local Universe in the last decade, it has become clear, however, that an authoritative observational description of galaxies will involve measuring their spatially resolved properties over their full optical extent for a statistically significant sample. We present here the Calar Alto Legacy Integral Field Area (CALIFA) survey, which has been designed to provide a first step in this direction. We summarize the survey goals and design, including sample selection and observational strategy. We also showcase the data taken during the first observing runs (June/July 2010) and outline the reduction pipeline, quality control schemes and general characteristics of the reduced data.

This survey is obtaining spatially resolved spectroscopic information of a diameter selected sample of $\sim 600$ galaxies in the Local Universe $(0.005<z<0.03)$. CALIFA has been designed to allow the building of two-dimensional maps of the following quantities: (a) stellar populations: ages and metallicities; (b) ionized gas: distribution, excitation mechanism and chemical abundances; and (c) kinematic properties: both from stellar and ionized gas components. CALIFA uses the PPAK integral field unit (IFU), with a hexagonal field-of-view of $\sim 1.3 \square$, with a 100\% covering factor by adopting a three-pointing dithering scheme. The optical wavelength range is covered from 3700 to $7000 \AA$, using two overlapping setups (V500 and V1200), with different resolutions: $R \sim 850$ and $R \sim 1650$, respectively. CALIFA is a legacy survey, intended for the community. The reduced data will be released, once the quality has been guaranteed.

The analyzed data fulfill the expectations of the original observing proposal, on the basis of a set of quality checks and exploratory analysis: (i) the final datacubes reach a $3 \sigma$ limiting surface brightness depth of $\sim 23.0 \mathrm{mag} / \operatorname{arcsec}^{2}$ for the V500 grating data $\left(\sim 22.8 \mathrm{mag} / \mathrm{arcsec}^{2}\right.$ for V1200); (ii) about $\sim 70 \%$ of the covered field-of-view is above this $3 \sigma$ limit; (iii) the data have a blue-to-red relative flux calibration within a few percent in most of the wavelength range; (iv) the absolute flux calibration is accurate within $\sim 8 \%$ with respect to SDSS; (v) the measured spectral resolution is $\sim 85 \mathrm{~km} \mathrm{~s}^{-1}$ for V1200 ( 150 $\mathrm{km} \mathrm{s}^{-1}$ for V500); (vi) the estimated accuracy of the wavelength calibration is $\sim 5 \mathrm{~km} \mathrm{~s}^{-1}$ for the V1200 data ( $\sim 10 \mathrm{~km} \mathrm{~s}^{-1}$ for the V500 data); (vii) the aperture matched CALIFA and SDSS spectra are qualitatively and quantitatively similar. Finally, we show that we are able to carry out all measurements indicated above, recovering the properties of the stellar populations, the ionized gas and the kinematics of both components. The associated maps illustrate the spatial variation of these parameters across the field, reemphasizing the redshift dependence of single aperture spectroscopic measurements. We conclude from this first look at the data that CALIFA will be an important resource for archaeological studies of galaxies in the Local Universe.
\end{abstract}

Key words. techniques: spectroscopic - galaxies: active - galaxies: evolution - surveys - galaxies: ISM - galaxies: stellar content

\section{Introduction}

Our understanding of the Universe and its constituents comes from large surveys such as the 2dFGRS (Folkes et al. 1999),

* Based on observations collected at the Centro Astronómico Hispano Alemán (CAHA) at Calar Alto, operated jointly by the MaxPlanck-Institut für Astronomie and the Instituto de Astrofísica de Andalucía (CSIC).
SDSS (York et al. 2000), GEMS (Rix et al. 2004), VVDS (Le Fèvre et al. 2004), and COSMOS (Scoville et al. 2007), to name but a few. Such surveys have not only constrained the evolution of global quantities such as the cosmic star formation rate, but also enabled us to link these with the properties of individual galaxies - morphological types, stellar masses, metallicities, etc. Compared to previous approaches, the major advantages of this recent generation of surveys are: (1) the large number of 
objects sampled, allowing for meaningful statistical analysis to be performed on an unprecedented scale; (2) the possibility to construct large comparison/control samples for each subset of galaxies; (3) a broad coverage of galaxy subtypes and environmental conditions, allowing for the derivation of universal conclusions; and (4) the homogeneity of the data acquisition, reduction and (in some cases) analysis.

On the other hand, the cost of these surveys, in terms of telescope time, person-power, and time scales involved, is also unprecedented in astronomy. The user of such data products has not necessarily been involved in any step of designing or conducting the survey, but nevertheless takes advantage of the data by exploiting them according to her/his scientific interests. This new approach to observational astronomy is also changing our perception of the scientific rationale behind a new survey: while it is clear that certain planned scientific applications are key determinants to the design of the observations and "drive" the survey, the survey data should, at the same time, allow for a broad range of scientific exploitation. This aspect is now often called a survey's legacy value.

Current technology generally leads to surveys either in the imaging or in the spectroscopic domain. While imaging surveys provide two-dimensional coverage, the information content of a photometric spectral energy distribution (SED) is limited. This remains true for the new generation of multi-band photometric surveys such as COMBO-17 (Wolf et al. 2003), ALHAMBRA (Moles et al. 2008), the planned PAU project (Benítez et al. 2009), COSMOS (Scoville et al. 2007) or the LUS survey ${ }^{1}$, which are geared towards better precision in redshift, mean ages and stellar masses, but are nevertheless unable to measure individual spectral lines and thus emission line ratios or internal radial velocity differences. Spectroscopic surveys such as SDSS or zCOSMOS (Lilly et al. 2007), on the other hand, do provide more detailed astrophysical information, but they are generally limited to one spectrum per galaxy. One thus misses all information on the radial distribution of galaxy properties and on all details of the kinematics. Even when attempting to describe galaxies by their integrated properties only, this state of affairs also leads to aperture losses that are difficult to control. For example, the 3 " diameter of the fiber used in the SDSS corresponds to different physical scales at different redshifts, with limited possibilities to correct for these aperture effects (e.g., Kewley et al. 2005; Ellis et al. 2005). The most popular method is to compare results of a fit to the photometry of the whole galaxy with the photometric fit corresponding to the area of the fiber only (Gómez et al. 2003; Brinchmann et al. 2004). Even more severely, Zibetti et al. (2009) have recently shown that spatially resolved stellar population analysis may lead to corrections of up to $40 \%$ for the stellar mass of a galaxy, when compared to integrated light studies.

An observational technique combining the advantages of imaging and spectroscopy (albeit with usually quite small field of view) is integral field spectroscopy (IFS). This technique allows us to study both the integrated and spatially resolved spectroscopic properties of galaxies. IFS has the potential to provide observational evidence to constrain many outstanding questions of baryonic physics which are key to our understanding of galaxy evolution and, therefore, cosmology. Some of these are (1) the importance and consequences of merging, major and minor; (2) internal dynamical processes, such as bars, spiral arms, stellar migration; (3) environmental effects, such as tidal forces, stripping; (4) AGN feedback; (5) occurrence, spatial and temporal extent and trigger of star formation. Spatially resolved

\footnotetext{
1 http://www.inaoep.mx/ gtc-lus/
}

spectroscopic properties of a statistical sample of nearby galaxies is the dataset required to address these questions.

However, so far, IFS has rarely been used in a "survey mode" to investigate sizeable samples. Among the few exceptions there is, most notably, the SAURON survey (de Zeeuw et al. 2002), focused on the study of the central regions of 72 nearby earlytype galaxies and bulges of spirals, and its extension ATLAS ${ }^{3 \mathrm{D}}$ (260 early-type galaxies at $z<0.01$; Cappellari et al. 2011). Others are the on-going PINGS project (Rosales-Ortega et al. $2010)$ at the CAHA $3.5 \mathrm{~m}$ of a dozen of very nearby galaxies $(\sim 10 \mathrm{Mpc})$ and the currently ongoing study of 70 (U)LIRGS at $z<0.26$ using different IFUs (Arribas et al. 2008). Finally, the VIRUS-P instrument is currently used to carry out two small IFS surveys, namely VENGA (30 spiral galaxies, Blanc et al. 2010) and VIXENS ${ }^{2}$ (15 starbursts). All these datasets are clearly focused on specific science questions, adopting correspondingly optimized sample selection criteria and also observing strategies. For example, at the redshifts of the galaxies in the ATLAS $^{3 D}$ sample SAURON has a field of view of $30^{\prime \prime} \times 40^{\prime \prime}$, or $<7 \times 9 \mathrm{kpc}$, thus does not cover the outer parts of these galaxies.

On completion, CALIFA will be the largest and the most comprehensive wide-field IFU survey of galaxies carried out to date. It will thus provide an invaluable bridge between large single aperture surveys and more detailed studies of individual galaxies. With CALIFA we will fix observational properties of galaxies in the Local Universe, which will have a potential impact in the interpretation of observed properties at higher redshift (e.g. Epinat et al. 2010).

CALIFA is an ongoing survey, which has been granted with 210 dark nights by the Calar Alto Executive Committee, spanning through 6 semesters. The gathering of the data started in June/July 2010, and after a technical problem with the telescope, it was resumed in March 2011. This technical problem, fully repaired, has not affected the quality of the CALIFA data. About 20 galaxies are observed per month. As mentioned above, there will be consecutive data releases of the fully reduced datasets, once certain milestones/number of observed galaxies are reached. The first one is planned for late 2012, when a total number of 100 galaxies is completed (i.e., observed, reduced and quality of the data tested). The current status of data acquisition can be obtained from the CALIFA webpage ${ }^{3}$.

In this article we present the main characteristics of the survey, starting from the design requirements in Sect. 2. Section 3 describes the sample selection criteria, and the main characteristics of the CALIFA mother sample. In Sect. 4 we describe the observing strategy, in particular the observations performed during the first CALIFA runs. The data reduction is described in detail in Sect. 5, and some of the first quality tests performed on the data are presented in Sect. 6. The exploratory analysis performed on these first datasets, obtained in 2010, to verify that we will be able to reach our science goals are presented in Sect. 7. A summary and conclusion of the results is presented in Sect. 8.

\section{Design drivers}

CALIFA has been designed to increase our knowledge of the baryonic physics of galaxy evolution. We intend to characterize observationally the local galaxy population with the following key points in mind:

- Sample covering a substantial fraction of the galaxy luminosity function.

\footnotetext{
2 http://www.as.utexas. edu/ alh/vixens.html

3 http://www. caha.es/CALIFA/
} 
- Large enough sample to allow statistically significant conclusions for all classes of galaxies represented in the survey.

- Characterization of galaxies over their full spatial extent (covering in most cases the R25 radius), i.e. avoiding aperture biases and harnessing the additional power of 2D resolution (gradients, sub-structures: bars, spiral arms...).

- Measurement of gas ionization mechanisms: star formation, shocks, AGN.

- Measurement of ionized gas oxygen and nitrogen abundances.

- Measurement of stellar population properties: ages, mass-tolight ratios, metallicities, and (to a limited extent) abundance patterns.

- Measurement of galaxy kinematics in gas and stars, i.e. velocity fields for all galaxies and velocity dispersions for the more massive ones.

A careful assessment of these, partially competing, drivers and of the practical limits imposed by the instrument, the observatory and the timescale has led to the following key characteristics of the survey: (i) Sample: $\sim 600$ galaxies of any kind in the Local Universe, covering the full color-magnitude diagram down to $M_{B} \sim-18 \mathrm{mag}$, selected from the SDSS to allow good a priori characterization of the targets; (ii) Instrument: PPAK IFU of the Potsdam Multi-Aperture Spectrograph (PMAS) instrument at the $3.5 \mathrm{~m}$ telescope of CAHA, with one of the largest fieldsof-view for this kind of instruments $\left(>1 \operatorname{arcmin}^{2}\right)$; (iii) Grating setups: two overlapping setups, one in the red (3750-7000 $\AA$, $R \sim 850$, for ionized gas measurements and stellar populations) and one in the blue (3700-4700 $\AA, R \sim 1650$, for detailed stellar populations and for stellar and gas kinematics); and (iii) Exposure times: $1800 \mathrm{~s}$ in the blue and $900 \mathrm{~s}$ in the red.

\section{The CALIFA galaxy sample}

We now briefly describe how the sample has been constructed. We limit this section to some fundamental considerations that are important for understanding the potential and limitations of the survey. In a later paper we will present a detailed characterization of the sample and the distribution of galaxy properties, including comparisons to other studies and the galaxy population as a whole.

The guiding principle for building the CALIFA galaxy sample followed from the broad set of scientific aims outlined in the previous section, i.e. the sample should cover a wide range of important galaxy properties such as morphological types, luminosities, stellar masses, and colours. Further constraints were added due to technical and observational boundary conditions, such as observing all galaxies with the same spectroscopic setup to make scheduling as flexible as possible. As detailed below, a small set of selection criteria was employed to define a sample of 937 possible target galaxies, the so-called "CALIFA mother sample". From this mother sample, the galaxies are drawn as actual targets only according to their visibility, i.e. in a quasirandom fashion, so that the final catalog of CALIFA galaxies will be a slightly sparsely sampled $(\sim 2 / 3)$ subset of the mother sample.

The CALIFA mother sample was initially selected from the SDSS DR7 photometric catalog (Abazajian et al. 2009), which ensures the availability of good quality multi-band photometry, and in many cases (but not all, cf. below) nuclear spectra. The CALIFA footprint is thus largely identical to that of the SDSS imaging survey, modified only by an additional restriction of $\delta>7^{\circ}$ for galaxies in the North Galactic hemisphere to ensure

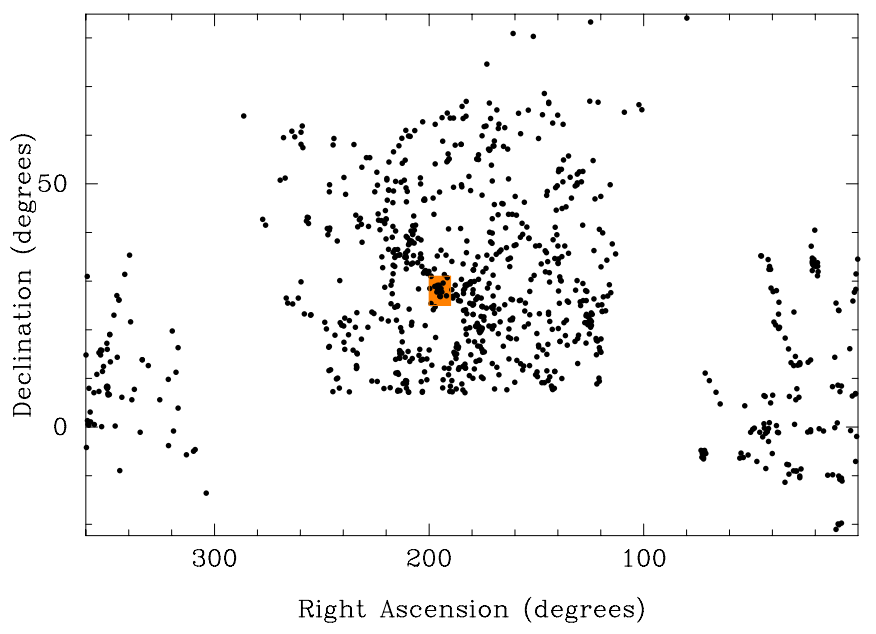

Fig. 1. Distribution of the targets in the CALIFA mother sample in equatorial coordinates. The distribution over the two Galactic caps is obvious. Note that the north galactic plane part of the sample has been limited to $\delta>+7^{\circ}$ while the south galactic plane part does not have that limitation, to counterbalance the much lower number of SDSS objects in the south galactic plane region. The orange square at the center of the figure indicates the location of the Coma cluster.

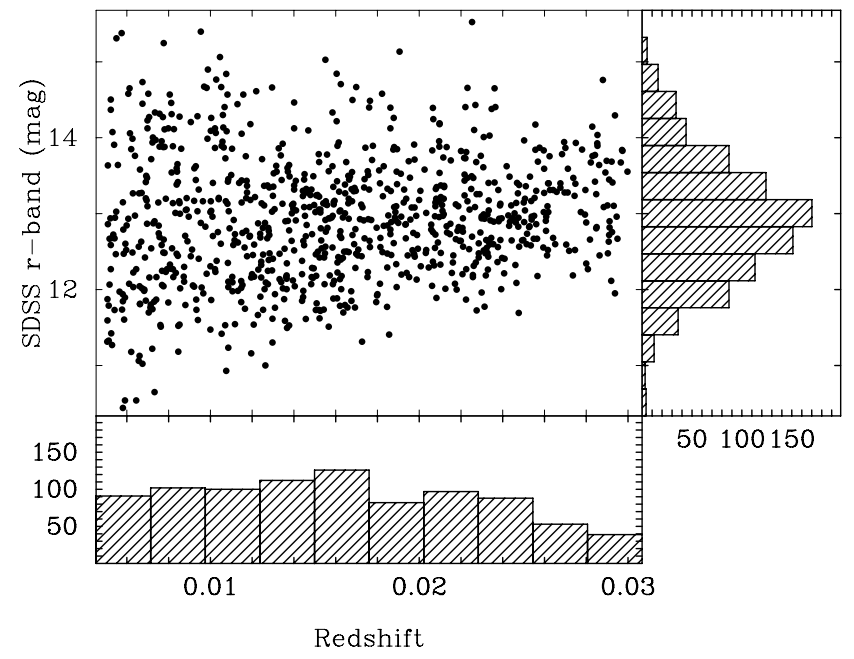

Fig. 2. Apparent $r$-band magnitude and redshift distribution of the CALIFA mother sample.

good visibility from the observatory. Note that this criterion was not applied to objects at southern Galactic latitudes, in order to at least partly counterbalance the much smaller sky coverage of SDSS data in this region and improve on the observability of CALIFA targets throughout the year. Figure 1 shows the resulting distribution of the CALIFA mother sample in the sky.

The defining selection criteria of the CALIFA mother sample comprise a combination of angular isophotal diameter selection with lower and upper redshift limits $(0.005<z<0.03)$. We further justify and discuss the consequences of these selection criteria later in this section. Note that while no explicit cut in apparent magnitude is involved, the adopted criteria implicitly ensure that only relatively bright galaxies enter the sample, with a roughly Gaussian distribution of the total $r$-band magnitudes centred on a mean of $\bar{r}=13.3$ and with a $1 \sigma$ dispersion of $0.8 \mathrm{mag}$. No CALIFA galaxy is fainter than $r=16$. In Fig. 2 we show the distribution of the mother sample in the fundamental observed properties, total $r$-band magnitudes and redshifts. 
The choice of redshift range was driven by two requirements: (1) objects within the luminosity range of interest should have apparent sizes well matched to the PPAK field-of-view (FoV); (2) all relevant emission lines in all galaxies should be covered with a single spectral setup. As measure of the apparent galaxy size we adopted the "isoA_r" values (isophotal diameters $D_{25}$ in the SDSS $r$-band) and selected only galaxies with $45^{\prime \prime}<D_{25}<80^{\prime \prime}$. We demonstrate below in Sect. 6.5 that this strategy indeed allows for a very efficient usage of the instrument, in the sense that a large fraction of the FoV provides useful data. The upper limit in the apparent diameters furthermore ensures that the contribution of light from the galaxy at the position of the sky fibers is negligible (typically $>27.5 \mathrm{mag} \operatorname{arcsec}^{2}$ in the $r$-band). We thus avoid objects larger than the FoV, which would require a mosaicing strategy plus separate sky exposures leading to a dramatic reduction of the observing efficiency in terms of the number of galaxies observable within a given time.

Not all galaxies in the SDSS photometric catalog obeying our isophotal diameter criterion have spectra - and therefore redshifts - in the SDSS spectroscopic database, which is known to become increasingly incomplete for total magnitudes brighter that $r \sim 14$. In order to (as much as possible) overcome such an undesirable bias against bright galaxies, we supplemented the SDSS redshifts with information accessed through the SIMBAD database at CDS, which in turn is a compilation of a large variety of observations and redshift catalogs. Hence, there are SDSSbased spectra and redshifts for $\sim 60 \%$ of the CALIFA galaxies, while for the remainder we have only the redshift information provided by SIMBAD. As the latter is also not $100 \%$ complete, there could be a few galaxies within the CALIFA footprint without redshift measurements and, therefore, outside of the CALIFA mother sample. It is difficult to quantify this incompleteness, but it is unlikely to be more than a few percent, and probably much less.

Our decision to construct a diameter-selected sample has several practical advantages, besides the obvious benefit of efficiently using the instrumental field-of-view. Another advantage has already been mentioned: for the adopted redshift range, the distribution of apparent galaxy magnitudes naturally favours relatively bright systems, and in fact there was no need to define an additional faint flux limit to the survey (see Fig. 2). Furthermore, the range in absolute magnitudes is considerably broadened due to the factor of 6 between lowest and highest redshifts, so that the CALIFA sample encompasses an interval of $>7$ mag in intrinsic luminosities. In fact, the low-redshift cutoff was mainly introduced in order to limit the luminosity range and avoid swamping the sample with dwarf galaxies, which - given the limitation to 600 galaxies in total - were considered to be outside the main scientific interest of the CALIFA project.

Together with the wide range in luminosities comes a broad coverage of galaxy colours, and the CALIFA sample includes substantial numbers of galaxies in all populated areas of the colour-magnitude diagram, from the red sequence through the green valley to the blue cloud (which is of course effectively truncated at its faint end due to the low-redshift limit). This broad colour distribution is illustrated in Fig. 3, where we compare the $u-z$ vs. $M_{z}$ relation of galaxies in the CALIFA mother sample with the corresponding distribution in the SDSS-NYU catalog (e.g. Blanton et al. 2005). While there are (and should be) differences in the details of the distribution - which can be quantified, see below -, it is immediately clear that CALIFA at least qualitatively represents a wide range of galaxy types. Figure 3 also provides the number of CALIFA objects per bin in $M_{z}$ and $u-z$ (recall, however, that the observed sample size

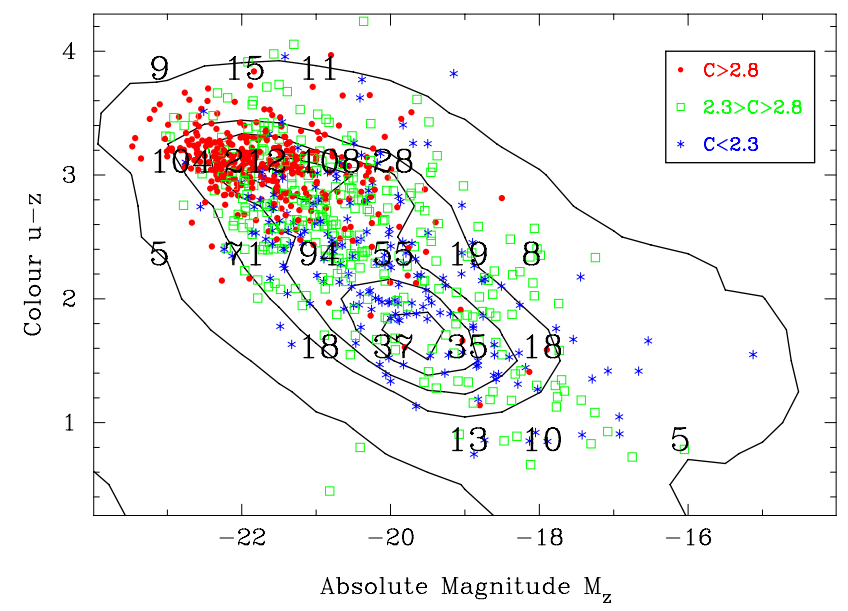

Fig. 3. Distribution of the CALIFA mother sample in the $u-z$ vs. $M_{z}$ colour-magnitude diagram. The overplotted numbers indicate the number of galaxies in bins of $1 \mathrm{mag}$ in $M_{r}$ and $0.75 \mathrm{mag}$ in $u-z$. Different colours and symbols represent a classification into bulge- and diskdominated galaxies as well as intermediate cases, as suggested by the concentration index $C$. For comparison, the contours delineate the number density distribution of galaxies in the SDSS-NYU catalogue (e.g., Blanton et al. 2005).

will be smaller by a factor $\sim 2 / 3$ ). These numbers show that there will be sufficient statistics in several bins to make robust statements about typical galaxy properties, for early-type as well as late-type galaxies. In fact, these were the numbers that drove the overall time request for CALIFA to enable a total sample size of 600 galaxies.

The broad representation of galaxy properties in the CALIFA sample is also reflected in the distribution of morphological types. While a thorough characterization of the sample in terms of morphology and structural properties will be the subject of a future paper, a qualitative impression can be obtained already from rather simple diagnostics. It has been demonstrated in the past (Strateva et al. 2001) that bulge- and disk-dominated systems can be reasonably well distinguished by their concentration indices, defined as the ratio $C$ of the $r_{90}$ and $r_{50}$ Petrosian radii provided by the SDSS photometric catalog. Typically, a value of $C \gtrsim 2.8$ requires the presence of a substantial bulge, whereas $C \lesssim 2.3$ is indicative of an exponential disk. In Fig. 3 we coded the symbols into three groups, including a class of transition or uncertain objects with $2.3<C<2.8$. Their different distributions in the colour-magnitude diagram is immediately apparent.

Clearly, the majority of CALIFA galaxies $(\sim 2 / 3)$ have substantial disk components, including irregulars and interacting galaxies, and are more or less actively forming stars. The final sample of $\sim 400$ of such galaxies will clearly exceed any previous IFU study by a large factor. Interestingly, the ominous "green valley" intermediate to star-forming and passive galaxies is well covered by the sample.

On the other hand, CALIFA will also provide IFU data for some 200 bulge-dominated, morphologically early-type galaxies, most of which - as expected - cluster very strongly along the red sequence. While the successful ATLAS ${ }^{3 \mathrm{D}}$ project (Cappellari et al. 2011) has already observed an even somewhat larger number of early-type galaxies, CALIFA will complement the insights from ATLAS ${ }^{3 \mathrm{D}}$ due to its much larger spectral coverage $(\sim 6 \times)$ and FoV, which will allow the study of the outer regions of early-type galaxies.

In terms of environment, the CALIFA sample is clearly dominated by field galaxies. It will effectively include galaxy 

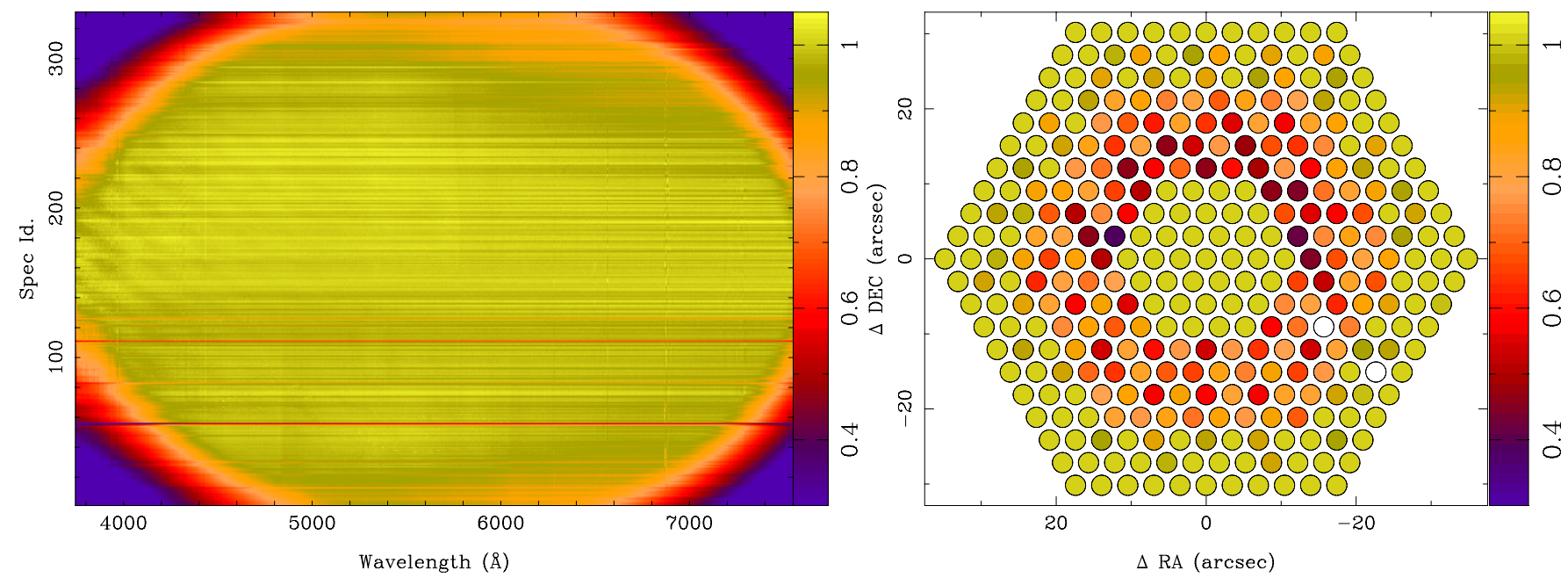

Fig. 4. Left panel: intensity map of the differential transmission fiber-to-fiber for the V500-grating (known as fiber-flat), corresponding to the night of the 10th of June 2010. The effects of vignetting are clearly visible with a significant drop of the transmission at the edges of the image. Right panel: spatial distribution of the fraction of pixels free of vignetting, when adopting a conservative criterion of $75 \%$ of the average transmission in the central fibers. Two fibers at the bottom/right show a transmission below this limit in the whole wavelength range.

populations in groups, but much denser environments will be poorly sampled. A rough estimate of the total number of galaxy clusters in the sample can be obtained from the counts by Baillard et al. (2011), leading to a maximum of 10 clusters that will, at least partially, be covered by CALIFA. Fortunately, the Coma cluster at $z \simeq 0.023$ is fully covered by the CALIFA footprint and redshift range (cf. Fig. 1). Therefore, there will be some (limited) ability to study the environmental dependence of galaxy properties.

Any sample of galaxies faces the question about its ability to represent, in a statistically well-defined way, the properties of the galaxy population as a whole. In some cases, e.g. in the ATLAS ${ }^{3 \mathrm{D}}$ project, it has been possible to construct and observe strictly volume-limited samples for which this relation can be directly made, but this is not a viable approach for a more generic survey covering a wide range of luminosities. Many surveys are simply flux-limited (such as the SDSS main galaxy sample), possibly supplemented by an additional volume limit imposed by redshift cuts, and statistical relations are then obtained by applying volume corrections to the apparent trends. One of the advantages of working with a diameter-limited sample as in CALIFA is the fact that volume corrections can then be applied just as easily as for flux-limited samples (see, Davies et al. 1990; de Jong \& van der Kruit 1995, for extensive discussions of diameter-limited samples and volume correction). We will always use such corrections for CALIFA when deriving statistical trends within the galaxy population.

Of course, our ability to perform volume corrections does not imply that the CALIFA sample is free from selection biases. Such biases may even occur directly as a consequence of the selection procedure. For example, the low-redshift limit of $z>0.005$ causes low-luminosity galaxies to be missing from the CALIFA sample, with incompleteness setting in roughly around $M_{r} \sim-20$ and becoming severe for $M_{r} \gtrsim-18$. This type of bias is unavoidable when applying explicit redshift cuts. The degree of incompleteness can then be estimated by comparison with samples selected by other criteria. Nevertheless, there may also be more subtle biases affecting our sample that we are currently not aware of, and we will investigate this issue further. We also continuously check that the selection of CALIFA galaxies for observation - supposed to be essentially random, since it is based only on visibility during a given night - does not introduce spurious trends incompatible with the full sample.

As already mentioned, a more detailed characterization of the CALIFA mother sample will be presented in a separate article that is currently being prepared. That paper will provide a detailed analysis of the distribution of morphological and multicolour properties, estimates of the survey selection function for several parameters, and in particular a compilation of archival multi-wavelength information.

\section{Observing strategy}

The observations of the CALIFA survey officially started in July 2010, being performed at the $3.5 \mathrm{~m}$ telescope of the Calar Alto observatory with the Potsdam Multi Aperture Spectrograph, PMAS (Roth et al. 2005) in the PPAK mode (Verheijen et al. 2004; Kelz et al. 2006). The PPAK fiber bundle consists of 382 fibers of 2.7 arcsec diameter each (see Fig. 5 in Kelz et al. 2006). Of these 382 fibers, 331 (the science fibers) are concentrated in a single hexagonal bundle covering a field-of-view of $74^{\prime \prime} \times 64^{\prime \prime}$, with a filling factor of $\sim 60 \%$. The sky background is sampled by 36 additional fibers, distributed in 6 bundles of 6 fibers each, along a circle $\sim 72$ arcsec from the center of the instrument FoV. The sky-fibers are distributed among the science fibers within the pseudo-slit in order to have a good characterization of the sky, sampled with a similar distortion than the science fibers; the remaining 15 fibers are used for calibration purposes. Cross-talk between the adjacent spectra in the detector is estimated to be less than $5 \%$ when using a pure aperture extraction (Sánchez 2006a). Adjacent spectra on the CCD may be mapped to very different locations in the spatial plane (Kelz et al. 2006). However, it introduces an incoherent contamination, not important for the present study, that is minimized by adopting a more refined extraction procedure during the reduction process.

PMAS was upgraded with an E2V CCD231 $4 \mathrm{~K} \times 4 \mathrm{~K}$ in October 2009 (Roth et al. 2010), which has increased nominally the wavelength range covered by a certain instrumental setup by a factor two, with respect to the values reported by Roth et al. (2005). However, this nominal increase is not fully met at the 


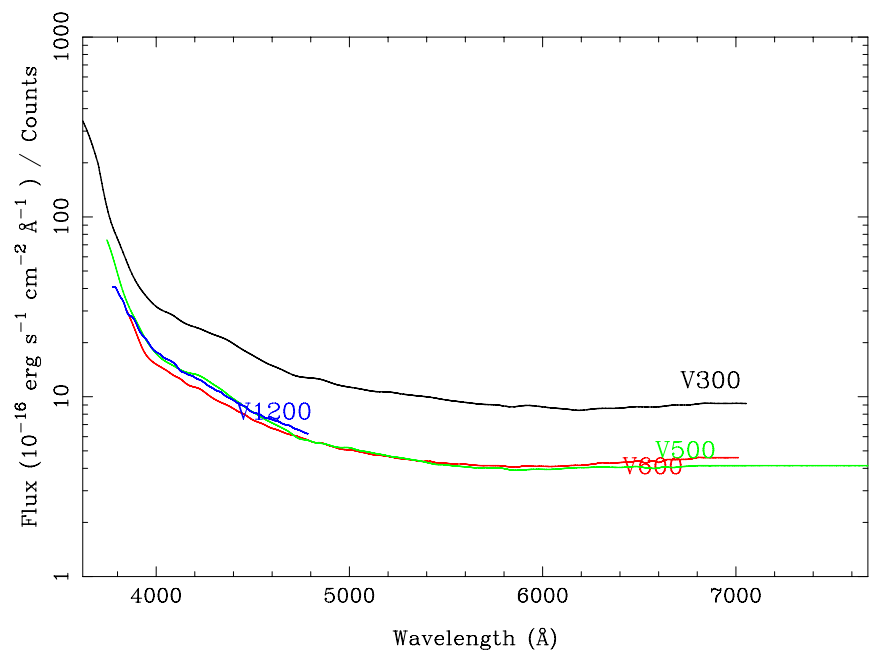

Fig. 5. Flux density-to-counts ratio for the gratings used in the CALIFA survey, derived for the most transparent night of those observed so far, as compared to the most frequently used gratings for PMAS (V300 and V600).

four corners of the CCD. As a result of a trade-off study for the PMAS fiber spectrograph, the optical system was deliberately designed to tolerate some vignetting at the edges of the full detector field-of-view, as reported by Roth et al. (1997), and Roth et al. (1998). Figure 4 illustrates the effect of vignetting for the new $4 \mathrm{~K} \times 4 \mathrm{~K} \mathrm{CCD}$, which was not noticeable in the previous $2 \mathrm{~K} \times 4 \mathrm{~K}$ detector configuration. The left panel shows a map of the differential fiber-to-fiber transmission for all the 331 fibers in the hexagonal fiber-bundle. The effect of the vignetting is clearly identified as a drop in the transmission at the four edges of the map. $70 \%$ of the spectra are free from vignetting. However, $30 \%$ of the spectra, namely those close to the edge of the detector, do suffer some loss of efficiency that gradually increases towards the corners of the CCD. The loss of throughput amounts to up to $30 \%$ in the best case and $50 \%$ in the worst case, for one half of those spectra, but more than $50 \%$ for the other half. It can be seen in Fig. 4 (left panel), that where vignetting sets in, no more than a quarter of the full spectral range is affected. In summary the vignetting severely affects less than a $15 \%$ of the fibers, in less than a $25 \%$ of their wavelength range.

The right panel illustrates the spatial distribution of the vignetting. The mapping of the PPAK fibers from the telescope focal plane to the CCD implies that the areas affected by the vignetting correspond to an annular ring at about $\sim 15^{\prime \prime}$ from the center of the FoV. In addition, we report the existence of two broken fibers with a lower transmission than the average (fibers 65 and 127), not reported before.

A dithering scheme with three pointings has been adopted in order to cover the complete FoV of the central bundle and to increase the spatial resolution of the data. This scheme was already used in previous studies using PPAK (Sánchez et al. 2007b; Castillo-Morales et al. 2010; Pérez-Gallego et al. 2010; Rosales-Ortega et al. 2010). In most of these studies the offsets in RA and DEC of the different pointings, with respect to the nominal coordinates of the targets, were: $0^{\prime \prime}, 0^{\prime \prime} ;+1.56^{\prime \prime},+0.78^{\prime \prime}$, $+1.56^{\prime \prime},-0.78^{\prime \prime}$. These offsets correspond to half the spacing between adjacent fibers, which allows to cover the holes between fibers in the central bundle. However, in order to correctly address the problems generated by the vignetting reported before, we adopted a different dithering scheme for CALIFA, with wider offsets in RA and Dec, i.e.: $0^{\prime \prime}, 0^{\prime \prime}$; $-5.22^{\prime \prime},-4.84^{\prime \prime} ;-5.22^{\prime \prime}$, $+4.84^{\prime \prime}$. These offsets correspond to jumping one fiber to the adjacent hole. This procedure ensures that in the final dithered and rebinned dataset the throughput losses for any spectrum affected by vignetting are compensated through the spectra from at least two adjacent fibers, closer than $2^{\prime \prime}$, which are unaffected by vignetting. A spatial recomposition of the three pointings is included in the standard data reduction scheme.

All objects will be observed using two different and complementary setups. Firstly, the new grating, purchased for CALIFA and named V500, is used. It has a nominal resolution of $\lambda / \Delta \lambda \sim 850$ at $\sim 5000 \AA(F W H M \sim 6 \AA)$, to cover the widest possible wavelength range, nominally from [OII] $\lambda 3727$ to $[\mathrm{SII}] \lambda 6731$ in the rest frame of all objects in the survey. Secondly, the V1200 grating (Roth et al. 2005) is used, with a nominal resolution of $\lambda / \Delta \lambda \sim 1650$ at $\sim 4500 \AA$ $(F W H M \sim 2.7 \AA)$. It covers the blue wavelength range, including [OII] $\lambda 3727$, the Balmer break at $\sim 4000-4400 \AA, \mathrm{H} \delta, \mathrm{H} \gamma$ and [OIII] $\lambda 4363$. The main purpose of the first setup is to study the stellar populations and the properties of the ionized gas using the widest possible baseline of spectral features and emission line species. The observations with the second setup will provide accurate measurements of both the stellar and ionized gas kinematics, mostly through the $\mathrm{H}+\mathrm{K}$ absorption features and the [OII] $\lambda 3727$ emission line, respectively.

In order to avoid strong effects of the described vignetting on the final dataset, the wavelength range covered by each grating was selected in a complementary way. The range covered by the V500 grating was selected such that the reddest emission line of interest ([SII] 16731 ) was free of vignetting in all fibers. The range covered by the V1200 grating was selected such that the bluest emission line of interest ([OII] 23727 ) was free of vignetting. This selection reduces the final wavelength range covered with each grating, but guarantees that all features of interest are unaffected by vignetting in at least one of the setups. A fixed setup was selected for each grating for the whole survey, to guarantee the homogeneity of the dataset. The final nominal wavelength range covered by each grating is 3745-7300 $\AA$ (V500) and 3400-4750 Å (V1200), respectively. However, for the V1200 grating, the wavelength range bluer than $3700 \AA$ is of limited use, due to the sharp drop of the transmission of the PPAK IFU fore-optics and fibers in this wavelength range.

The exposure time is fixed for all targeted objects. For the V500 grating a single exposure of $900 \mathrm{~s}$ per pointing of the dithering pattern is taken, while for the V1200 grating 3 exposures of $600 \mathrm{~s}$ each are obtained per position. The exposure times were selected based on our previous experience with the instrument (in particular Marmol-Queralto et al. 2011, a feasibility study of the current survey), and expectations about the performance of the V500 grating (which was unknown at the beginning of the survey).

Data presented here were obtained during 16 nights (6 dark and 10 grey) in June-July 2010. A total of 20 objects were observed in both setups ( 1 more was observed only with the V500 grating). Table 1 lists the observed objects, including their coordinates, redshift, SDSS $g$-band observed and absolute magnitudes and $u-g$ color and their morphological classification (extracted from the NASA/IPAC Extragalactic Database ${ }^{4}$ ). These objects are described here since we will use them to illustrate the current status of the data acquisition and its quality.

\footnotetext{
${ }^{4}$ http://nedwww.ipac. caltech.edu/
} 
S.F. Sánchez et al.: CALIFA, the Calar Alto Legacy Integral Field Area survey. I.

Table 1. Summary of the objects observed in the first CALIFA runs.

\begin{tabular}{lccrrrrl}
\hline \hline Name $^{1}$ & RA $(\mathrm{J} 2000)^{2}$ & Dec $(\mathrm{J} 2000)^{2}$ & Redshift $^{2}$ & $g_{\text {SDSS }^{2}}$ & $M_{g}$ & $u-g^{2}$ & Morph. ${ }^{1}$ \\
\hline NGC 5947 & $15: 30: 36.00$ & $+42: 43: 01.00$ & 0.01965 & 14.20 & -20.41 & 1.57 & SBbc \\
UGC 09892 & $15: 32: 51.94$ & $+41: 11: 29.27$ & 0.01893 & 14.83 & -19.70 & 1.66 & Sb \\
UGC 10693 & $17: 04: 53.01$ & $+41: 51: 55.76$ & 0.02799 & 13.59 & -21.79 & 2.14 & $\mathrm{E}$ \\
UGC 10710 & $17: 06: 52.52$ & $+43: 07: 19.96$ & 0.02795 & 14.52 & -20.86 & 1.98 & Sb \\
NGC 6394 & $17: 30: 21.42$ & $+59: 38: 23.62$ & 0.02842 & 14.60 & -20.81 & 1.82 & SBb \\
NGC 6411 & $17: 35: 32.84$ & $+60: 48: 48.26$ & 0.01227 & 12.99 & -20.59 & 2.37 & E \\
NGC 6497 & $17: 51: 17.96$ & $+59: 28: 15.15$ & 0.02055 & 13.80 & -20.88 & 2.39 & SB(r)b \\
NGC 6515 & $17: 57: 25.19$ & $+50: 43: 41.24$ & 0.02284 & 13.72 & -21.22 & 2.03 & E \\
UGC 11228 & $18: 24: 46.26$ & $+41: 29: 33.84$ & 0.01935 & 14.04 & -20.54 & 2.03 & SB0 \\
UGC 11262 & $18: 30: 35.69$ & $+42: 41: 33.70$ & 0.01862 & 15.16 & -19.33 & 1.82 & Sd \\
NGC 6762 & $19: 05: 37.09$ & $+63: 56: 02.79$ & 0.00976 & 14.08 & -19.00 & 1.82 & S0/a \\
UGC 11649 & $20: 55: 27.62$ & $-01: 13: 30.87$ & 0.01265 & 14.01 & -19.64 & 2.29 & SB(r)a \\
UGC 11680* & $21: 07: 41.33$ & $+03: 52: 17.80$ & 0.02599 & 14.56 & -20.66 & 2.89 & Sb \\
NGC 7025 & $21: 07: 47.33$ & $+16: 20: 09.22$ & 0.01657 & 13.17 & -21.07 & 2.28 & Sa \\
UGC 11694 & $21: 11: 52.02$ & $+11: 16: 34.11$ & 0.01699 & 14.09 & -20.21 & 2.18 & S0 \\
UGC 11717 & $21: 18: 35.41$ & $+19: 43: 07.39$ & 0.02116 & 14.68 & -20.09 & 2.29 & S \\
UGC 11740 & $21: 26: 14.35$ & $+09: 47: 52.45$ & 0.02151 & 14.70 & -20.11 & 1.90 & S? \\
NGC 7194 & $22: 03: 30.93$ & $+12: 38: 12.41$ & 0.02718 & 13.87 & -21.44 & 2.02 & E \\
UGC 12127 & $22: 38: 29.41$ & $+35: 19: 46.89$ & 0.02745 & 13.88 & -21.46 & 2.56 & E \\
UGC 12185 & $22: 47: 25.06$ & $+31: 22: 24.67$ & 0.02217 & 14.35 & -20.52 & 2.03 & SBab \\
NGC 7549 & $23: 15: 17.26$ & $+19: 02: 30.43$ & 0.01573 & 14.14 & -19.98 & 2.01 & Sb? \\
\hline
\end{tabular}

Notes. ${ }^{(*)}$ This object has accurate data with the V500 setup only. ${ }^{(1)}$ Data obtained from the NED. ${ }^{(2)}$ Data obtained from the NYU-SDSS catalogue.

\section{Data reduction}

The reduction of the CALIFA data is performed using a fully automatic pipeline developed ad hoc. This pipeline operates without human intervention, producing both the scientifically useful frames and a set of quality control measurements that help to estimate the accuracy of the reduced data. The pipeline uses the routines included in the R3D package (Sánchez 2006a) and the E3D visualization tool (Sánchez et al. 2004), following the standard steps for fiber-based IFS (e.g., Sánchez 2006a; Sandin et al. 2010). The pipeline will be upgraded and improved during this project on the basis of the quality tests performed on the acquired data. Here we present a summary of the current implementation of the data reduction.

\subsection{Removal of electronic signatures and realignment of the frames}

The CCD used for CALIFA is a new $4 \mathrm{k} \times 4 \mathrm{k}$ E2 $\mathrm{V}$ detector installed in the instrument in October 2009. In contrast to the old $2 \mathrm{k} \times 4 \mathrm{k}$ one (Roth et al. 2005), the read-out software for the new CCD stores each frame in four different FITS files, corresponding to each of the four amplifiers of the detector, each one with different bias and gain levels. As a first step, the four files are combined into a single frame, after subtracting the corresponding bias level, and transforming from counts to electrons on the basis of the corresponding gain. A master bias frame is created by averaging all bias frames observed during the night, in order to cross-check the stability of the bias level. In contrast to the old $\mathrm{CCD}$, the new CCD does not exhibit any detectable structure in the bias frame.

The next step is clipping cosmic rays and combining different exposures taken at the same position on the sky. However, prior to combination, possible offsets between the projected spectra in the CCD due to flexure are estimated. As shown in Kelz et al. (2006), flexure is a major issue when working with PPAK. In general, the amplitude of the flexure is stronger while targets are setting. However, in a large survey like CALIFA, it will not be feasible to avoid observing setting targets $100 \%$ of the time. On the other hand, calibration frames are taken within a maximal range of $1.5 \mathrm{~h}$ from the science frames. This ensures that in most cases the flexure pattern of the calibration and science frames is the same and, therefore, it is possible to trace the spectra projected in the CCD and extract them properly. However, in a few cases (e.g. when the targets are observed when setting), there could be small linear offsets between the science and the calibration frames. To guarantee a correct handling of flexure, these offsets are estimated based on the position of several $(\sim 50)$ spots in the raw data. They correspond to the $\mathrm{Hg}$ emission lines of the arc lamps on one calibration frame, and the night-sky light pollution $\mathrm{Hg}$ emission (Sánchez et al. 2007a) on the science frames (including $\mathrm{Hg} \lambda 4047, \mathrm{Hg} \lambda 4358$ and $\mathrm{Hg}$ 15461). A linear shift is applied to the frame, when a significant offset $(>0.1$ pixel) is detected among the different exposures, and between them and their corresponding calibration frames (continuum and arc lamp exposures). This was the case in $\sim 2 \%$ of the frames taken during the first observing runs. Finally, a cosmic-ray cleaning algorithm is applied if only one single frame is taken at a certain position. The actual cleaning algorithm is based on the Laplacian Edge detection algorithm described in van Dokkum (2001), recently implemented in R3D.

\subsection{Spectral extraction, wavelength calibration and fiber transmission correction}

The locations of the spectra on the CCD are determined using a continuum-illuminated exposure taken before the science exposures. Each spectrum is then extracted from the science frames. In order to reduce the effects of cross-talk we do not perform a simple aperture extraction, which would consist of co-adding the flux within a certain number of pixels of the location derived from the continuum-illuminated exposure. Rather, we adopt a modified version of the Gaussian suppression (Sánchez 2006a), first used in the reduction of the PINGS data 
(Rosales-Ortega et al. 2010), and described in Sánchez et al. (2011). The cross-talk is reduced to less than $1 \%$ when adopting this new method. This procedure relies on an accurate internal focus of the instrument. The quality of the instrumental focus, which depends on the internal temperature of the structure of the instrument, is controlled by fitting a Gaussian to a subset of the projected continuum illuminated spectra along the cross-dispersion axis, and comparing the results with the nominal value for the instrument ( 2.5 pixels).

The extracted flux, for each pixel in the dispersion direction, is stored in a row-stacked-spectrum file (Sánchez et al. 2004). Wavelength calibration is performed using $\mathrm{HeHgCd}$ lamp exposures obtained before and after each pointing. The number of identified emission lines used to perform the wavelength calibration ranges between 11 (for the V1200 grating) and 16 (for the V500 grating), homogeneously distributed along the wavelength range covered. It was found that the adopted procedure yields an accuracy of about ten percent of the nominal pixel scale in the wavelength calibration (i.e., $\sim 0.2 \AA$ for the V500 grating and $\sim 0.1 \AA$ for the V1200 one). Differences in the relative fiberto-fiber transmission throughput are corrected by comparing the wavelength-calibrated row-stacked-spectra science frames with the corresponding frames derived from sky exposures taken during the twilight. These frames are also used to determine which pixels at the edge of the CCD are affected by the previously described vignetting effect. Those pixels for which the transmission drops to $<75 \%$ are masked.

\subsection{Sky subtraction}

PPAK is equipped with 36 fibers to sample the sky, distributed around the science fiber-bundle, in six small bundles of six fibers each, at a distance of $\sim 75^{\prime \prime}$ from the center of the FoV (see Kelz et al. 2006). By construction, the objects selected for the CALIFA final sample cover a substantial fraction of the FoV of the central PPAK bundle, with most of the sky fibers free from emission by the corresponding target. The procedure adopted to derive the night-sky spectrum is to combine the spectra corresponding to these fibers, performing a $2 \sigma$ clipping rejection to remove any possible contamination from low surface brightness regions of the galaxy and/or projected companions. Once obtained, the sky spectrum is subtracted from all spectra of the corresponding frame. The accuracy of the subtraction is controlled by comparing the equivalent width of the most prominent night-sky emission lines (Sánchez et al. 2007a), before and after the subtraction for each individual spectrum within the frame. In addition, each derived night-sky spectrum is used to derive the night-sky brightness at the location and time when the target is observed, by convolving the spectrum with the transmission curves of the Johnson $V$-band ( $B$-band) filter for the V500 (V1200) setups. This value is used to control the actual conditions when the data are acquired.

\subsection{Flux calibration}

Flux calibration is performed by comparing the extracted spectra of spectrophotometric standard stars from the Oke Catalogue (Oke 1990), observed during each night, with their corresponding flux-calibrated spectra, publicly available on the webpage of the observatory ${ }^{5}$. For the currently observed nights, the stars were: BD+25d4655, BD+28d4211 and BD+33d2642 (although any suitable star from the list will be used in the course of the

\footnotetext{
$\overline{5}$ http://www.caha.es/pedraz/SSS/sss.html
}

survey). When feasible, two different calibration stars are observed in the same night. Stars are not observed using the dithering pattern. A finite aperture of $r<10^{\prime \prime}$ was selected after several experiments, trying to maximize the covered flux and the signal-to-noise. This finite aperture, and the incomplete coverage of the FoV by the PPAK fiber bundle, produce a systematic offset of $15 \%$ in the flux calibration, estimated on the basis of simulated extractions of stellar images with a typical seeing of $\sim 1^{\prime \prime}$. This offset is well known, and it has already been reported by other authors (e.g. PINGS, Rosales-Ortega et al. 2010). This systematic offset has been corrected prior to any further analysis.

$\mathrm{R} 3 \mathrm{D}$ includes a procedure that finally provides the transformation function from observed counts to intensity, taking into account also the airmass and extinction of the observations of both the spectrophotometric standard stars and the science targets. To apply this procedure we adopted the extinction measured by the Calar Alto Visual EXtinction monitor (CAVEX) at the moment of the observations, and the average extinction curve for the observatory (Sánchez et al. 2007a). The procedure ensures a good relative flux calibration from the blue to the red part of the spectra, if the weather conditions throughout the night are stable. However, an absolute offset between the derived and real fluxes is expected if the weather conditions varied during the observations. If more than one standard star is observed, the pipeline performs a comparison between the transformations derived using each of them, in order to estimate the photometric stability of the night considered.

Figure 5 shows the derived transformation function from counts to flux density for both the V1200 and V500 gratings (corrected for the offset in resolution), together with those for the most frequently used gratings for PMAS (the V300 and V600 ones). These transformation functions were derived by selecting the curves for the most transparent nights included in the current study, together with the ones derived in a previous study (Marmol-Queralto et al. 2011, for the V300 and V600 gratings). It should be noted that there was a major update in the instrument, with an exchange of the CCD, between the observations taken with the V300 grating and the other observations. The offset between the efficiency found for this grating and the remaining gratings can easily be explained by the improvement in efficiency of the new CCD. Otherwise all efficiency curves are quite similar. This basic conclusion would not change if instead of using the best curve, we had selected the mean or median transformation function. These results are in agreement those reported by Roth et al. (2005), where it was stated that all the V-gratings show basically the same efficiency (after correcting for the resolution effects).

Figure 6 shows the dispersion between the different response curves found for different calibration stars and/or nights as a function of the wavelength for the V500 and V1200 gratings, normalized by the offset in the zeropoints at $4600 \AA$ and $4150 \AA$, respectively. The differences between one response curve and another as a function of the wavelength reflect the intrinsic dispersion of the flux calibration. The flux calibration obtained by applying this response curve is just a relative one. An absolute calibration is obtained by rescaling using a factor derived after the comparison with broad-band imaging, as described in forthcoming sections. On average, the relative flux calibration shows a dispersion of $\sim 2-3 \%$ ( $\sim 0.025 \mathrm{mag})$, for wavelengths redder than of $3850 \AA$. At shorter wavelengths, the error in the accuracy of the relative flux calibration becomes worse, being about $\sim 8 \%(\sim 0.08 \mathrm{mag})$ at $\sim 3700-3750 \AA$. These values are similar to the ones found in classical slit spectroscopy. 
S.F. Sánchez et al.: CALIFA, the Calar Alto Legacy Integral Field Area survey. I.
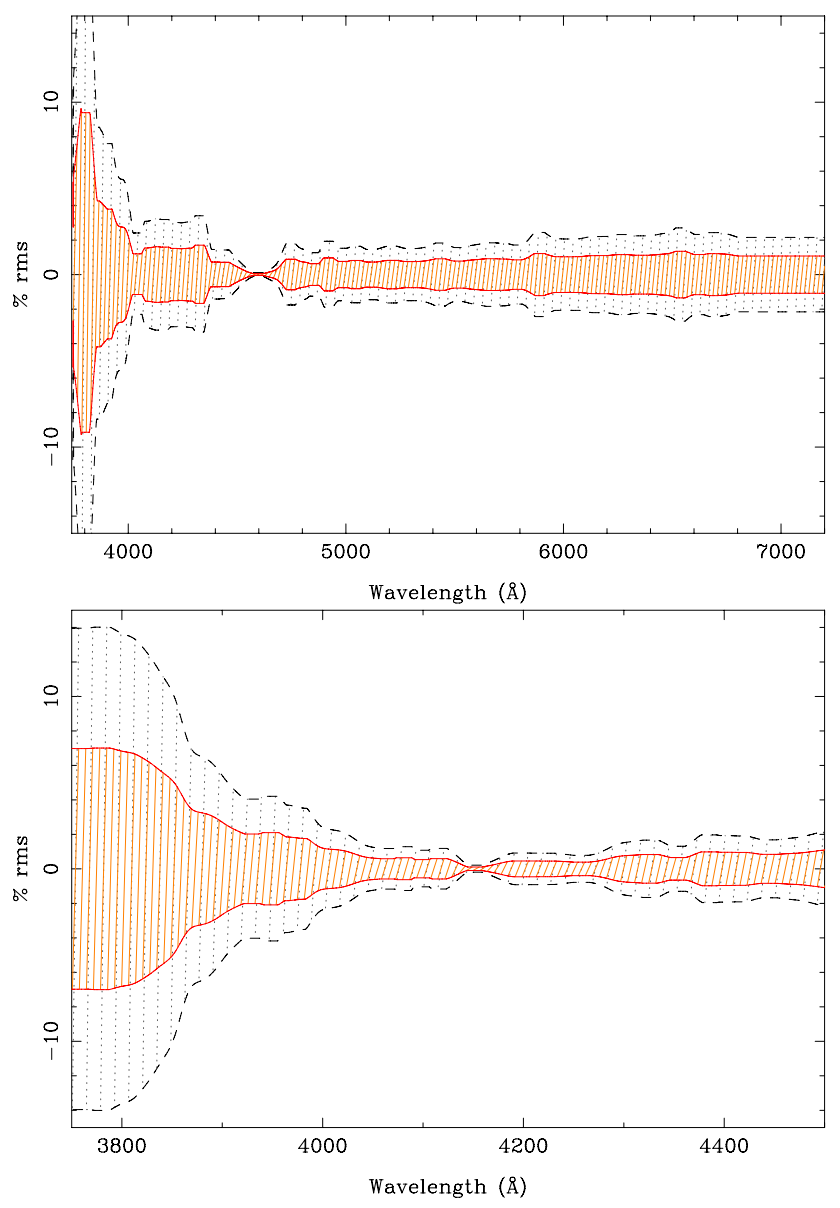

Fig. 6. Relative differences, in percent, between the response curves found on different nights for the V500 grating dataset (top panel) and the V1200 grating (bottom panel), as a function of wavelength, normalized to unity at $4600 \AA$ and $4150 \AA$, respectively. The solid hashed region (and red solid line) shows the $1 \sigma$ error, while the dashed hashed region (and black dashed line) shows the $2 \sigma$ error.

\subsection{Spatial re-arranging and image reconstruction}

Once the frames corresponding to each particular pointing are reduced, the science spectra corresponding to the three dithered exposures are combined in a single frame of 993 spectra. To do so the images are rescaled to a common intensity and response function by comparing the integrated spectra within an aperture of $30^{\prime \prime} /$ diameter. This procedure takes into account possible variations in the atmospheric transmission during the science exposures. In addition, the procedure produces a final position table, where the individual position tables (corresponding to the PPAK central bundle) are combined using the offsets provided for the dither. Finally, the data are spatially resampled to a datacube with a regular grid. This is done by adopting a fluxconserving variation of Shepard's interpolation method (Shepard 1968). This method is a very simple and robust tessellation-free interpolation of scattered data, where the intensity at each interpolated point is the sum of a weighted average of the intensities corresponding to $n$ adjacent scattered points (the original spectra) within a certain boundary distance $\left(r_{\mathrm{lim}}\right)$ :

$$
F(i, j)=\sum_{k=1}^{k=n} w_{i, j}^{k} f_{k} \quad r_{1 \ldots n}<r_{\mathrm{lim}}
$$

where $F(i, j)$ is the reconstructed intensity in the pixel $(i, j)$ of the final datacube at a certain wavelength, $w_{i, j}^{k}$ is the weight at this pixel of the adjacent spectrum $k$, and $f_{k}$ is the intensity of this adjacent spectrum, at the considered wavelength.

The weights are derived by a Gaussian function:

$w=N \exp \left[-0.5(r / \sigma)^{2}\right]$

where $N$ is a normalization parameter, $r$ is the distance between the pixel $(i, j)$ and the spectrum $k$, and $\sigma$ is the parameter that defines the width of the Gaussian function. The normalization parameter is derived for each interpolated pixel, being defined as the inverse of the sum of the different weights of the spectra contributing to this pixel:

$N(i, j)=\frac{1}{\sum_{k=1}^{k=n} w_{i, j}^{k}} \quad r_{1 \ldots n}<r_{\text {lim }}$.

This normalization guarantees that the integrated flux is preserved. The adopted image reconstruction assumes a boundary limit of $r_{\text {lim }}=5^{\prime \prime}, \sigma=1^{\prime \prime}$, and a final pixel scale of $1^{\prime \prime} /$ pixel for the resulting datacube. Prior to this interpolation, the flux corresponding to those spectral pixels masked due to the vignetting are replaced by the average of the fluxes at the two nearest fibers not affected by this effect. Finally, the flux intensity is corrected by the difference in aperture between the original fibers $\left(\sim 2.7^{\prime \prime} /\right.$ diameter $)$ and the final pixels.

\subsection{Differential atmospheric refraction}

The differential atmospheric refraction (DAR, Filippenko 1982) is corrected once the data are spatially resampled to a datacube with a regular grid. In the case of IFS data, the DAR can be corrected empirically, after the observations, without requiring to know the original orientation of the instrument and without the need of a compensator (Emsellem et al. 1996; Arribas et al. 1999; Roth et al. 2004). To do so, the reconstructed datacubes are thought of as a set of narrow-band images with a band-width equal to the spectral resolution. These images can be recentered using the theoretical offset determined by the DAR formulae (Filippenko 1982), or, as in 2D imaging, tracing the intensity peak of a reference object in the field-of-view (or a DAR reference observation) along the spectral range, and recentering it. Note that this latter approach is basically unfeasible in slit spectroscopy, this being one of the fundamental differences between the two methods.

We adopted this latter empirical correction for the CALIFA pipeline, using the corresponding tools included in R3D. For doing so, the centroid of the observed object (i.e., the galaxy) is derived at each wavelength, by determining its barycenter in an image slice extracted from the datacube, within a range of $20 \AA$ around the considered wavelength. Then, the shifts along the wavelength are determined by comparing the corresponding coordinates to a common reference. A polynomial function of order 3 is fitted to each shift $(X$ and $Y$ ) along the wavelength to increase the accuracy of the determined offset (e.g. Wisotzki et al. 2003). Then, the full datacube is shifted by resampling and shifting each image slice at each wavelength. In general, the empirical correction produces similar results to the theoretical one. However, the theoretical correction is not well defined when different pointings of the same object are observed during different nights, due to weather restrictions $(\sim 15 \%$ of the observed targets). These observations may span over different dark-time periods. The use of the empirical correction guarantees the homogeneity of the treatment of DAR correction for all the datasets. 

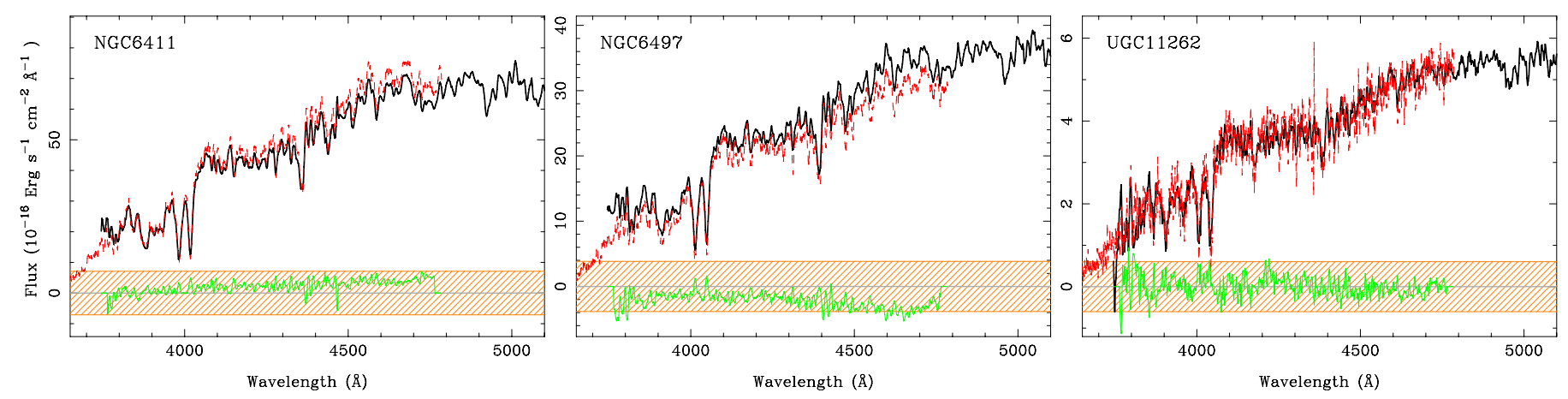

Fig. 7. Comparison of the central 5" spectrum extracted from the V500 (black, thick solid line) and V1200 (red, dashed line) grating datacubes for three typical objects (from left to right): NGC 6411, the brightest object observed so far; NGC 6497, a spiral galaxy with an average brightness, and UGC 11262, the faintest target observed so far. The green thin solid line shows the difference between both spectra. The orange hashed region indicate the $\pm 10 \%$ median intensity range of both spectra around the zero level, indicated with a grey continuous line.

\subsection{Absolute flux re-calibration}

In order to get the best possible absolute flux calibration, we recalibrate our data based on SDSS photometry, available for all targets by construction of the sample. Of the five SDSS filters (ugriz), two namely, the $g\left(\lambda_{\mathrm{eff}}=4770 \AA\right)$ and $r\left(\lambda_{\mathrm{eff}}=6231 \AA\right)$ filters, are covered almost completely by the V500 grating data, while $u$ ( $\lambda_{\text {eff }}=3594 \AA$ ) is only partially covered by both the V500 and V1200 grating data. Therefore, we use the V500 grating data and the $g$ and $r$ band SDSS images to perform a primary flux recalibration.

To perform the primary recalibration, we measure the counts of each galaxy in the SDSS image inside a $30^{\prime \prime}$ diameter aperture. These counts are converted to flux density following the counts-to-magnitude prescription in the SDSS documentation ${ }^{6}$. The accuracy of this photometry was cross-checked by obtaining the magnitudes of any detected star in each field, using the same procedure, and comparing them with the values listed in the SDSS DR6 photometric catalogue. We obtained less than 0.05 magnitude dispersion for the stars with $g<17.5$ mag. Once we ensure the accuracy of this photometry, we extract the spectrophotometry from the reduced datacubes corresponding to the V500 grating setup, coadding the flux of individual spectra inside a $30^{\prime \prime}$ diameter aperture and convolving this spectrum with the SDSS $g$ and $r$ filter passbands (ADPS database, Moro \& Munari 2000). Using these two data pairs, a scaling solution is found, by adopting the average of the flux ratio in both bands.

Once the V500 datacube has been recalibrated, a new scaling solution for the V1200 data is derived by comparing the $5 "$ aperture extracted spectra around the central position of each object from these cubes with those extracted from the original reduced V1200 data. In this case, a low-order polynomical function is fitted to the ratio between both spectra within the common wavelength range $(\sim 3745-4770 \AA)$. The result of this polynomial fitting is then applied to the V1200 data, to match them to the V500 ones. Figure 7 shows the central aperture extracted spectra for both setups together with the difference between them ( $5^{\prime \prime} /$ diameter $)$, for three typical targets. It illustrates the agreement between both datasets, once the recalibration procedure has been applied. The V1200 spectra have been convolved with a Gaussian function to compensate for the difference in resolution, for the purpose of this figure only. The typical difference between the spectra is $\sim 10 \%$ of the average. The strongest differences are located at the wavelengths of the

\footnotetext{
${ }^{6}$ http://www.sdss.org/dr6/algorithms/fluxcal.html
}

more significant spectral features (absorption and/or emission lines).

We conclude that the current version of the pipeline, which operates automatically, is able to reduce the data, producing the required data cubes to perform early experiments and estimations of their quality. The pipeline will be continuously upgraded, producing different versions of the data, on the basis of the results of the different foreseen quality controls, prior to releasing the data to the community. In the next section we describe the early tests implemented to estimate the quality of the data. In some cases these tests have already induced slight modifications in the pipeline, as we will describe below in detail.

\section{Quality of the data}

In parallel to the reduction of the data, the pipeline performs a set of automatic tests, which are stored during the reduction process, creating a set of tables and figures. These allow the CALIFA team to check the quality of the data and to identify possible problems in the data reduction. In this section we present the basic results of the currently implemented quality controls on the data acquired so far. We expect the quality control to become more comprehensive with time.

\subsection{Accuracy of the wavelength calibration}

In general, the wavelength solution is found with an accuracy of the order of $10-15 \%$ of the nominal pixel scale, i.e., the rms of the fit with the low order polynomial function is $\sim 0.2-0.3 \AA$ for the V500 grating and $\sim 0.1-0.2 \AA$ for the V1200 grating.

In order to obtain an independent estimate of the accuracy of the wavelength calibration of the science frames, we compare the nominal and recovered wavelengths of the most prominent night-sky emission lines in each individual spectrum prior to the sky subtraction. The central wavelength of these lines was determined by fitting a Gaussian function to each line. This provides us with 331 estimations of the relative offsets between both values for each considered night-sky line. For the V500 grating we use the $\mathrm{HgI} \lambda 5461$, [OI] $\lambda 5577$, NaD $\lambda 5893$ and [OI] $\lambda 6300$ lines, while for the V1200 grating we use the $\mathrm{HgI} \lambda 4046$ and $\mathrm{HgI} \lambda 4358$ ones. Only measurements derived using emission lines with a signal-to-noise higher than 10 are considered. Finally, a few hundred measurements of the differences between the nominal and recovered wavelengths are retained for 
S.F. Sánchez et al.: CALIFA, the Calar Alto Legacy Integral Field Area survey. I.

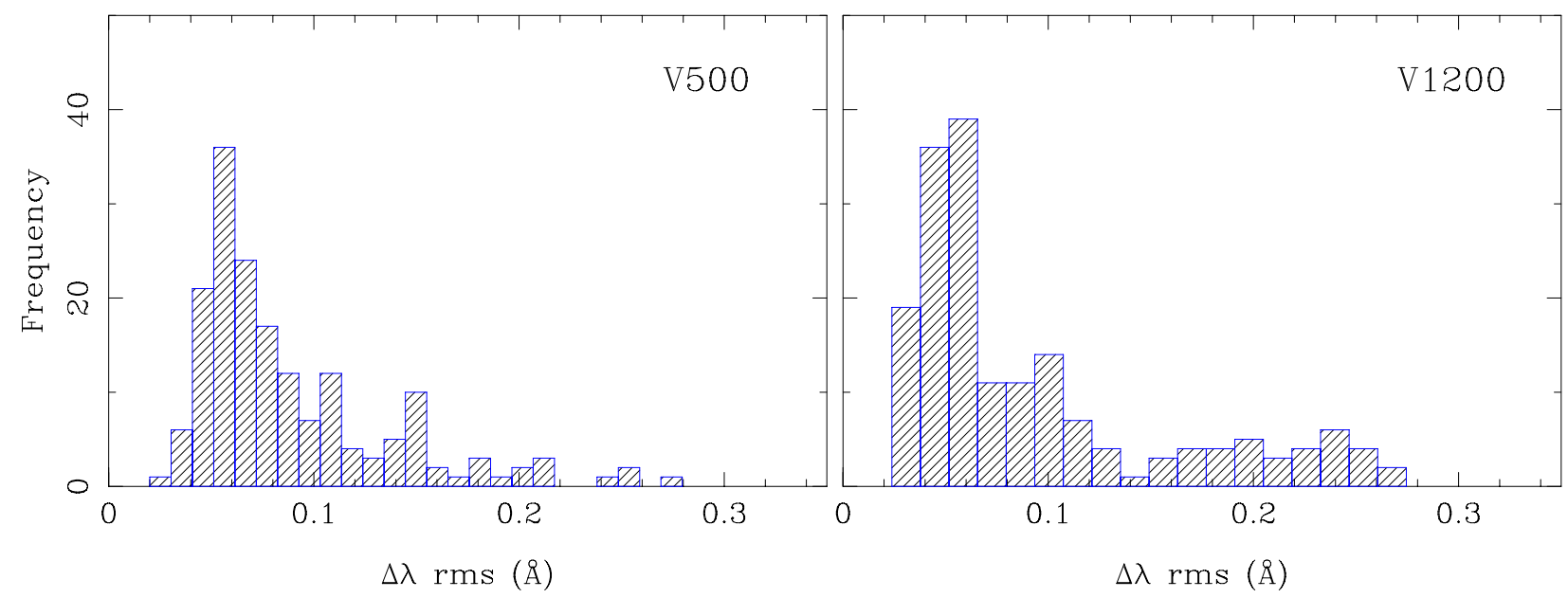

Fig. 8. Distribution of the rms of the difference between the nominal and measured wavelengths of the night-sky emission lines, derived from the statistical analysis of all the considered emission lines within the same science frame for the V500 (left panel) and V1200 (right panel) grating datasets. The mean shift is around zero.

each frame corresponding to both gratings. A simple statistical analysis allows us to estimate the final accuracy of the wavelength calibration, deriving both the median offset (if any) and the rms of each dataset.

No mean shift was found between the nominal and measured wavelength for the different analyzed sky lines. Figure 8 shows the distribution of the rms of the difference between the nominal and measured wavelengths of the night-sky emission lines, found for each science frame, for both setups (left and central panel). In both cases the values range between $0.01 \AA$ and $0.35 \AA$. The resulting median value is very similar for both distributions, being around $\sim 0.1 \AA$, which corresponds to $\sim 8 \mathrm{~km} \mathrm{~s}^{-1}$ at the wavelength of [OII] $\lambda 3727$ and $\sim 3 \mathrm{~km} \mathrm{~s}^{-1}$, at the wavelength of $\mathrm{H} \alpha$.

\subsection{Empirical spectral resolution}

The spectral resolution of a dataset is limited at best to the nominal instrumental resolution. However, different effects, such as problems with, or degradation in, the internal focus of the instrument, errors in the tracing/extraction process or in the wavelength calibration may affect the final spectral resolution.

In order to estimate the real spectral resolution for each science frame, we fit the strongest night-sky emission lines (see Sect. 6.1) in each individual science spectrum and before subtracting the night-sky spectrum with a Gaussian function using FIT3D (Sánchez et al. 2006b). Night-sky lines are unresolved at our resolution, thus their widths provide good estimates of the final resolution at their respective wavelengths. The median and standard deviation of the FWHMs are stored for each science frame.

The spectral resolution found for the V1200 grating is very similar to the nominal value $(\sim 2.7 \AA)$, and is consistent with the values reported by Roth et al. (2005). For the V500 grating on the other hand, there is no published information about its nominal spectral resolution, which was estimated to be $\sim 6 \AA$ (Roth, priv. comm.). The empirical resolution estimated from night-sky lines is $\sim 6.5 \AA$. These instrumental spectral resolutions correspond to velocity resolutions of $\sigma \sim 85 \mathrm{~km} \mathrm{~s}^{-1}$ and $\sigma \sim 150 \mathrm{~km} \mathrm{~s}^{-1}$ for the V1200 and V500 datasets, fulfilling the requirements of the survey, for both setups.
The same experiment can be repeated using the arc-lamp frames obtained during the night instead of the science frames. This procedure has the advantage that all selected lines have high signal-to-noise, and are evenly distributed over the full spectral range. However, the derived instrumental dispersion cannot be directly compared with the science data, since the arc-lamp exposures are an order of magnitude shorter in time and, therefore, they are not affected by the degradation in resolution due to the drift of the spectra on the CCD in long exposures (due to the change of the flexure pattern). For this experiment, a datacube for each reduced arc lamp frame was created following the prescriptions for science frames. Then, for each spectrum of this datacube, we fit each emission line with a single Gaussian function, deriving the central wavelength, intensity and width (in FWHMs). These latter values are considered as an estimate of the instrumental dispersion. As expected the derived values are smaller (and more precise) than thos reported above, being $2.30 \pm 0.11 \AA$ for the V1200 (ranging between $2.2-2.4 \AA$ ) and $5.65 \pm 0.21 \AA$ for the V500 (ranging between 5.3-6.1 $\AA$ ). However, the final resolution achieved in the data is more similar to that estimated from the night-sky lines, than that estimated from the ARC-lamps, which are observed in shorter integration times.

Although not clearly appreciated in the measurements performed using the night-sky lines, there is a clear spatial and wavelength dependence of the instrumental resolution. Figure 9 shows both distributions, for the V1200 data (a similar result is found for the V500 data). The left panel shows the distribution with wavelength, which shows the larger difference, i.e. $\sim 13 \%$. At each wavelength we plot values found at different locations within the FoV, shown in the right-panel. The amplitude of the spatial pattern is $\sim 5 \%$. The pattern seen in the spatial distribution, which has a maximum in an annular ring at $\sim 15^{\prime \prime}$ from the center, and an underlying gradient, is a consequence of the correspondence between the spatial distribution of the PPAK fibers within the FoV and their positions along the entrance pseudoslit of the spectrograph. The fibers located in the described ring correspond to those at the edges of the pseudo-slit. This implies they also lie at the edges of the CCD, where the resolution is worse. The underlying gradient is possibly an effect of a slight tilt of the focal plane of the CCD with respect to the focal plane of the spectrograph camera. 

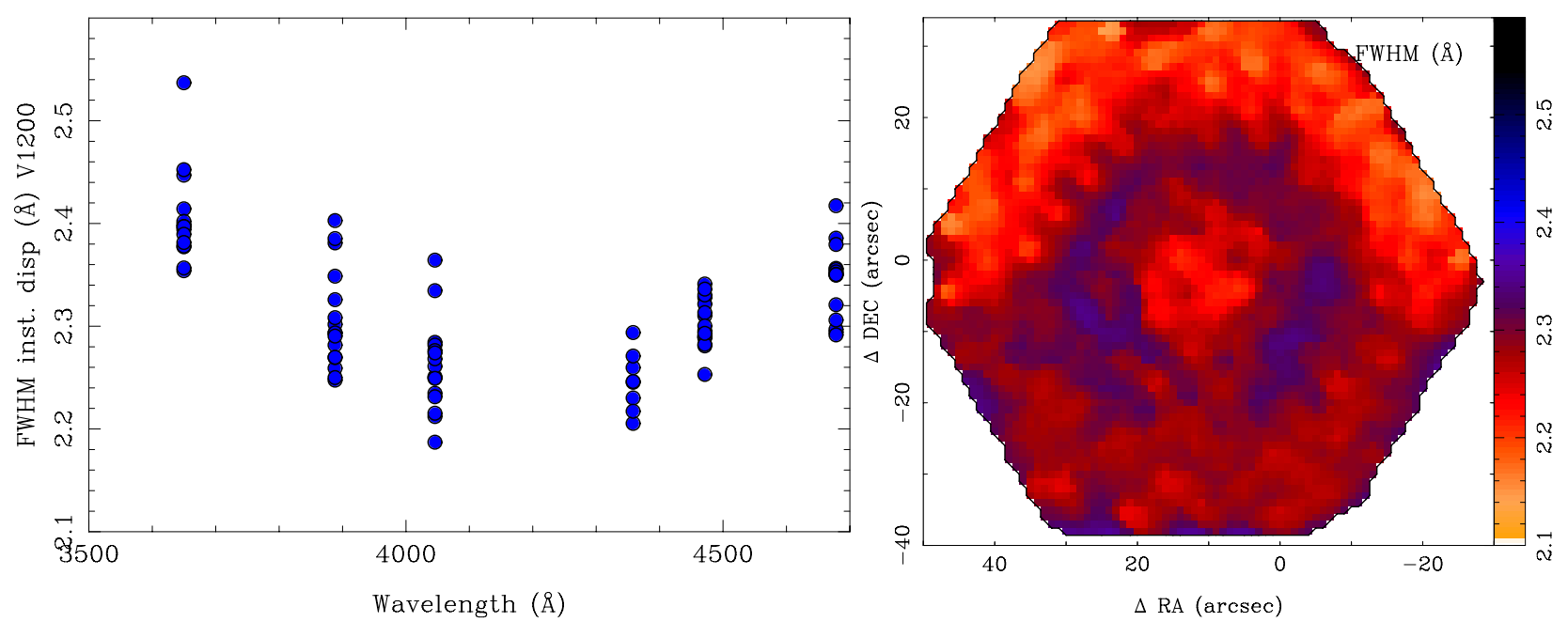

Fig. 9. Left panel: the distribution of the FWHM of the instrumental dispersion derived from the analysis of the emission lines within the arc-lamp frames at different wavelengths and different positions within the FoV, for the V1200 data. Right panel: the spatial distribution of the average FWHM of the instrumental dispersion, derived from the same arc lines.

The correction of these instrumental signatures were not originally considered in the version of the pipeline described in Sect. 5. These data allowed us to understand the changes in resolution along the wavelength and across the field. We found no clear dependencies of the instrumental resolution with the positioning of the telescope, at least for the range of airmasses at which we observe the targets $(<1.4)$. There is a clear dependency with the temperature of the instrument, which affects its internal focus. This is corrected by the focus procedure adopted every night, and a careful monitoring of this temperature (changes larger than $\sim 0.5^{\circ} \mathrm{C}$ are rare, along a single night). However, both effects produce mostly a change in the global value, rather than a change in the described pattern, which seems to be rather stable.

Based on these results, we implemented a procedure in the pipeline to correct for the differences in the spectral resolution across the FoV and along the spectral range by fixing it (in terms of FWHM) to the worst value found for each dataset (based on the measurements of the FWHM in the ARC-lamp frames). For doing so, the described derivation was repeated for each arclamp exposure, associated with each science frame. Then a low order polynomial function is fitted to the derived values of the resolution, per spaxel and per pixel. Finally, the differential resolution is derived by obtaining the quadratic difference between the estimated value at each pixel and the globally worst value. The science spectra are then convolved pixel-to-pixel by this differential resolution, normalizing it along the spectral range and across the FoV. The final resolution achieved is slightly worse than the one described above, due to flexure-related shifts in wavelength: $\sim 2.7 \AA$ for the V1200 and $\sim 6.5 \AA$ for the V500, as derived from the measurements of the night-sky emission lines width. However, with the adopted procedure the resolution is normalized along the spectral range and across the field.

Any further analysis described in this article is based on these spectra, with a homogeneous spectral resolution (in terms of FWHM).

\subsection{Accuracy of the sky subtraction}

The determination of the night-sky spectrum and its subtraction from the data is an issue in many IFUs, mostly due to the small FoV and the resulting lack of a pure night-sky spectrum. By design the CALIFA sample galaxies have an optical size that fits within the FoV of the central bundle of the PPAK IFU, while most of the 36 sky fibers are free of any contamination from galaxy light. The procedure adopted to subtract the sky is therefore rather simple, as explained above (Sect. 5.3).

In order to estimate the accuracy of this procedure, we measured the flux corresponding to the strongest night-sky lines before and after subtracting the estimated night-sky spectrum. These lines are [OI] $\lambda 5577 \AA$ for the V500 grating and $\operatorname{Hg} \lambda 4358 \AA$ for the V1200 grating. Their typical intensity ranges between $1-6 \times 10^{-17} \mathrm{erg} \mathrm{s}^{-1} \mathrm{~cm}^{-2}$, measured from adding the flux within a range of 15 pixels around their nominal wavelengths, and subtracting the flux in two adjacent continua of the same width, at shorter and longer wavelengths. After subtracting the night-sky spectra the typical residual value ranges between $\pm 0.5 \times 10^{-16} \mathrm{erg} \mathrm{s}^{-1} \mathrm{~cm}^{-2}$ for $99 \%$ of the spectra, i.e., the sky is subtracted with an accuracy of 1-5\%, with a typical residual of less than $2 \%$. Figure 10 shows the distribution of intensities obtained for each considered line once the night-sky spectra have been subtracted.

The reduction pipeline stores the actual night-sky spectrum subtracted from each science frame, to allow inspection in more detail if needed. In particular, the night-sky surface brightness is measured by the pipeline and stored as a parameter to evaluate the quality of the data. To do so, each night-sky spectrum is convolved with the $B$-band (V1200) and $V$-band (V500) filter response curves obtained from the ADPS database (Moro \& Munari 2000), and then the corresponding surface brightness was estimated, applying the zero points listed in Fukugita et al. (1995). For the currently observed data the median values of the derived night-sky surface brightness are within $\sim 0.5 \mathrm{mag}$ of the typical values for a dark night at Calar Alto (Sánchez et al. 2007a).

\subsection{Accuracy of the flux calibration}

The selected observational and reduction strategy uses the spectrophotometric calibration stars observed during the nights to perform an initial flux calibration. The main aim of this flux calibration is to correct for the relative transmission of the system from blue to red, and provide a preliminary absolute zero point. 
S.F. Sánchez et al.: CALIFA, the Calar Alto Legacy Integral Field Area survey. I.
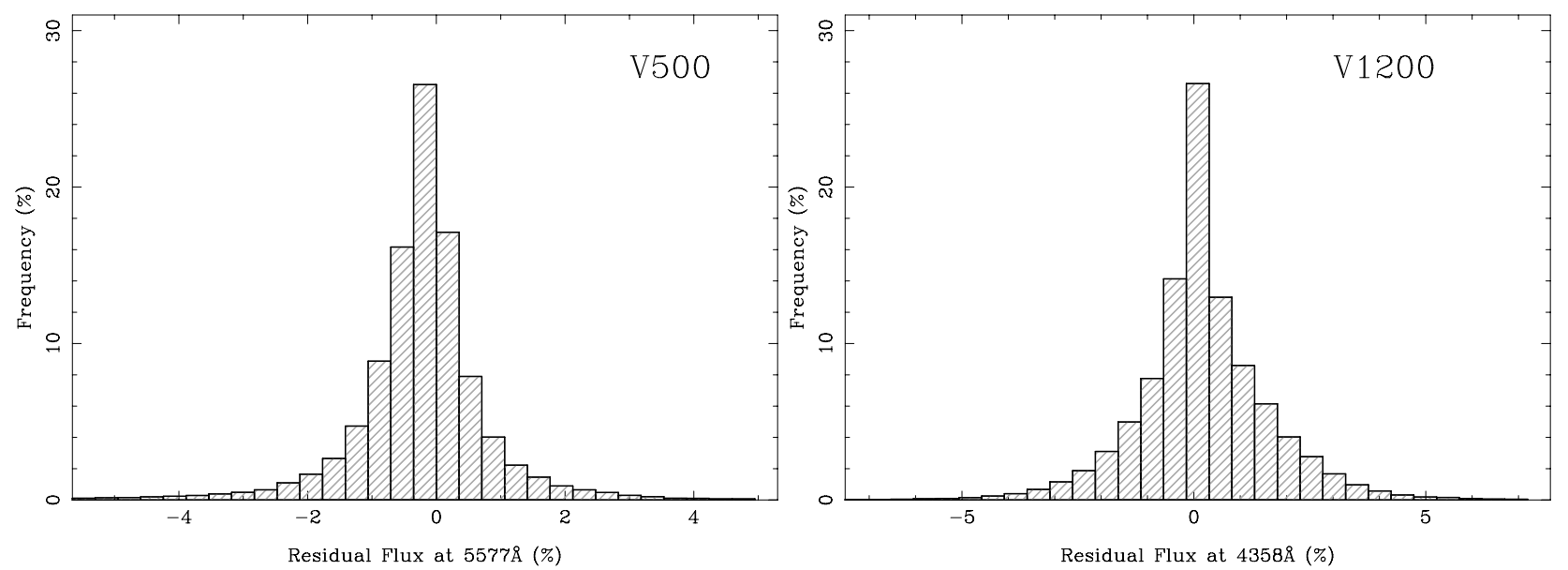

Fig. 10. Distribution of the relative residual flux after subtracting the night-sky spectrum for the strongest emission lines on the corresponding wavelength range covered by each setup: [OI] $\lambda 5577$ for the V500 grating (left panel) and HgL $\lambda 4358$ for the V1200 grating (right panel).

As indicated in Sect. 5, the accuracy of the relative transmission is better than a few percent in most of the covered wavelength range, which is sufficient for the science goals of this survey. On the other hand, the accuracy of the absolute spectrophotometric calibration was $\sim 24 \%$, on average, for the clear/photometric nights, and it can be worse for data taken under non-photometric conditions. Therefore a flux recalibration procedure is implemented in the pipeline, anchoring our photometry to that of the SDSS data (Sect. 5.7). It is expected that the final absolute spectrophotometric accuracy improves to $\sim 8 \%$ photometric error, on the basis of our previous experience with the PINGS data (Rosales-Ortega et al. 2010).

We cross-check the improvement in the spectrophotometric calibration by extracting from the datacubes the integrated spectra within an aperture of 30" diameter around the peak intensity of the object, before and after the flux recalibration. The corresponding $g$ and $r$-band magnitudes were derived from these spectra, following the procedures described in Sect. 5. The magnitudes derived from the spectroscopy were then compared with the corresponding ones obtained from the SDSS images, adopting a similar aperture, and following the same procedure as used to recalibrate the data.

Figure 11 shows the distribution of the $g$-band magnitudes derived from the datacubes and the SDSS images before (blue solid circles) and after (red solid squares) the recalibration procedure. As expected, there is a significant improvement in the photometric agreement. Prior to the recalibration, both photometric datasets match within $\sim 20 \%$. This dispersion is similar to that found in the zero points of the flux calibration derived night-by-night for the V500 data. Once the recalibration was applied, both datasets match each other within $\sim 0.06$ mag, i.e., $\sim 7 \%$ (Fig. 11, red squares). Similar results are found for the $r$-band.

To reinforce the validity of our flux re-calibration procedure, we repeated it for the flux-calibration standard stars observed during the run. The frames corresponding to the calibration stars were reduced using the same procedure as used for the science objects. Instead of using the transmission curve derived for the corresponding night, we adopted the master curve shown in Fig. 5, and the extinction information provided by the extinction monitor. With this procedure we try to simulate as closely as possible the reduction conditions of the science frames. Next, to apply the same re-calibration procedure outlined before, the

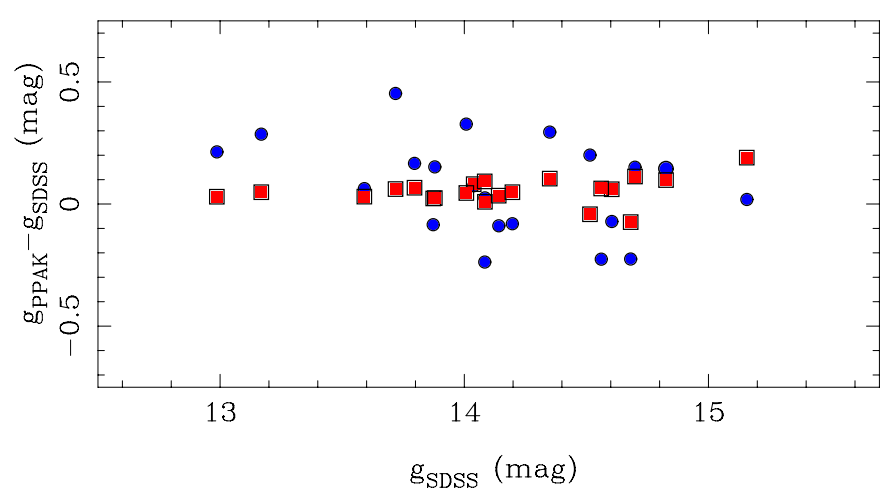

Fig. 11. Comparison of the $g$-band magnitude derived from the integrated spectrum within an aperture of $30^{\prime \prime}$ diameter extracted from the V500 datacubes and the corresponding magnitudes derived from the SDSS images, before (blue solid circles) and after (red solid squares) applying the flux recalibration procedure described in the text.

$g$ and $r$-band magnitudes were synthesized from the published flux-calibrated spectra. The top panel of Fig. 12 shows a typical example of how the flux-recalibrated spectrum corresponding to a calibration star agrees with the published flux-calibrated one. The bottom panel shows the distribution of the relative differences in intensity, pixel-to-pixel, between the published and re-calibrated spectra, for all the calibration stars observed along the run. The standard deviation is below $\sim 5 \%$ for the considered wavelength range (3800-7000 ̊)

\subsection{Signal-to-noise and depth of the data}

The pipeline performs a rough estimation of the $S / N$ in each individual spectrum within the final reduced datacube. The median and the standard deviation of the intensity values are computed over a wavelength range free from strong spectral features. The wavelength range selected to perform this analysis was 4480$4520 \AA$ for both gratings, in order to simplify the comparison between the derived values. Assuming that the scatter is entirely due to noise, which is a rather conservative assumption, the signal-to-noise per spatial pixel is estimated as the ratio between the standard deviation and the median flux. Figure 13 illustrates the result of this procedure for the V500 datacube of NGC 5947. 

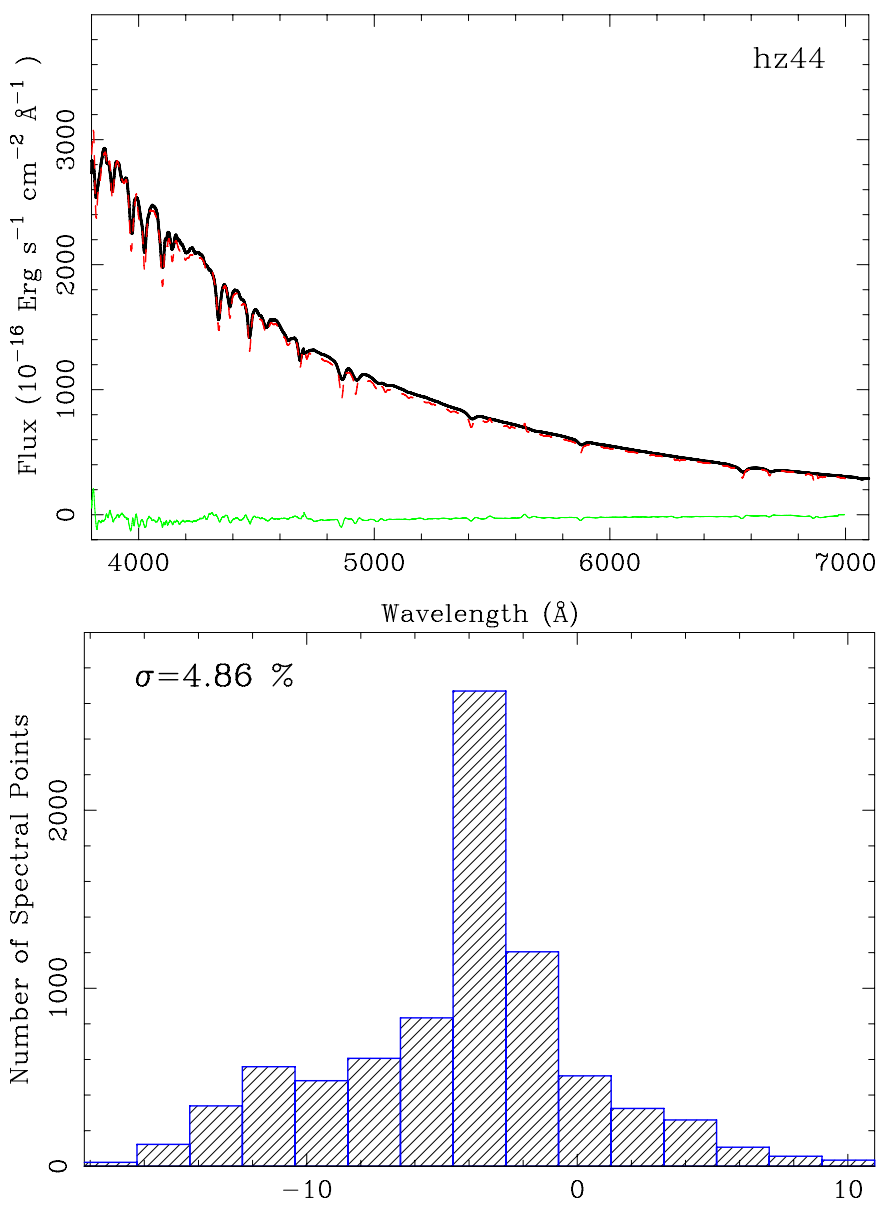

Relative Differences (in \%)

Fig. 12. Top panel: comparison of the published flux-calibrated spectrum of a calibration star (black, thick solid line), with the flux recalibrated spectrum extracted from the V500 data (red, dashed line). The green line shows the residuals, once corrected by the different resolution of each spectra. Bottom panel: distribution of the residuals in intensity, pixel-to-pixel, for all the calibration stars observed along the run. The standard deviation is lower than $\sim 5 \%$ for the considered wavelength range (3800-7000 ̊).

A typical patchy structure results for most of the objects, due to the intrinsic differences in the signal-to-noise map in dithered exposures.

Once the signal-to-noise map was obtained, we determined those pixels for which $S / N$ ranges between 3 and 4 . The average flux corresponding to these spaxels $(\sim 100-200$ spaxels $)$ is considered a rough estimation of the $3 \sigma$ detection limit of the considered datacube. This flux is transformed to the corresponding $\mathrm{AB}$ magnitude using the standard equations, and stored for further quality control. The depth of the datacubes depends on several factors, for each considered setup. It is mostly related to the night-sky brightness and the transparency and photometric stability of the night. Other factors, like the effects of the atmospheric seeing, have a much reduced effect due to the large size of the PPAK fibers $\left(\sim 2.7^{\prime \prime}\right.$ diameter $)$.

The datacubes reached a $3 \sigma$ signal-to-noise ratio for an average surface brightness of $\sim 23.0 \mathrm{mag} / \mathrm{arcsec}^{2}$ for the objects observed under good weather conditions with the V500 grating, and $\sim 22.7 \mathrm{mag} / \operatorname{arcsec}^{2}$ for those observed with the V1200 grating. This difference is expected since the difference in resolution between both setups is not completely compensated by the larger integration time for the latter grating. The area sampled with a

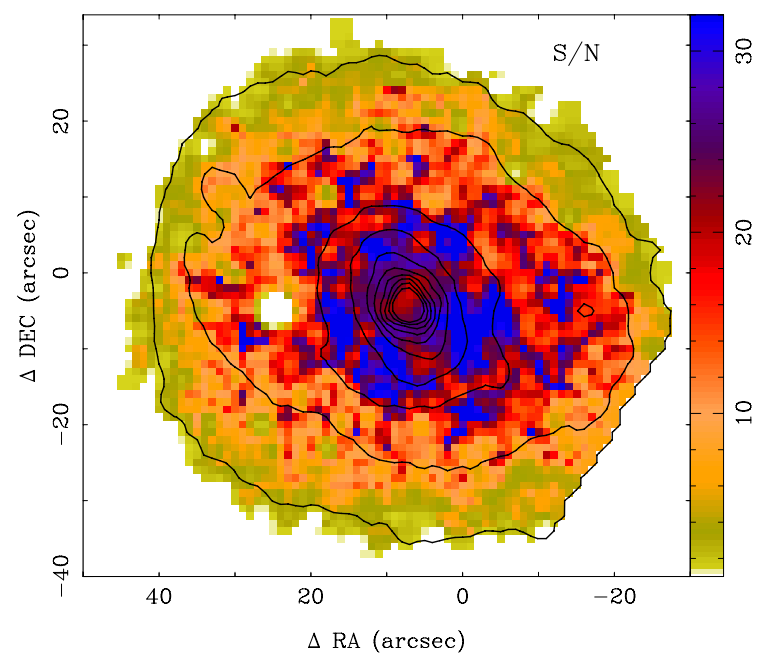

Fig. 13. Color image of the signal-to-noise map derived for the V500 data corresponding to the continuum emission of the NGC 5947 galaxy at the wavelength range between 4480 and $4520 \AA$, based on the rough estimation described in the text. The contours shows the intensity of the $V$-band image synthesized from the datacube, with the 1st contour at $1.5 \times 10^{-18} \mathrm{erg} \mathrm{s}^{-1} \mathrm{~cm}^{-2} \operatorname{arcsec}^{-2}\left(\sim 23.5 \mathrm{mag} / \operatorname{arcsec}^{2}\right)$ and consecutive ones with a step of $3 \times 10^{-18} \mathrm{erg} \mathrm{s}^{-1} \mathrm{~cm}^{-2} \operatorname{arcsec}^{-2}$.

$S / N$ above $3 \sigma$ is $\sim 0.7 \mathrm{arcmin}^{2}$, for the V500 grating. This is clearly identified in Fig. 13, where the contours indicate the intensity level in the $V$-band, extracted from the datacube. The first contour, at $\sim 3 \sigma$ corresponds to $\sim 23.5 \mathrm{mag} / \operatorname{arcsec}^{2}$ in this particular object. Both the limiting magnitude and the area sampled over the $3 \sigma$ limit agree with our expectations. The depth and area of the FoV sampled above the $3 \sigma$ limit are very similar for all the observed targets: the $3 \sigma$ surface brightness depth has a range of $\pm 0.2 \mathrm{mag}$ of the average values of each target and the area of the FoV above the $3 \sigma$ limiting surface-brightness ranges between a $50 \%$ and a $90 \%$. This is because, due to the diameter selection of the sample, all the targets have similar surface brightness at a similar projected distance independently of their real physical size or light distribution. In addition, as all the targets are observed in dark time, there is no expected variation of the $S / N$ or depth with night-sky brightness.

Obviously, this is only true for the objects observed on clear weather conditions. Under bad weather conditions the $S / N$ is degraded. There are two cases of bad weather conditions considered in the design of the survey: (1) completely lost nights (i.e., clouds, snow, rain...), and (2) nights with partial observations, i.e., nights with data taken under not-so-good weather conditions. For the first kind of nights, we have accounted an overhead of $\sim 30 \%$, based on published estimations of the fraction of useful time (Sánchez et al. 2007a), and they were considered in the total budget of nights. For the second case, our actual estimation is that only $\sim 10 \%$ of the targets are observed under sub-optimal conditions. Of those, $\sim 60 \%$ corresponds to the V1200 setup, due to the larger fraction of time devoted to these observations. In summary, it is expected that we will obtain good quality data (i.e., with the described $S / N$ and depth) for more than 500 galaxies, once the survey is completed.

\subsection{Comparison with SDSS data}

As indicated above, the CALIFA mother sample has been selected from the SDSS imaging survey. This selection provides us with a large ancillary dataset, including imaging in five bands for 
S.F. Sánchez et al.: CALIFA, the Calar Alto Legacy Integral Field Area survey. I.
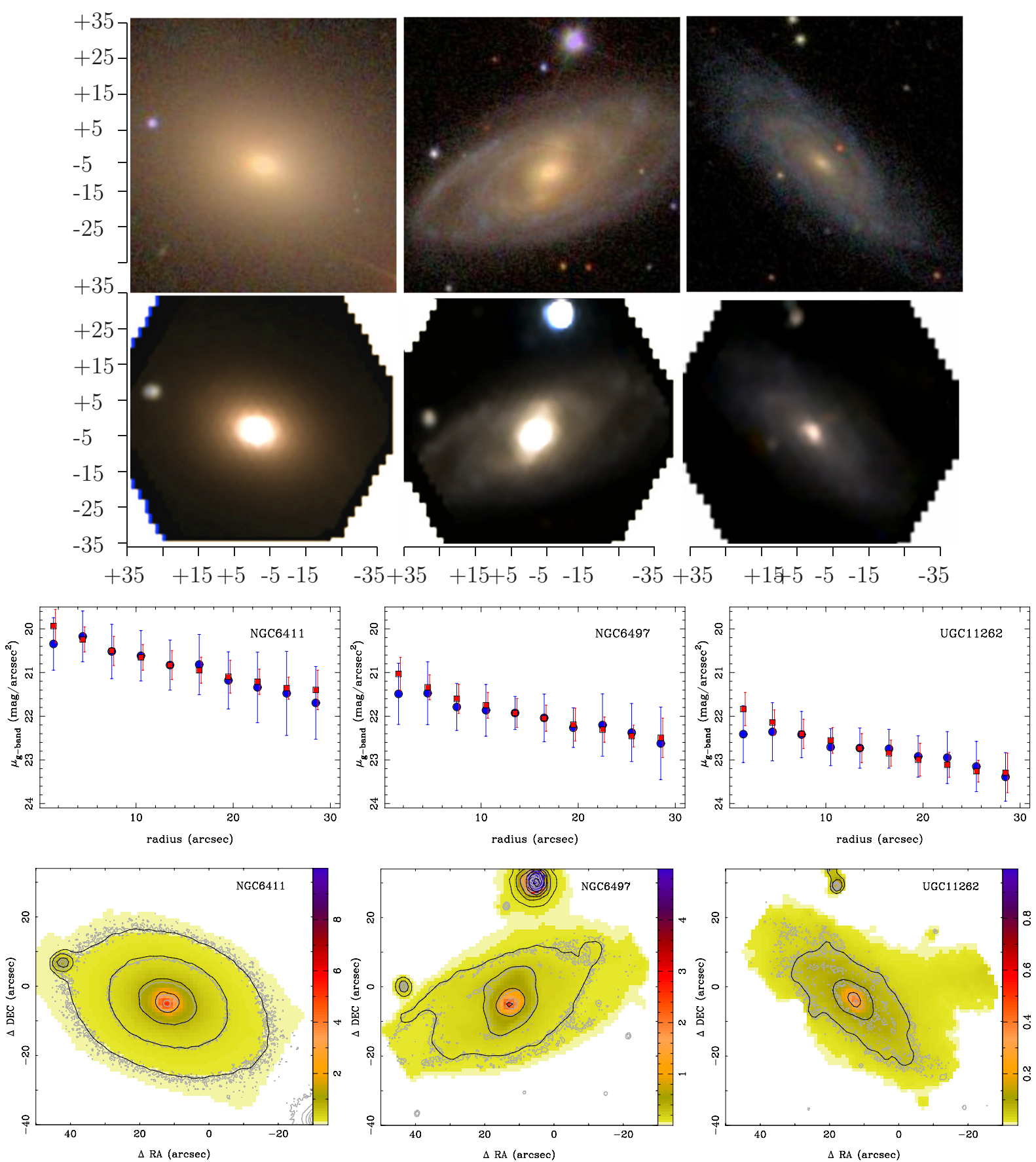

Fig. 14. Three-color images from the SDSS imaging survey (first panel), vs. those obtained from the V500 CALIFA data (second panel), for the same objects shown in Fig. 7: NGC 6411 (left), NGC 6497 (middle) and UGC 11262 (right). For the CALIFA data, $u, g$ and $r$-band images were synthesized from the corresponding datacubes. The third panel shows a comparison between the azimuthal averaged surface brightness profiles at the $g$-band, derived from synthesized images created from the V500 datacubes (blue circles) and the SDSS ones (red squares). The bottom panel shows the synthesized $g$-band image, color-scaled, together with two logarithm-scaled counter plots: one corresponding to the SDSS $g$-band image, in grey color, and the other corresponding to the synthesized image, in black.

the complete sample and spectroscopic information for a considerable subsample of the objects $(\sim 2 / 3)$. A simple quality test of the obtained data is thus to compare them with the SDSS archive data. A collection of archive auxiliary data and an analysis of the properties of the mother sample will be presented in a future paper.

A first sanity check that we perform on all reduced data is to visually inspect how they look compared to the SDSS images. For each datacube we synthesize the corresponding $u$ (for both the V500 and V1200 dataset), $g$, and $r$-band (for the V500 data) images, by convolving the cubes with the corresponding transmission curve, following the same procedure described previously for the photometric recalibration. These images are compared visually with the corresponding images in the SDSS imaging survey. Some simple problems with the data acquisition (e.g., off-centering, error in the scaling between pointings, error in the dithering pattern...), show up clearly in this visual inspection. Figure 14 illustrates the procedure, showing, for the same three objects shown in Fig. 7, a comparison between the threecolor images derived using both the SDSS and the CALIFA data. 

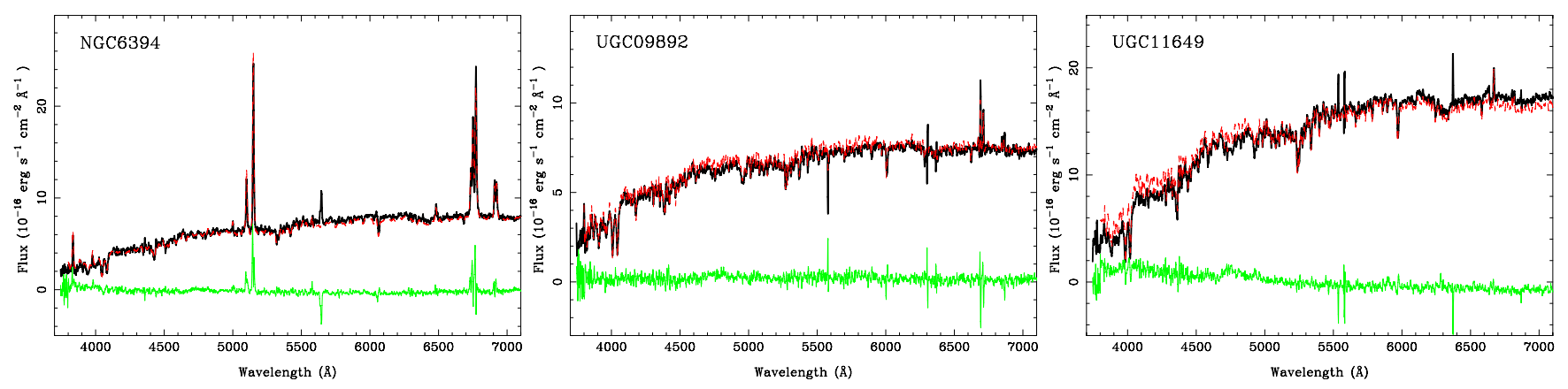

Fig. 15. Comparison of the central 5" spectrum extracted from the V500 (thick back solid-line) grating datacubes with the corresponding spectrum obtained by the SDSS (thick red dashed-line), and the difference between both spectra (thin green solid-line). There are the only three galaxies in the presented sample with SDSS spectra: NGC 6394, UGC 09892 and UGC 11649 (from left to right).

There is a clear morphological agreement between both image sets. Most of the structures seen in the SDSS images are clearly identified in the CALIFA data, in particular for the two brighter objects.

Despite this qualitative agreement, there are more quantitative comparisons to illustrate the agreement with previously published broad-band images across the FoV (e.g., Sánchez et al. 2011). A simple one is to compare the surface brightness profiles. We extracted the surface brightness profiles for the considered $g$-band images, both synthesized from the V500 datacubes and obtained from the SDSS survey. The profiles were obtained by averaging the intensity within consecutive elliptical rings of $3^{\prime \prime}$ width, using the ellipticity and position angle listed in the SDSS-NYU catalogue for each object, and centred in the peak intensity of each image. The derived profiles are plotted in Fig. 14, in the third panel. For each object the surface brightness profile derived from the $g$-band images derived from the datacubes is shown (blue solid circles), together with the same profile derived from the SDSS data (red solid squares). The error bars indicate the standard deviation around the average intensity. There is good agreement between both profiles at all the radii. In addition to the surface brightness profiles, we include in the figure a comparison between the logarithm-scaled counter-plots derived from the SDSS $g$-band images and the corresponding ones synthetized from the CALIFA datacubes. The largest differences are seen in the inner $\sim 3^{\prime \prime}$ radius, where the datacubes have a flatter distribution than found using higher resolution images. This is expected since in some cases the datacubes have an incomplete coverage of the galaxy, due to the hexagonal pattern of the IFU. Within these two radii, the standard deviation of the differences between the surface brightness is $\sim 0.13$ mag. This comparison illustrates the accuracy of the flux-calibration across the FoV.

We also perform a cross-check using the spectra. For each object with an SDSS spectrum, a combined spectrum within a $5^{\prime \prime}$ diameter aperture is extracted from the V500 grating datacubes, centered on the intensity peak of the galaxy in the $V$-band. This aperture-extracted spectrum is selected to match the aperture of the SDSS fibers $\left(\sim 3^{\prime \prime}\right)$, taking into account possible errors in the centroiding accuracy of these fibers and seeing /resolution effects. Slight differences due to seeing and resolution are expected, as illustrated in Fig. 14, bottom panel. In any case, no significant differences are found when slightly different apertures (3-6") are selected, apart from the natural scaling between the spectra. Although the fraction of objects within the CALIFA mother sample with SDSS spectra is $\sim 60 \%$, for the particular dataset currently observed there were only 3 objects out of 21 with published SDSS spectra. The resolution of the SDSS spectra was degraded to match that of the V500 CALIFA data. Finally, a constant scaling factor was applied to the SDSS spectra to compensate for the differences in apertures.

Figure 15 shows the comparison between the apertureintegrated spectra and the corresponding SDSS spectra for these three objects, including the residual after the subtraction of one from the other. Both spectra agree to within $\sim 16 \%$ (rms of the residual) over the full wavelength range. Although the comparison sample is too small to derive statistical results, it is clear that the stronger differences are found at the wavelengths of the emission lines (e.g., NGC 6394). These differences are mostly due to a non-perfect matching of the spectral resolutions (which change along the wavelength in both cases), rather than to a real difference in the intensity of each emission line (as we will show later). A slight difference is also found in the blue-to-red spectral shape of UGC11649, which is clearly seen in the distribution of the residual spectra, although both spectra match within the expected errors (taking into account our blue-to-red spectrophotometric accuracy).

In summary, these preliminary quality checks show that:

- The accuracy of the wavelength calibration corresponds to $\sim 8 \mathrm{~km} \mathrm{~s}^{-1}$ for the V500 ( 3 $\mathrm{km} \mathrm{s}^{-1}$ for the V1200), along the covered wavelength range.

- The final spectral resolution in FWHM is $\sim 6.5 \AA$, i.e., $\sigma \sim$ $150 \mathrm{~km} \mathrm{~s}^{-1}$ for the $\mathrm{V} 500\left(\sim 2.7 \AA\right.$ i.e., $\sigma \sim 85 \mathrm{~km} \mathrm{~s}^{-1}$ for the V1200). It has been homogenized across the FoV and along the wavelength range, taking into account the spectral and spatial variations.

- The sky subtraction has a typical residual of $\sim 2 \%$ of the original flux of the night-sky spectrum.

- The flux calibration is anchored to the SDSS photometry. The final photometry is accurate to $\sim 8 \%$ with respect to SDSS, with variation across the FoV of $\sim 0.13 \mathrm{mag} / \mathrm{arcsec}^{2}$.

- The datacubes reach a $3 \sigma S / N$ depth at an average surface brightness of $\sim 23.0 \mathrm{mag} / \operatorname{arcsec}^{2}$ for the V500 data ( $22.7 \mathrm{mag} / \operatorname{arcsec}^{2}$ for the V1200 data), with $\sim 50-90 \%$ of the FoV above this $S / N$ limit (with an average of $\sim 70 \%$ of the FoV covered). It is estimated that $\sim 90 \%$ of the survey datacubes will reach this depth.

In summary, the data have the quality foreseen in the proposal ${ }^{7}$, which will allow us to study the spatial distribution of different spectroscopic properties, including: (1) the ionized gas emission

${ }^{7}$ http://www.caha.es/CALIFA/Accepted_Proposal.pdf 
lines from [OII] $\lambda 3727$ to $[\mathrm{SII}] \lambda 6731$, (2) the stellar population features from D4000 to Fe5335 and (3) the gas and stellar kinematics, with a resolution of $\sim 85 \mathrm{~km} \mathrm{~s}^{-1}$. We conclude that we are on track for providing high-quality, well-calibrated and wellcharacterized reduced data to the users of the CALIFA survey.

\section{Verification of scientific usability}

In this section we describe a showcase analysis performed on the CALIFA data available to date, with the double purpose of verifying that we will be able to reach our science goals and of illustrating the information contained in the data. It is beyond the scope of this article to perform a detailed analysis of these data to achieve any of the goals of the survey, which in any case require a statistical sample of objects to be observed.

\subsection{Continuum modeling and gas decoupling}

To extract the information contained in the datacubes, the absorption spectra must be separated from the emission lines for each of the analyzed spectra (e.g., Sánchez et al. 2011). Several different tools have been developed to model the underlying stellar population, effectively decoupling it from the emission lines (e.g., Cappellari \& Emsellem 2004; Cid Fernandes et al. 2005; Ocvirk et al. 2006; Sarzi et al. 2006; Walcher et al. 2006; Sánchez et al. 2007a; Koleva et al. 2009; MacArthur et al. 2009; Sánchez et al. 2011). Most of these tools are based on the same principles. It is assumed that the stellar emission is the result of the combination of different (or a single) single-stellar populations (SSP), and/or the result of a particular star-formation history. The stellar continuum is redshifted by a certain systemic velocity, broadened and smoothed due a certain velocity dispersion and attenuated due to a certain dust content. Once the stellar continuum is determined, it is subtracted from each spectrum. This provides a pure emission line spectrum, including the information of the ionized gas. The intensity, systemic velocity and velocity dispersion of each emission line can be derived by, for example, fitting with a set of Gaussian functions. In general it is known that the information derived from emission lines is much more accurate and stable than that derived for stellar populations (see Walcher et al. 2011, for a review of the state of the art). However, an accurate decontamination of the stellar continuum is required to measure emission line ratios, such as the $[\mathrm{OIII}] / \mathrm{H} \beta$ ratio, which allow us to interpret the data in terms of physical processes e.g. ionization source.

It is beyond the scope of this article to analyze in detail the nature of stellar populations in the considered objects, since this will be one of the major goals of the full CALIFA project. Therefore, for the current demonstration we perform a simple modelling of the continuum emission. We use the routines described in Sánchez et al. (2011) and Rosales-Ortega et al. (2010). These routines fit the underlying stellar population combining linearly a set of stellar templates within a multi-SSP model. They provide us with a number of parameters describing the physical components of the stellar populations (e.g., luminosity-weighted ages, metallicities and stellar dust attenuation, together with the systemic velocity and velocity dispersion), and the properties of the analyzed emission lines (intensity, velocity and velocity dispersion). No detailed comparison between the different available tools or a detailed error analysis is considered here. A simple SSP template grid was adopted, consisting of three ages $(0.09$, 1.00 and $17.78 \mathrm{Gyr})$ and two metallicities $(Z \sim 0.0004$ and 0.03$)$. The models were extracted from the SSP template library provided by the MILES project (Vazdekis et al. 2010). This library is most probably too simple to describe in detail the nature of all the stellar populations included in the current dataset. However, it covers the space of possible stellar populations, and it allows us to obtain reliable information on the ionized gas. This fitting procedure has been adopted to analyze different apertureextracted and single spectra within the datacubes, as we will describe in the following sections.

Figure 16 illustrates the results of the fitting procedure for the six galaxies also shown in Figs. 7 and 15. In each panel the solid black line represents the integrated spectrum of the corresponding galaxy (i.e., 30" diameter aperture spectra), together with the best multi-SSP model for the stellar population (green line), and the gas emission recovered (red line).

\subsection{Comparing the results derived by CALIFA and SDSS}

The SDSS has become a well-appreciated standard for spectroscopic data and also has been used to select the mother sample of the CALIFA survey. It is therefore of interest to make a quantitative comparison of the information provided by both datasets. To this end we analyze both spectra (see Sect. 6.6) using the fitting algorithm described in Sect. 7.1. Table 2 summarizes the results of this analysis. For each galaxy and each spectral dataset it lists the luminosity-weighted age, metallicity, dust attenuation and velocity dispersion, derived from the analysis of the stellar population. It also lists diagnostic emission line ratios, such as $\mathrm{H} \alpha / \mathrm{H} \beta,[\mathrm{OIII}] / \mathrm{H} \beta,[\mathrm{NII}] / \mathrm{H} \alpha$ and $[\mathrm{SII}] 6717 / 6731$ line ratios. The parameters describing the stellar populations match reasonably well, considering that a typical error of $\sim 20-30 \%$ is expected in all of them (Sánchez et al. 2011). The properties of the ionized gas match each other within the reported errors.

Despite the small number of compared spectra, the good agreement in all derived spectroscopic parameters indicates that the current data provided by CALIFA have, on average and per fiber, a similar information content to that provided by the SDSS. The major advantage of CALIFA is the spatial coverage of the spectral information.

\subsection{Central vs. total spectra}

One of the science goals of CALIFA is to allow the extraction of observed spectra of particularly interesting regions or the full galaxy to produce integrated spectra using the IFU as an adaptive aperture spectrograph. This technique has been used by Rosales-Ortega et al. (2010) to produce aperture selected spectra of HII regions within the galaxies of the PINGS survey, or in Sánchez et al. (2011) to derive the integrated spectrum of the complete galaxies.

The full-aperture integrated spectra can be used to derive, for the first time, the real integrated spectroscopic properties of these galaxies, as opposed to previous studies that attempted to describe average properties by the analysis of individual spectra taken in different regions. The most similar approach would be the drift-scanning technique (e.g. Moustakas \& Kennicutt 2006, and part of the ancillary data of the SINGS survey), although in those studies the fraction of the galaxy covered by the spectra was smaller than that of CALIFA. Another advantage of an IFU with respect to the drift-scan technique is that the former allows a comparison between the integrated and the spatially resolved properties of the galaxy, or the integrated spectra of particular regions.

In this section we analyze two spectra derived for each galaxy for different apertures, using the V500-grating dataset. 

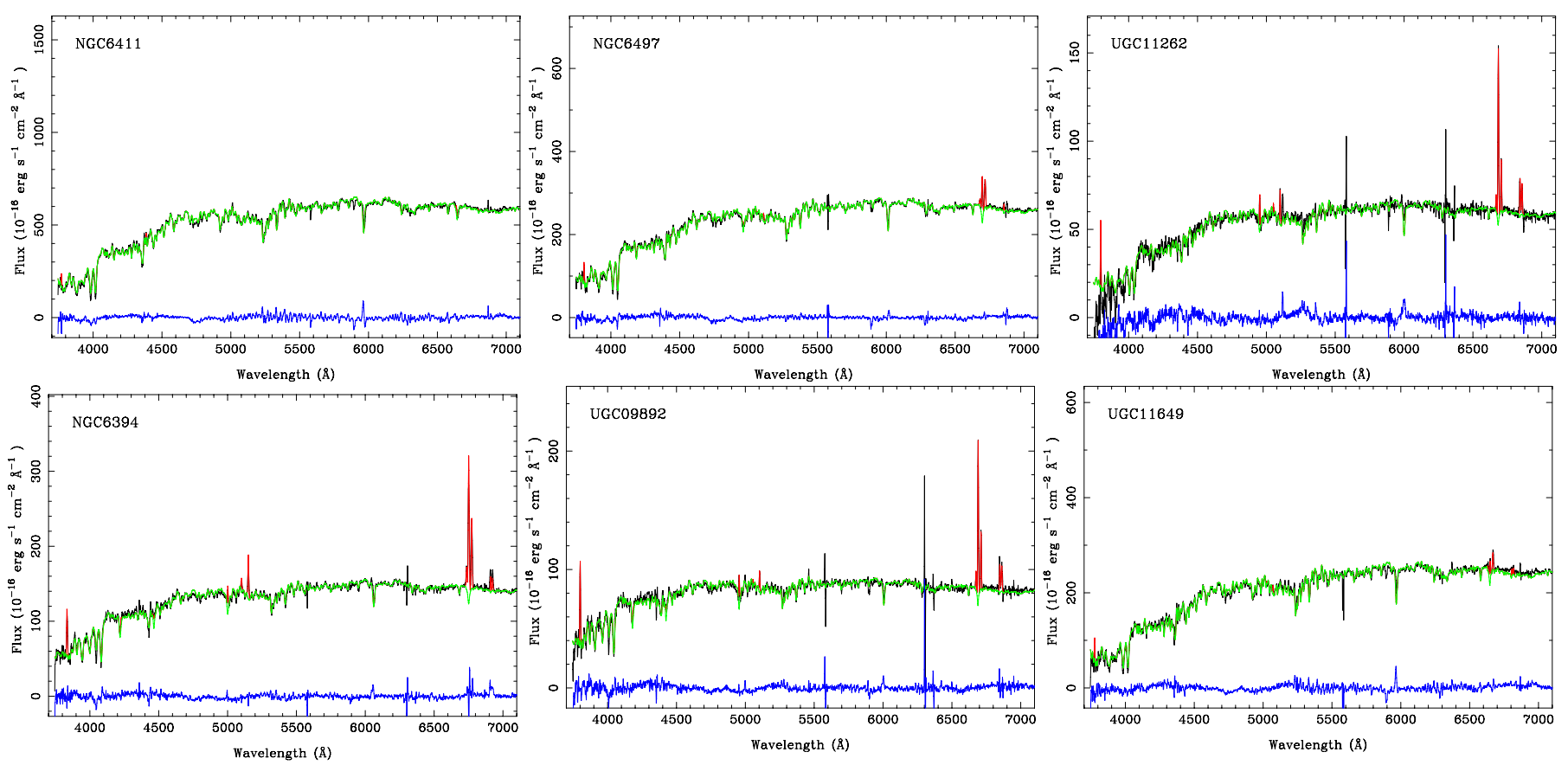

Fig. 16. Results of the fitting procedure applied to each single spectrum contained in the dataset, described in the text, for the six galaxies shown in Figs. 7 and 15. Each panel shows the integrated spectrum of the corresponding galaxy (solid-black line) derived by coadding the spectra within a 30" diameter aperture centered on the peak emission in the $V$-band. The green line shows the best-fit multi-SSP stellar population, while the red line shows the best model derived for the emission of ionized gas. The blue line is the residual from the fit.

Table 2. Comparison of the PPAK central spectrum with the SDSS single-fiber spectrum.

\begin{tabular}{lcccrcrrr}
\hline \hline NAME & \multicolumn{3}{c}{ PPAK central spectrum } & \multicolumn{5}{c}{ SDSS spectrum } \\
& $\log _{10}$ (Age) Gyr & $Z$ & $A_{\mathrm{V}}$ & $\sigma_{\mathrm{v}} \mathrm{km} \mathrm{s}^{-1}$ & $\log _{10}($ Age $) \mathrm{Gyr}$ & $Z$ & $A_{\mathrm{V}}$ & $\sigma_{\mathrm{v}} \mathrm{km} \mathrm{s}^{-1}$ \\
\hline NGC 6394 & 1.1 & 0.016 & 0.7 & 139 & 1.3 & 0.016 & 0.5 & 141 \\
UGC 09892 & 0.7 & 0.019 & 0.4 & 116 & 0.8 & 0.021 & 0.3 & 144 \\
UGC 11649 & 1.3 & 0.024 & 0.2 & 228 & 1.0 & 0.024 & 0.2 & 215 \\
\hline & $\mathrm{H} \alpha / \mathrm{H} \beta$ & {$[\mathrm{OIII}] / \mathrm{H} \beta$} & {$[\mathrm{NII}] / \mathrm{H} \alpha$} & {$[\mathrm{SII}] \lambda \frac{6717}{673 !}$} & $\mathrm{H} \alpha / \mathrm{H} \beta$ & {$[\mathrm{OIII}] / \mathrm{H} \beta$} & {$[\mathrm{NII}] / \mathrm{H} \alpha$} & {$[\mathrm{SII}] \lambda \frac{6717}{6731}$} \\
\hline NGC 6394 & $6.9 \pm 2.0$ & $8.2 \pm 2.2$ & $1.1 \pm 0.1$ & $1.2 \pm 0.8$ & $6.6 \pm 1.7$ & $10.3 \pm 2.4$ & $1.3 \pm 0.1$ & $1.0 \pm 0.1$ \\
UGC 09892 & $8.0 \pm 3.5$ & $0.7 \pm 0.6$ & $0.5 \pm 0.1$ & $0.7 \pm 0.1$ & - & - & $0.5 \pm 0.1$ & $1.5 \pm 0.7$ \\
UGC 11649 & - & - & $1.1 \pm 1.1$ & $1.3 \pm 0.2$ & $3.8 \pm 4.1$ & $2.2 \pm 2.8$ & $1.1 \pm 0.5$ & $1.5 \pm 0.6$ \\
\hline
\end{tabular}

One is a $5^{\prime \prime}$ diameter aperture spectrum, centered on the peak intensity in the $V$-band. This spectrum is representative of the central region of each galaxy, and is similar to the one obtained by the SDSS survey (see Sect. 6.6). The other is a 30" diameter aperture spectrum, an aperture that contains most fibers above the $5 \sigma$ detection limit for each galaxy. The latter spectrum is representative of the integrated properties of each galaxy. Both spectra have similar $S / N(\sim 40$, at $\sim 5000 \AA)$. This is due to the fact that the central spectrum samples areas of much higher $S / N$, despite its smaller aperture, while the total spectrum samples areas of lower $S / N$, but with a larger aperture. On average, the $5^{\prime \prime}$ aperture contains $10 \%$ of the flux encircled by the $30^{\prime \prime}$ aperture. We applied the above fitting technique described in Sect. 7.1 to both spectra, deriving the main properties of both the stellar population and the ionized gas. An example of typical integrated spectra, and their modelling, was already shown in Fig. 16.

\subsubsection{Stellar populations}

Gradients and, more generally, spatial variations of stellar population properties have been observed in galaxies, both early types (e.g. Kuntschner et al. 2006) and late types (e.g. MacArthur et al. 2004). CALIFA will allow the mapping of these variations for the first time over a statistically representative sample of galaxies, unbiased in terms of morphology and mass/luminosity. The detailed modeling required to this goal is beyond the scope of this paper and will be presented in forthcoming dedicated works. Here we just illustrate the potential of the dataset using either model-independent measurements, such as the spectral indices, or the output of the simple fitting technique described in Sect. 7.1.

It is known (e.g. Trager et al. 2000; Gallazzi et al. 2005) that spectral indices can be used to infer stellar population parameters such as age, metallicity and $\alpha$ enhancement. They provide robust, model-independent, information, complementary to that provided by fitting the full spectrum with SSP templates as described in Sect. 7.1. For the current analysis, we have explored the D4000 index (i.e. the $4000 \AA$ break) and the $\mathrm{H} \delta$ index (which represents the equivalent width of the $\mathrm{H} \delta$ line), as tracers of stellar age, and $[\mathrm{MgFe}]$ as a tracer of stellar metallicity. For this later index, we adopted the formula:

$$
[\mathrm{MgFe}]=\sqrt{\mathrm{Mg} b\left(0.72 \mathrm{Fe}_{5270}+0.28 \mathrm{Fe}_{5335}\right)} .
$$

The indices were measured for both the central and integrated spectra, once decontaminated by the ionized gas emission and normalized to the standard Lick/IDS resolution, using the scripts included in FIT3D, described in Sánchez et al. (2007b). 
S.F. Sánchez et al.: CALIFA, the Calar Alto Legacy Integral Field Area survey. I.

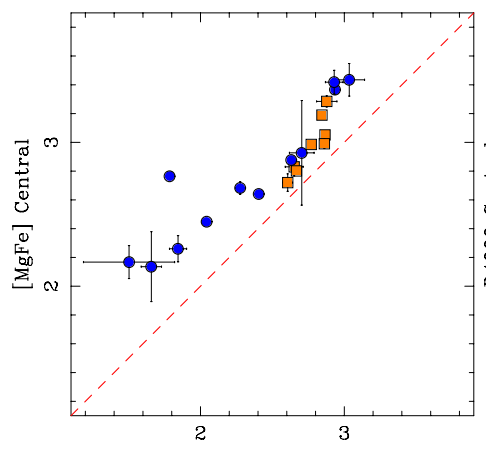

$[\mathrm{MgFe}]$ Integrated

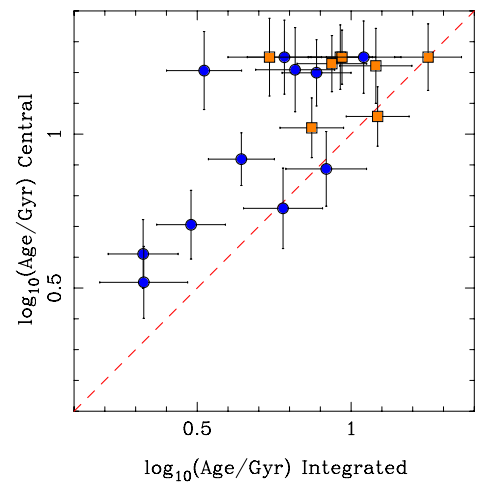

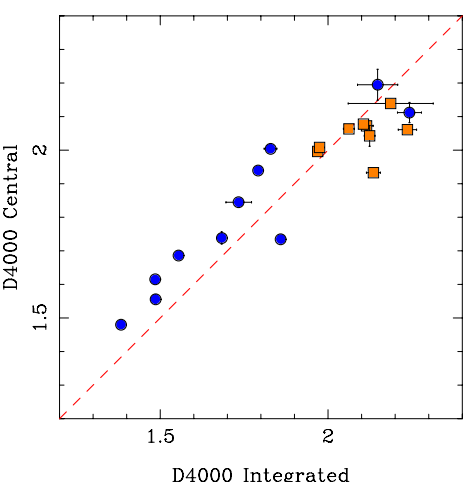

D4000 Integrated

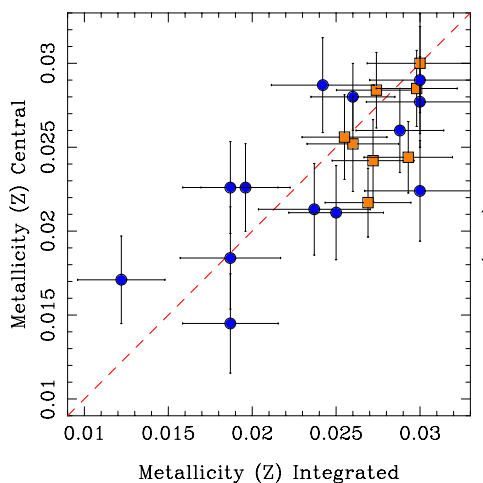

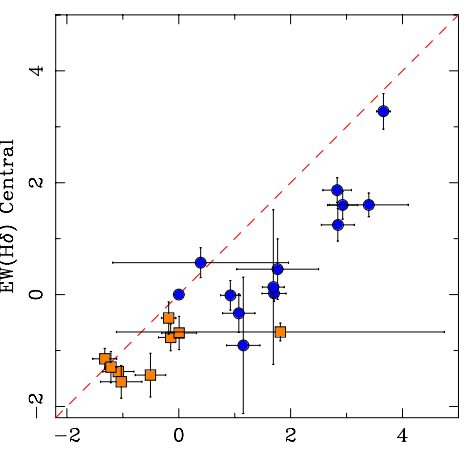

$\mathrm{EW}(\mathrm{H} \delta)$ Integrated

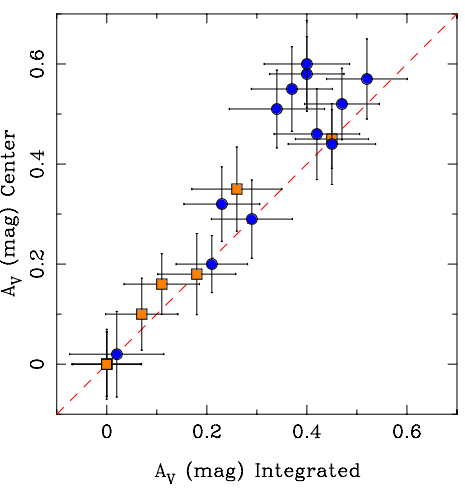

Fig. 17. Comparison between the properties derived for the stellar populations on the basis of the analysis of the stellar indices and the results of the multi-SSP fitting procedure, for the central and integrated spectra. From from left to right and top to bottom, each panel shows the comparison of the $[\mathrm{MgFe}]$ index, the $\mathrm{D} 4000$ parameter, the equivalent width of $\mathrm{H} \delta$, the luminosity-weighted age, the luminosity-weighted metallicities, and the dust attenuation of the stellar component. The orange squares indicate the parameters for the early-type galaxies ( $E$ or $S 0$ ones), while the blue circles indicate the parameters for the remaining galaxies.

Figure 17 shows a comparison of the properties derived for the underlying stellar populations for both spectral apertures. It includes the results from the analysis of the indices, i.e., the agesensitive D4000 and $\mathrm{H} \delta$ indices and the metal-sensitive [ $\mathrm{MgFe}]$ index, and the physical parameters luminosity-weighted age, metallicity and dust attenuation, estimated by fitting SSP templates to the full spectrum. The galaxy UGC 11262 has been excluded due to its lower $S / N$ level in the continuum due to technical problems during the observations. These problems affect the shape of the continuum and therefore the parameters derived by the fitting procedure, although they affect neither the stellar indices nor the parameters of the emission lines.

Figure 17 shows the correlations between central (within 5 arcsec) and total integrated indices. Red dashed lines are used to display the one-to-one correlation and guide the eye. For all indices we find tight correlations between central and total values. However, we find systematic offsets with respect to the oneto-one line, which indicate gradients: in particular, for the stellar population indices, $i$ ) $[\mathrm{MgFe}]$ central is always significantly higher than total, thus indicating negative metallicity gradients in all galaxies; $i i) \mathrm{H} \delta$ is typically stronger in the integrated spectra than in the central ones, thus indicating that young stellar populations are mainly found in the outskirts of galaxies; iii) D4000 has typically flatter radial distribution, with some indication for positive (age) gradients for the oldest galaxies (those with the strongest D4000), and vice versa for the younger ones.

Similar trends are appreciated in the parameters derived from the SSP fitting procedure. On average the central stellar populations are slightly older than the integrated ones, and they suffer a stronger or equal dust attenuation. The metallicity shows no clear trend, with values randomly distributed around the oneto-one relation. Maybe the selected library template, with only two extreme metallicities in the grid, is not good enough to sample this parameter properly, which is better represented by the $[\mathrm{MgFe}]$ index. Differences are also appreciated among the different galaxy types. The stellar component of the early-type galaxies $(E$ or $S O)$ is, in all the cases, dominated by old ( $>7 \mathrm{Gyr}$, high $\mathrm{D} 4000$ and low $\mathrm{H} \delta$ values) and metal rich $(Z>0.025$, hight $[\mathrm{MgFe}]$ values) populations. In addition, very low dust attenuation is found in both the integrated and central spectra $\left(A_{\mathrm{v}}<0.5 \mathrm{mag}\right.$, with a mean value of $\sim 0.2 \mathrm{mag}$ ). On the other hand, the late-type galaxies show a wider variety of properties in their stellar populations.

The information provided by the indices and the SSP analysis is complementary, but leads to similar conclusions, in general. Figure 18 shows a comparison between each stellar population index and its corresponding parameter derived by the SSP analysis, for the integrated spectra (similar results are found for the central spectra). As expected there is a clear correlation between the age-sensitive indices (D4000 and $\mathrm{H} \delta$ ) and the luminosity weighted ages, on one hand, and a weaker trend between the $[\mathrm{MgFe}]$ and the luminosity weighted metallicity, on the other hand. The larger scatter in this relation is most probably due to an incorrect sampling of the metallicities in the over-simplistic stellar population library grid adopted for the current analysis.

Although a more detailed analysis is required to understand the described trends, these preliminary results seem very encouraging, illustrating the kind of studies that can be done when applying more refined analysis techniques over the full CALIFA sample. 

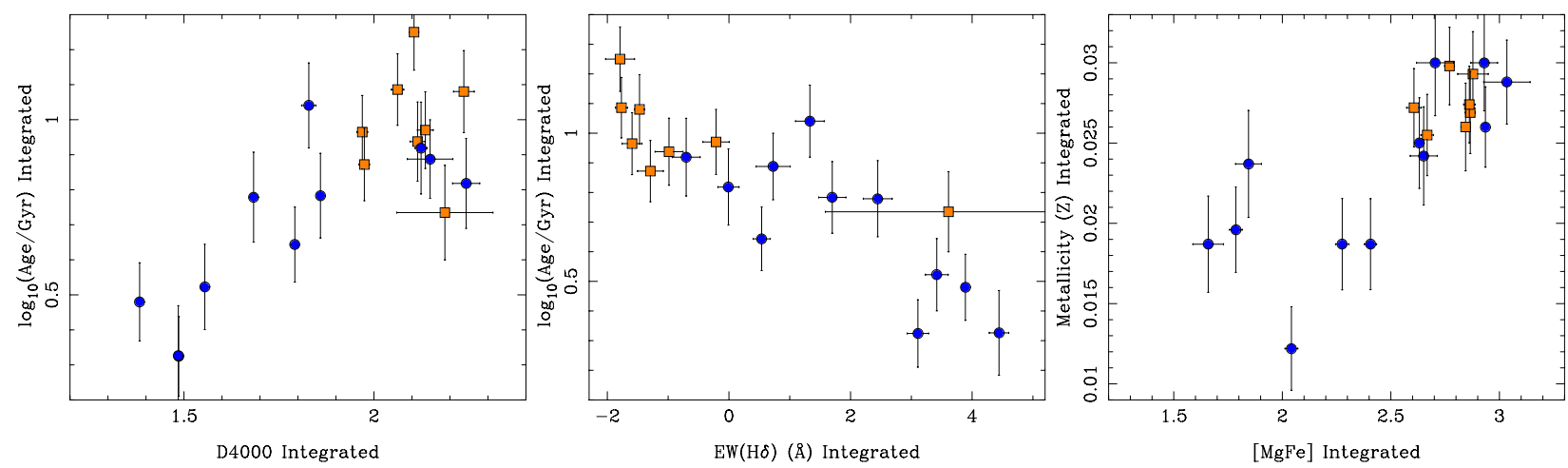

Fig. 18. Comparison between the properties derived for the stellar populations on the basis of the multi-SSP fitting procedure (Age and Metallicity) and the corresponding properties derived on the basis of the analysis of the stellar indices (D4000, $\mathrm{EW}(\mathrm{H} \delta)$ and $[\mathrm{MgFe}])$, for the integrated spectra. The symbols are defined in Fig. 17.
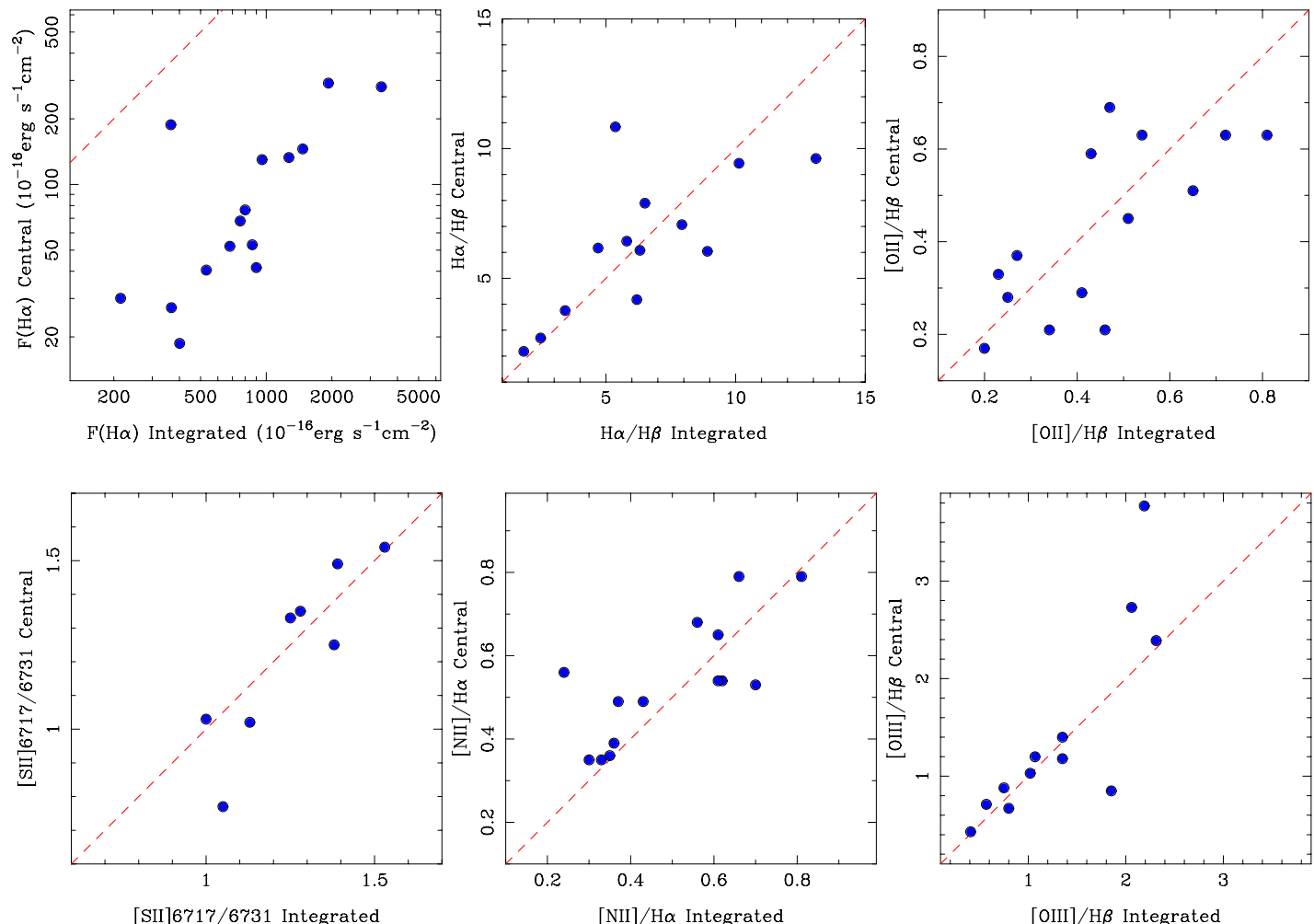

Fig. 19. Comparison between the gas parameters derived for the central and integrated spectra using the fitting procedure described in the text. From from left to right and top to bottom each panel shows the comparison between the $\mathrm{H} \alpha$ flux, and the $\mathrm{H} \alpha / \mathrm{H} \beta$, [OII] $\lambda 3727 / \mathrm{H} \beta$, [SII] $]$ 6717/[SII] $\lambda 6731$, $[\mathrm{NII}] \lambda 6584 / \mathrm{H} \alpha$ and $[\mathrm{OIII}] \lambda 5007 / \mathrm{H} \beta$ line ratios. Only values with a $S / N>3$ for both spectra have been included in each plot.

\subsubsection{Ionized gas properties}

Figure 19 shows a comparison between the emission line fluxes and ratios of the ionized gas in the integrated and central spectra, derived using the previously described fitting procedure (Sect. 7.1). The figure includes the observed flux intensity of the $\mathrm{H} \alpha$ emission line, and different line ratios between some of the strongest emission lines, including $\mathrm{H} \alpha / \mathrm{H} \beta$, [OII] $/ \mathrm{H} \alpha$, [SII]6717/6731, [NII]/H $\alpha$ and $[\mathrm{OIII}] / \mathrm{H} \beta$. None of the plotted values have been corrected for dust attenuation, and only those with a $S / N>3$ have been included in each plot. $\mathrm{H} \alpha$ gas emission is detected in the integrated spectra of 15 galaxies, and in the central spectra of 16. As expected, most of the galaxies without detected ionized gas emission (i.e. $F(\mathrm{H} \alpha)<3 \sigma$ ), either in the central or in the integrated spectra are early-type galaxies (E and $\mathrm{S} 0$ ).

The comparison illustrates the importance of mapping the full optical size of galaxies to derive the average properties of the ionized gas. For example, the $\mathrm{H} \alpha$ intensity, as an additive property, is much larger in the integrated spectra than in the central aperture. The differences in other non-additive properties (e.g., the $\mathrm{H} \alpha / \mathrm{H} \beta$ line ratio), illustrate changes in the physical conditions of the gas from the inner to the outer regions.

The plotted emission line ratios can be used to distinguish the ionizing source of the gas associated with each region. In particular, the $[\mathrm{OIII}] / \mathrm{H} \beta$ and $\mathrm{NII} / \mathrm{H} \alpha$ can be used as a diagnostic probe which is almost insensitive to the dust attenuation. 

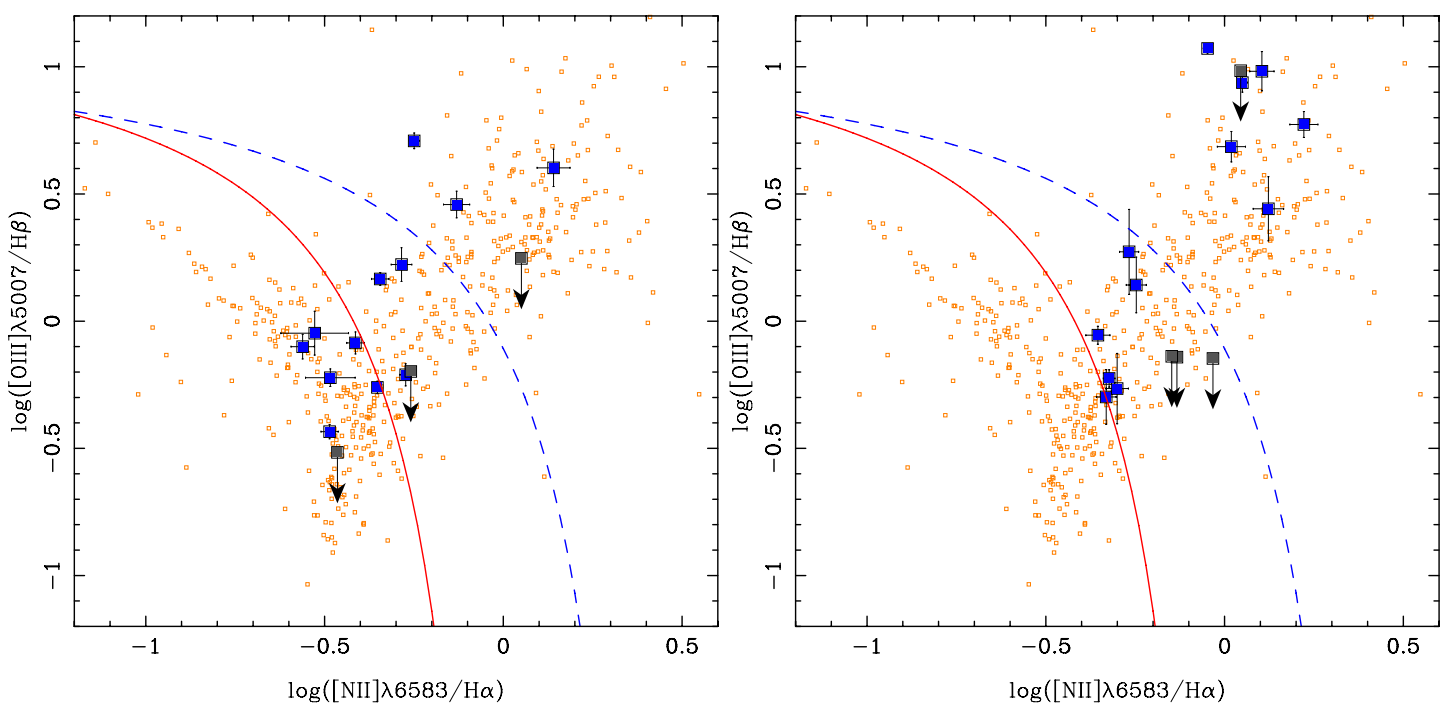

Fig. 20. [OIII] $\lambda 5007 / \mathrm{H} \beta$ vs. [NII] $\lambda 6583 / \mathrm{H} \alpha$ diagnostic diagram for integrated (left panel) and central (right panel) spectra of the currently observed CALIFA sample. Upper limits are indicated with an arrow, if only one of the two ratios could be accurately determined. We also plot data derived from the SDSS spectra of the CALIFA mother sample (orange open squares). The Kauffmann et al. (2003) (blue dashed-line) and Kewley et al. (2001) (red solid-line) demarcation curves are usually invoked to distinguish between SF galaxies and AGN with an intermediate region in between.

Figure 20 shows the classical BPT diagram (Baldwin et al. 1981; Veilleux \& Osterbrock 1987) for both the integrated (left-panel) and central (right-panel) spectra for the galaxies with detected ionized gas. We included in the figure the corresponding line ratios estimated from the 496 SDSS spectra of the CALIFA mother sample with clearly detected ionized gas. The diagram shows the typical butterfly structure, with a left branch corresponding to HII regions powered by the ionizing radiation of young OB stars (i.e., star forming regions), while the branch on the right side is usually attributed to ionization by an AGN. We include the theoretical boundary between ionization from stars (SF) and from an AGN from Kewley et al. (2001) as well as the one empirically derived by Kauffmann et al. (2003). Objects are thus classified as star forming (SF), AGN-ionized (AGN), or intermediate $(\mathrm{SF} / \mathrm{AGN})$ if they lie between the empirical boundary and the theoretical one. Table 3 includes the result of this classification. Note that the current classification scheme has not considered the possibility of ionization due to post-AGB stars as suggested by several authors (e.g., Trinchieri \& di Serego Alighieri 1991; Binette et al. 1994; Stasińska et al. 2008; Cid Fernandes et al. 2010; Sarzi et al. 2010). It is beyond the scope of the current study to analyze that possibility in detail, although it may lead to the reclassification of the ionized sources presented here.

For the integrated spectra with both line ratios detected (+upper limits) we find that $6(+1)$ galaxies are classified as SF, 3(+1) as AGN and 3(+1) as intermediate. On the other hand, based on the properties of the central spectra, $6(+1)$ galaxies are classified as pure AGN and $6(+3)$ as intermediate. The fraction of AGN detected in this first reduced sub-sample observed so far by CALIFA is much larger than the one expected on the basis of the analysis of the SDSS spectra (i.e. $\sim 1 / 3$ of the objects), as is clearly seen in the figure. Targets were selected from the mother sample purely based on observability. Furthermore, we have so far not found significant differences between the properties of the SDSS and CALIFA central spectra. Therefore, most probably this effect is a result of small number statistics.

That the fraction of galaxies classified as SF is larger for integrated spectra than for central spectra is of course expected as an aperture effect. It is one of the design goals of CALIFA to quantify this effect.
In addition to the determination of the main ionization source, the emission line spectrum can be used to derive properties of the ionized gas. The dust attenuation $\left(A_{\mathrm{V}}\right)$ of emission lines along the line of sight through a galaxy can be derived from the Balmer decrement, assuming an extinction law (e.g. Cardelli et al. 1989) and comparing the theoretical vs. observed $I(\mathrm{H} \alpha) / I(\mathrm{H} \beta)$ ratio. Table 3 lists the derived dust attenuation for each object, for both the integrated and central spectra ${ }^{8}$.

Extinction-corrected line fluxes can be used to derive the star formation rate $(S F R)$, based either on the $\mathrm{H} \alpha$ or [OII] 3727 line intensities. The $S F R$ s can be derived adopting the classical relation between this parameter and the luminosity of both emission lines (Kennicutt 1998). Note that the absolute luminosities at the distances involved in CALIFA are insensitive to the assumed cosmology 9 .

Table 3 includes the results of this calculation for those galaxies in which the ionization is not dominated by an AGN. In most of the cases the SFRs derived using both lines are consistent within the errors. In a few cases $(\sim 10 \%)$, a much higher $S F R$ is found from [OII] than using $\mathrm{H} \alpha$ (e.g. UGC 12185 for the integrated spectrum, and UGC10710 for the central one). In these cases the observed [OII]/H $\alpha$ ratio does not seem to be abnormally high. An inspection of these spectra shows that for such cases the correction of $\mathrm{H} \beta$ by the underlying stellar absorption is particularly high, which may affect the derivation of the dust attenuation and most probably produces an over-correction of the [OII] flux. Galaxies for which the $[\mathrm{OII}] / \mathrm{H} \alpha$ ratio is higher than the average are located in the intermediate region of the BTP diagram, between the pure SF and pure AGN ionization regimes.

The emission lines present within the spectral range of CALIFA allow us to examine other properties of the ionized gas, such as the hardness of the ionization, typically characterized by the ionization parameter $U$, defined as the ratio of the density of ionizing photons to the particle density. This parameter is best determined using the ratios of emission lines of the same element originating from different ionization stages. The

\footnotetext{
${ }^{8}$ Assuming $R_{\mathrm{V}}=3.1$ and case $\mathrm{B}$ recombination: temperature $10^{4} \mathrm{~K}$, density $10^{2} \mathrm{~cm}^{-3}$; (Osterbrock 1989).

9 A $H_{0}=70 \mathrm{~km} \mathrm{~s}^{-1} \mathrm{Mpc}^{-1}$ and $q_{0}=0.5$ was adopted.
} 
Table 3. Summary of the physical properties derived from the analysis of the ionized gas for the integrated and central spectrum of each galaxy.

\begin{tabular}{|c|c|c|c|c|c|c|c|}
\hline \multicolumn{8}{|c|}{ Integrated spectrum } \\
\hline NAME & Spec. Type & $\begin{array}{c}A_{\mathrm{V}} \\
(\mathrm{mag})\end{array}$ & $\begin{array}{l}S F R_{\mathrm{H} \alpha} \\
M_{\odot} \mathrm{yr}^{-1}\end{array}$ & $\begin{array}{l}S F R_{[\mathrm{OII}]} \\
M_{\odot} \mathrm{yr}^{-1}\end{array}$ & $\log (U)$ & $12+\log (\mathrm{O} / \mathrm{H})$ & $\begin{array}{l}\text { Electron density } \\
\mathrm{cm}^{-3}\end{array}$ \\
\hline NGC 5947 & $\overline{S F}$ & $1.2 \pm 0.1$ & $3.8 \pm 0.1$ & $3.8 \pm 0.8$ & $-3.5 \pm 0.2$ & $8.7 \pm 0.1$ & - \\
\hline UGC 09892 & $\mathrm{SF}$ & $1.3 \pm 0.2$ & $2.3 \pm 0.1$ & $3.9 \pm 0.8$ & $-3.7 \pm 0.4$ & $8.7 \pm 0.3$ & - \\
\hline NGC 6394 & SF/AGN & $2.1 \pm 0.2$ & $12.1 \pm 0.1$ & $14.1 \pm 2.8$ & $-3.2 \pm 0.3$ & $8.6 \pm 0.1$ & $214 \pm 64$ \\
\hline NGC 6497 & $\mathrm{SF} / \mathrm{AGN}$ & $1.5 \pm 0.2$ & $3.7 \pm 0.1$ & $3.4 \pm 0.7$ & $-3.5 \pm 0.4$ & $8.7 \pm 0.1$ & - \\
\hline UGC 11262 & SF & $1.5 \pm 0.2$ & $1.8 \pm 0.1$ & $1.4 \pm 0.3$ & $-3.3 \pm 0.7$ & $8.6 \pm 0.2$ & $145 \pm 43$ \\
\hline NGC 6762 & AGN & $1.5 \pm 0.2$ & - & - & - & - & - \\
\hline UGC 11680 & AGN & $3.3 \pm 0.2$ & - & - & $-3.0 \pm 0.5$ & - & - \\
\hline UGC 11694 & SF & $0.0 \pm 0.5$ & $0.3 \pm 0.1$ & $0.2 \pm 0.1$ & $-2.9 \pm 0.7$ & $8.6 \pm 0.4$ & - \\
\hline UGC 11717 & AGN & $3.3 \pm 0.4$ & - & - & $-3.6 \pm 0.2$ & - & $4571 \pm 1371$ \\
\hline UGC 11740 & SF & $1.8 \pm 0.2$ & $3.5 \pm 0.1$ & $5.1 \pm 1.0$ & $-3.5 \pm 0.5$ & $8.6 \pm 0.2$ & $525 \pm 157$ \\
\hline UGC 12185 & $\mathrm{SF} / \mathrm{AGN}$ & $2.8 \pm 0.4$ & $6.2 \pm 0.1$ & $22.1 \pm 15.0$ & $-3.6 \pm 0.6$ & $8.6 \pm 0.2$ & - \\
\hline NGC 7549 & SF & $2.4 \pm 0.1$ & $10.8 \pm 0.1$ & $10.3 \pm 0.1$ & $-3.7 \pm 0.2$ & $8.7 \pm 0.1$ & - \\
\hline \multicolumn{8}{|c|}{ Central spectrum } \\
\hline NAME & Spec. Type & $\begin{array}{c}A_{\mathrm{V}} \\
(\mathrm{mag})\end{array}$ & $\begin{array}{l}S F R_{\mathrm{H} \alpha} \\
M_{\odot} \mathrm{yr}^{-1}\end{array}$ & $\begin{array}{l}S F R_{[\mathrm{OII}]} \\
M_{\odot} \mathrm{yr}^{-1}\end{array}$ & $\log (U)$ & $12+\log (\mathrm{O} / \mathrm{H})$ & $\begin{array}{l}\text { Electron density } \\
\mathrm{cm}^{-3}\end{array}$ \\
\hline NGC 5947 & SF/AGN & $1.5 \pm 0.1$ & $0.4 \pm 0.2$ & $0.2 \pm 0.1$ & $-3.2 \pm 0.3$ & $8.7 \pm 0.1$ & - \\
\hline UGC 09892 & SF/AGN & $2.6 \pm 0.3$ & $0.2 \pm 0.2$ & $0.4 \pm 0.2$ & $-3.8 \pm 0.6$ & $8.7 \pm 0.3$ & $2512 \pm 754$ \\
\hline UGC 10710 & SF/AGN & $4.5 \pm 0.9$ & $3.9 \pm 0.1$ & $23.2 \pm 13.9$ & $-3.8 \pm 0.6$ & $8.6 \pm 0.5$ & - \\
\hline NGC 6394 & AGN & $2.9 \pm 0.3$ & - & - & $-2.8 \pm 0.1$ & - & $309 \pm 93$ \\
\hline NGC 6497 & AGN & $1.8 \pm 0.9$ & - & - & $-3.4 \pm 0.4$ & - & - \\
\hline UGC 11262 & SF/AGN & $2.7 \pm 0.4$ & $0.1 \pm 0.2$ & $0.2 \pm 0.2$ & $-3.7 \pm 1.1$ & $8.7 \pm 0.4$ & $575 \pm 173$ \\
\hline NGC 6762 & AGN & $2.4 \pm 0.5$ & - & - & $-3.3 \pm 0.1$ & - & $234 \pm 70$ \\
\hline UGC 11680 & AGN & $2.3 \pm 0.2$ & - & - & $-2.5 \pm 0.1$ & - & - \\
\hline UGC 11694 & AGN & $3.1 \pm 0.5$ & - & - & $-3.3 \pm 0.2$ & - & $316 \pm 95$ \\
\hline UGC 11717 & AGN & $4.9 \pm 0.7$ & - & - & $-3.4 \pm 0.1$ & - & - \\
\hline UGC 11740 & SF/AGN & $2.8 \pm 0.6$ & $0.1 \pm 0.2$ & $0.5 \pm 0.2$ & $-3.7 \pm 0.5$ & $8.6 \pm 0.3$ & $34 \pm 10$ \\
\hline NGC 7549 & SF/AGN & $2.6 \pm 0.2$ & $0.4 \pm 0.2$ & $0.3 \pm 0.2$ & $-3.4 \pm 0.3$ & $8.6 \pm 0.2$ & - \\
\hline
\end{tabular}

lines available in our spectra allow us to derive the ionization parameter from the ratio $\left[\mathrm{O}_{\mathrm{II}}\right] /[\mathrm{O} \mathrm{III}]=\lambda 3727 /(\lambda 4959+\lambda 5007)$, using the relation $\log u=-0.80 \log ([\mathrm{OII}] /[\mathrm{OIII}])-3.02$, after Díaz et al. (2000). The derived ionization parameters are listed in Table 3. The reported values are in the higher range compared to most of the known HII regions (e.g. the Orion Nebula, Sánchez et al. 2007c). The main reason for these high values is most probably the presence of an AGN in a substantial fraction of the galaxies of the current sample.

Gas-phase oxygen abundances can also be obtained from the emission lines present in the CALIFA data. However, a direct oxygen abundance determination requires the presence of temperature-sensitive auroral lines such as [O III]4363 $\AA$ and/or $\left[\mathrm{N}_{\text {II }}\right] 5755 \AA$, which are only present in the low-to-intermediate metallicity regime and are generally much fainter than the corresponding nebular ones, our spectra are not deep enough to detect them, in most of the cases. Nevertheless, we can turn to strong-line methods to derive the gas metallicity (e.g., Kewley \& Ellison 2008; López-Sánchez \& Esteban 2010), bearing in mind that these calibrations are only valid for gas ionized by star formation. In the case of hard ionization due to AGN, the derivation of the oxygen abundance is largely unexplored, and only detailed photo-ionization models can be used to quantitatively estimate it (e.g. Storchi-Bergmann et al. 1998). For the purpose of the current exploratory study, we restrict ourselves to objects which are not AGN-dominated and we adopt the simple O3N2 indicator Pettini \& Pagel (2004), $12+\log (\mathrm{OH})=$ $8.73+0.32 \log _{10} \frac{[\mathrm{O} \mathrm{III}] \lambda 5007 / \mathrm{H} \beta}{[\mathrm{N} I I] \lambda 6583 / \mathrm{H} \alpha}$. The derived abundances are listed in Table 3. They are in the range of values expected for galaxies of this kind. No significant variation is found between the values reported for the central and integrated spectrum.
Table 3 lists also the electron density of the ionized gas from the $\left[\mathrm{S}_{\text {II }}\right] 6717 \AA / 6731 \AA$ doublet ratio. This particular line ratio is sensitive to the electron density only for a particular range of values (Osterbrock 1989). Only electron densities within this range are listed in Table 3. A wide range of values is found for the electron densities, indicating different gas conditions for the different objects and spatial regions.

We conclude from this section that the emission line spectra delivered by CALIFA live up to the expectations in terms of their scientific usability. Future analyses should be performed, improving the subtraction of the underlying stellar population, the treatment of dust attenuation and the derivation of the oxygen abundance.

\subsection{Spatial distribution of the spectroscopic properties}

In the previous section we have described the results of the analysis of single spectra, selected from two different apertures. A further design goal of CALIFA is, however, the analysis and comparison of the spatial distribution of those properties. We now turn towards showing the power of CALIFA in this domain.

We have applied the fitting procedure described in previous sections to each individual spectrum of each datacube, for both the V500 and V1200 datasets. For each spaxel of each datacube we thus recover the corresponding spectral information, and it is therefore possible to create two-dimensional maps for each of the derived parameters that describe the stellar populations (luminosity weighted ages, metallicities, dust attenuation, and/or coefficients of the mix of the adopted SSP templates), and the ionized gas (line intensities, dust attenuation, diagnostic line ratios, ionization strength, oxygen abundance, electron density). In 
S.F. Sánchez et al.: CALIFA, the Calar Alto Legacy Integral Field Area survey. I.

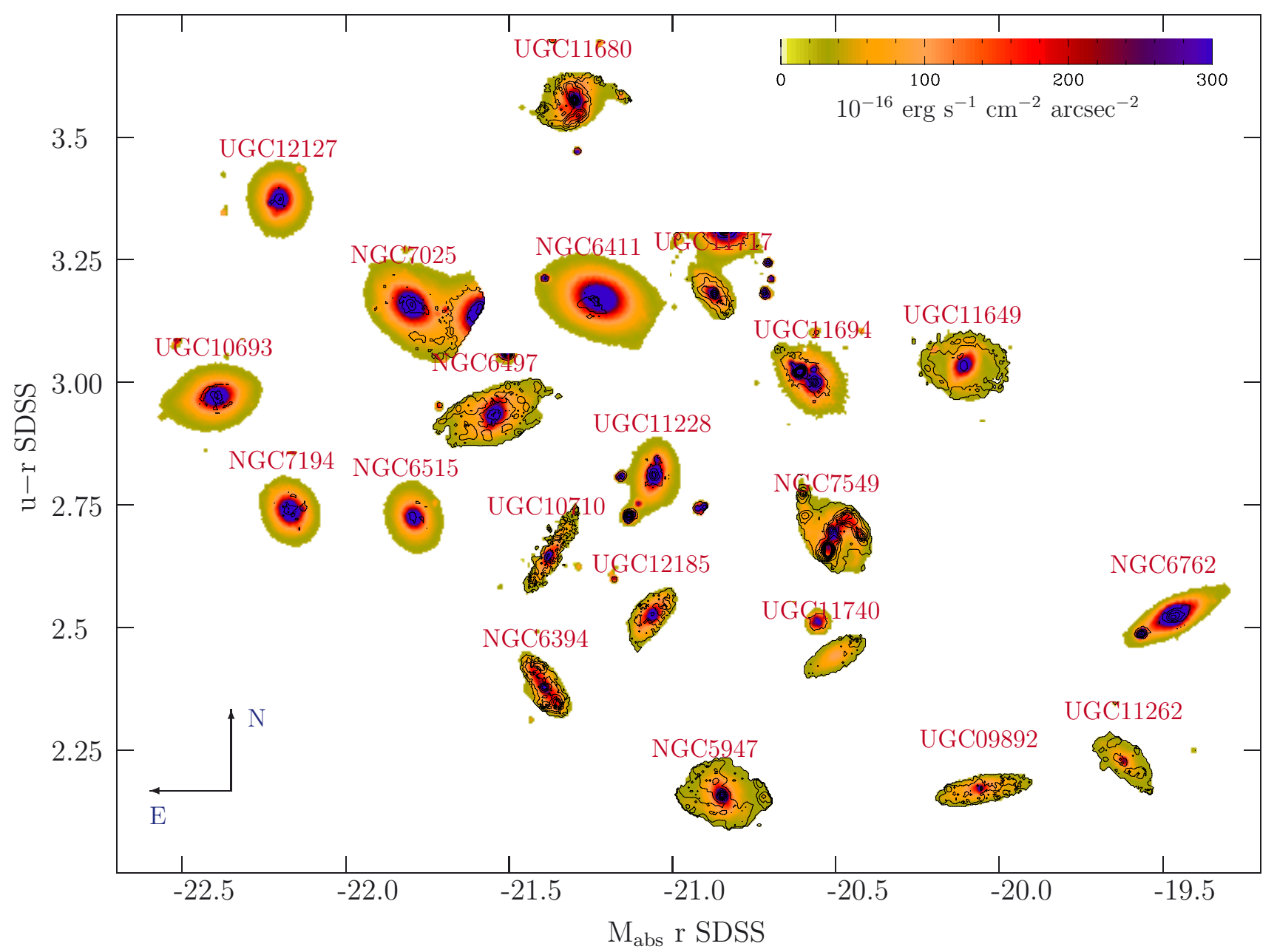

Fig. 21. Color magnitude diagram of the galaxies currently observed within CALIFA, for the SDSS $u-r$ observed colors versus the $r$-band absolute magnitude. For each galaxy we plot in color a map for the continuum intensity at $\sim 6550 \AA$ down to a surface brightness of $\sim 23 \mathrm{mag} / \mathrm{arcsec}^{2} \mathrm{AB}$ ( $>3 \sigma$ detection limit per spaxel), together with a contour plot of the $\mathrm{H} \alpha$ emission (if detected), derived by the fitting procedure described in the text (applied to the V500 data). The contours start at $0.3 \times 10^{-16} \mathrm{erg} \mathrm{s}^{-1} \mathrm{~cm}^{-2} \operatorname{arcsec}^{-2}$, and are spaced on a logarithmic scale, following the equation $f=0.3+N^{1.5}(0.3,1.3,3.1,5.5 \ldots)$. The NED name of the galaxies has been included, together with the orientation of the map on the sky. Note that some images have been shifted by up to $\pm 0.5 \mathrm{mag}$ around the nominal color-magnitude coordinate to avoid overlap. Nearby (projected) companions (e.g., NGC 7025) and foreground stars have not been masked (e.g., UGC 11694, UGC 11717).

addition, the two-dimensional kinematic structure for both the ionized gas and the stellar populations are derived. We will illustrate the results of this analysis in the next figures.

Figure 21 shows the color-magnitude diagram for the galaxies of the analyzed subsample, based on the SDSS $u-r$ observed colors and the $r$-band absolute magnitude. For each galaxy, we plot a color-map for the continuum intensity at $\sim 6550 \AA$ down to a surface brightness of $\sim 23 \mathrm{mag} / \operatorname{arcsec}^{2} \mathrm{AB}$ ( $>3 \sigma$ detection limit per spaxel), together with a contour plot of the $\mathrm{H} \alpha$ emission (if detected). The names of the galaxies have been indetified. As expected, the brightest and reddest galaxies show the least ionized gas emission. These dry or almost dry galaxies are mostly morphological early-types (E and S0). Some of them show some emission lines in the central regions. However, the detected emission in $\mathrm{H} \alpha$ is below the conservative detection limit adopted in Table 3. A more detailed decoupling analysis between the emission lines and the underlying stellar population is needed to ensure the reliability of this detection, in particular of E and S0 galaxies (Kehrig et al., ).

A greater morphological diversity and a larger range of colors is found among the galaxies with ionized gas. There are the typical face-on (e.g. NGC 5947, NGC 6497) and edge-on (e.g. UGC 10710, UGC 09892) spiral galaxies; galaxies with strong bars (e.g. NGC 6392); low surface brightness galaxies (e.g. UGC 11740), and galaxies with evidence of recent interactions (e.g. UGC 11680 and NGC 7549). The two reddest galaxies with gas (UGC 11680 and UGC 11717) both harbor an AGN and have strong dust attenuation in their integrated spectrum (as derived from the ionized gas, Table 3 ). The spatial distribution of the ionized gas also shows a wide diversity. In some galaxies, the ionized gas is concentrated in the central regions (e.g. NGC 6762, dominated by a ionization different than star formation), while in other objects the ionized gas follows the spiral arms (UGC 11680, NGC 5947 or NGC 7549), or is located in a ring (UGC 11649). The ionization source in most of these galaxies is purely star formation, based on their integrated spectra. The most distorted $\mathrm{H} \alpha$ morphology is shown by NGC 7549, which is also the galaxy with the highest SFR of all those dominated by pure star formation in the integrated spectrum. The SDSS image of this object shows the typical distorted morphology produced by an interaction and/or merging process.

The ionization conditions change considerably not only from galaxy to galaxy, but also within each galaxy. Figure 22 illustrates these changes, by showing the spatial distribution of the 


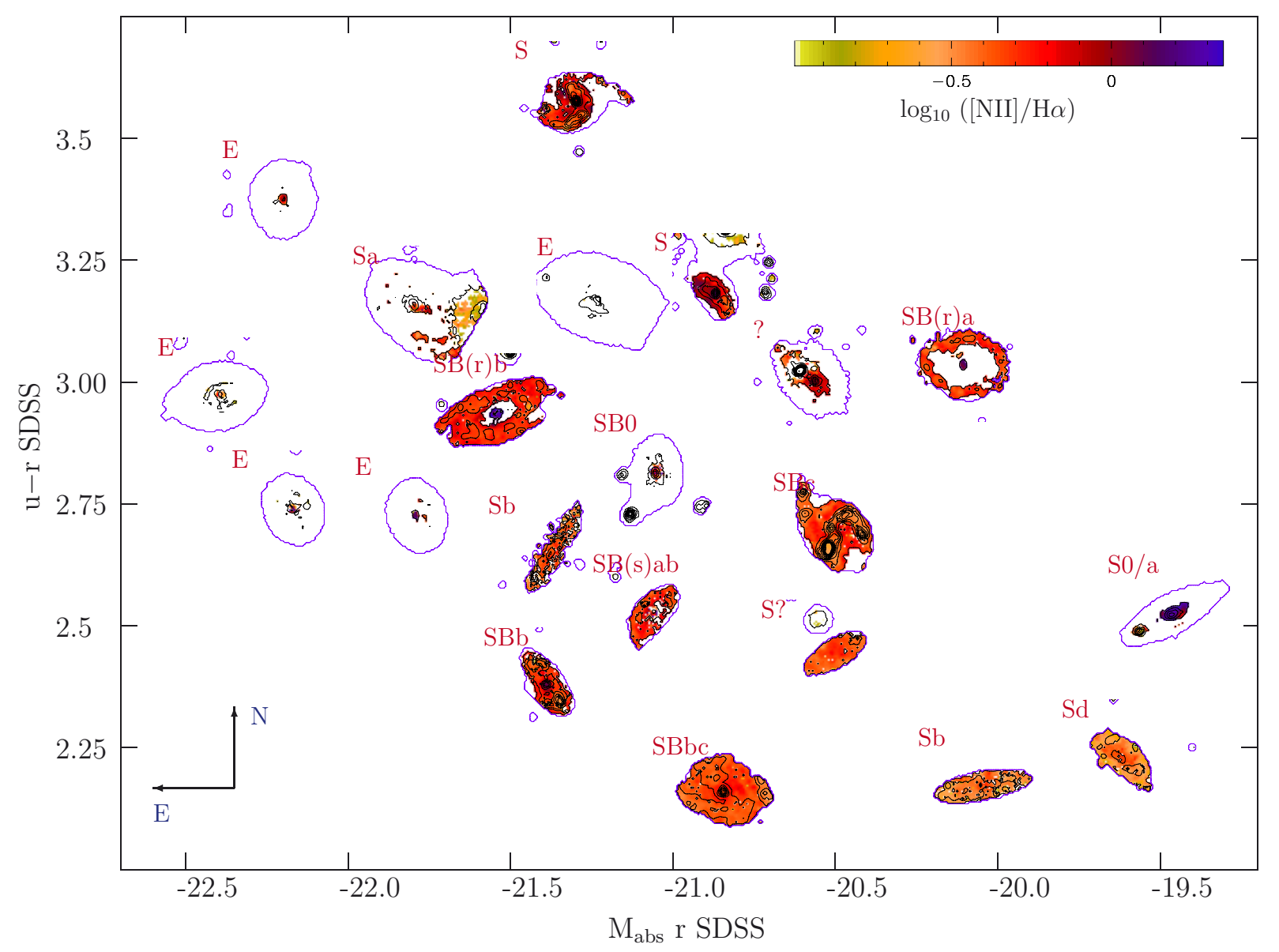

Fig. 22. Color maps showing the distribution of the emission line ratios between [NII] $\lambda 6583$ and $\mathrm{H} \alpha$, in a color-magnitude diagram similar to that of Fig. 21. In the present case the solid contours show the intensity of the $\mathrm{H} \alpha$ emission, adopting the same levels as in Fig. 21. The dashed blue contours show 3 intensity levels of the continuum emission at $\sim 6550 \AA$ (starting at $3 \times 10^{-16} \mathrm{erg} \mathrm{s}^{-1} \mathrm{~cm}^{-2} \operatorname{arcsec}^{-2}$ ). They have been included to indicate the physical extent of the continuum emission in the galaxies.

[NII] $\lambda 6583 / \mathrm{H} \alpha$ emission line ratio, for each galaxy. In Fig. 20 it was shown how this line ratio, in combination with $[\mathrm{OIII}] / \mathrm{H} \beta$, can be used to derive the main source of the ionization. In most of the ionization models, values of $\log ([\mathrm{NII}] / \mathrm{H} \alpha)>1$ cannot be produced by star formation, even in the case of very strong starbursts. Figure 22 shows that such values are only found in the central regions of some objects: NGC 6762, NGC 6497 and UGC 11649. In all these cases the ionization is clearly dominated by an AGN (Table 3). However, while in some the ionization is concentrated in the central region (NGC 6762), in other cases there are clear extended emission regions most problably ionized by the AGN (NGC 6497 and UGC 11649). Similar comparative analyses between different galaxy types and within galaxy classes can be performed using any of the parameters that characterize the ionized gas, like those listed in Table 3.

Figure 23 shows two diagnostic diagrams, similar to the ones presented in Fig. 20, corresponding to the individual spectra within the V500 datacubes of two objects, UGC 11680 and NGC 7549. These two diagrams illustrate how the ionization source changes with location, as already illustrated in Fig. 22. The track along the diagnostic diagram due to pixel-to-pixel variations in a single galaxy is an important tool to understand the nature of the ionization (e.g., Sharp \& Bland-Hawthorn 2010). In the case of UGC 11680 , there are regions corresponding to all three regimes, dominated by star-formation, intermediate and dominated by an AGN. On the other hand, the gas ionization in NGC 7549 is clearly dominated by star-formation, with a tail of $\sim 10 \%$ of the spectra showing possible intermediate ionization (e.g., Alonso-Herrero et al. 2010). In future studies these diagrams will be used to separate the different ionization sources in the spatial domain, trying to correlate them with other local properties of the galaxy.

Figure 24 shows maps of the D4000 stellar population index, sensitive to the age of the stellar component (e.g., Fig. 18), in a similar color-magnitude diagram to Fig. 21. Radial gradients are readily observed, which in some cases highlight abrupt transitions between different structural components of the galaxies (e.g. bulge, bar, disc). As expected, the morphologically earlytype galaxies (E/S0), bright, red and dry, are dominated by old stellar populations (D4000 > 2), with little variation within their optical extent. Late-type galaxies, on the other hand, show a wider variations of the age of the stellar population, both from galaxy to galaxy and within the same galaxy. In most cases there is a gradient in the ages, such that the central region is older than the outer parts. This is most evident for the face-on spirals.

A more detail analysis of the variation of the stellar populations across the field requires the comparison of either the distribution of the luminosity-weighted ages and metallicities or the corresponding indices. Figure 25 shows the distribution of the metallicity-sensitive $[\mathrm{MgFe}]$ index and the age-sensitive D4000 index, derived spaxel-to-spaxel across the field of view of the two galaxies shown in Fig. 23. Only those spaxels with an intensity above $3 \times 10^{-17} \mathrm{erg} \mathrm{s}^{-1} \mathrm{~cm}^{-2} \operatorname{arcsec}^{-2}(S / N \sim 10)$ at $\sim 5000 \AA$ have been included in the plots. Both figures illustrate 
S.F. Sánchez et al.: CALIFA, the Calar Alto Legacy Integral Field Area survey. I.
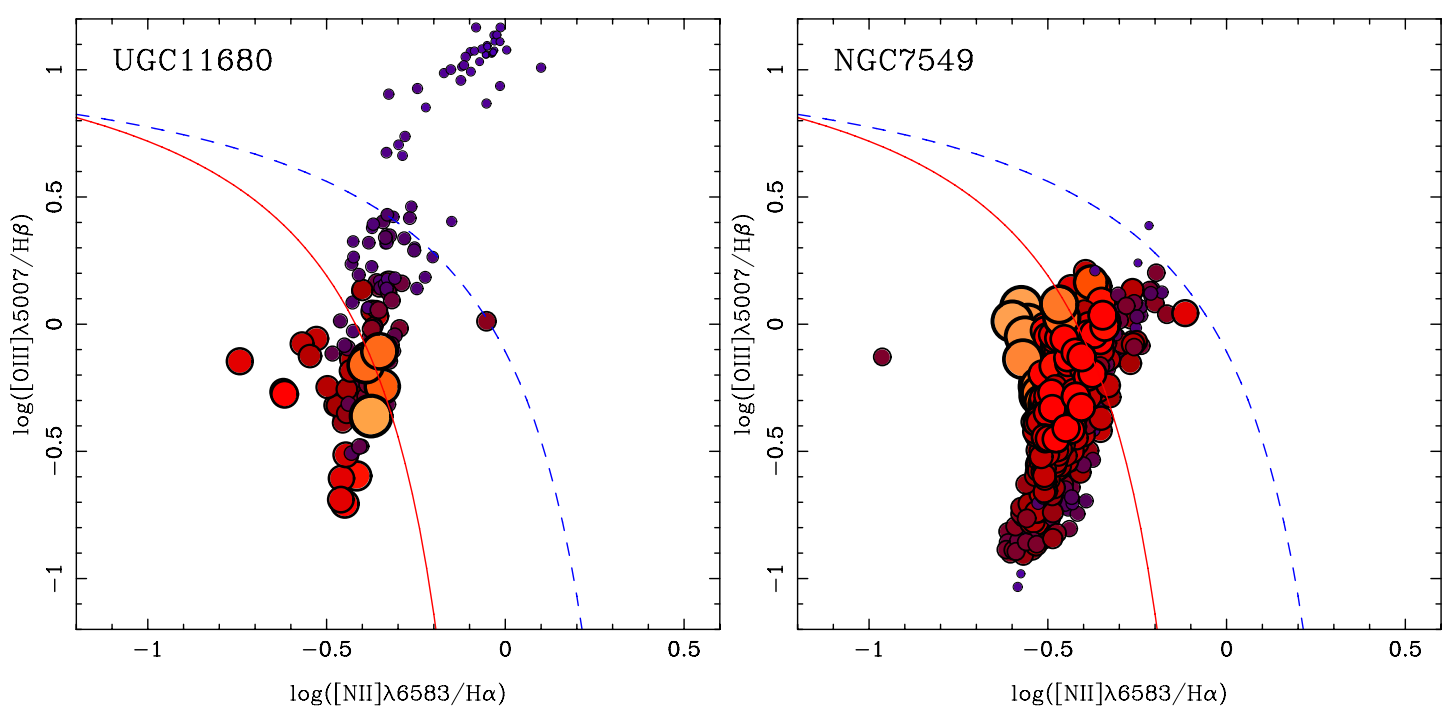

Fig. 23. [OIII] $\lambda 5007 / \mathrm{H} \beta$ vs. [NII] $\lambda 6583 / \mathrm{H} \alpha$ diagnostic diagram for the individual spectra within the V500 datacubes corresponding to two different objects, UGC 11680 (left panel) and NGC 7549 (right panel) in the currently observed CALIFA sample. The solid and dashed-lines are similar to those shown in Fig. 20. The size and colors of the symbols indicate the distance to the center of the galaxy, with bluer and smaller circles located in the inner regions and orange and larger ones located in the outer parts.

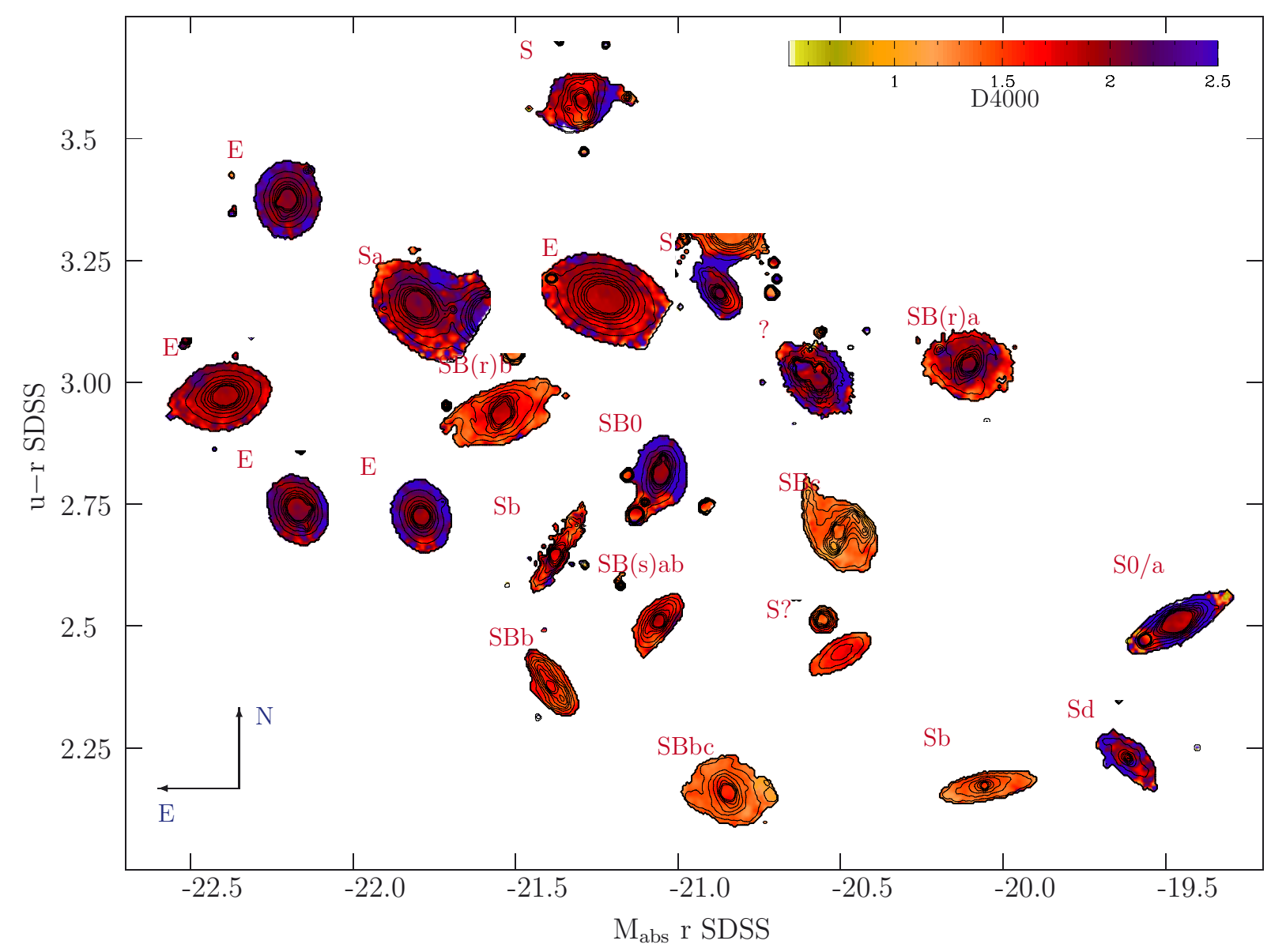

Fig. 24. Color maps showing the distribution of the D4000 stellar population index in a color-magnitude diagram similar to that of Fig. 21.

in more detail the change in the stellar populations from the inner to the outer regions.

It is interesting to note that the stellar populations of NGC 7549 show a clear gradient, with the inner regions dominated by old and metal rich stars, and the outer ones dominated by younger and metal poor ones. This is the typical trend expected in an inside-out secular evolution of a disk-dominated galaxy. On the other hand, this gradient is less evident in UGC 11680, where there is a more diverse mix of stellar populations at all radii. While both galaxies are face-on spirals with evidence of a recent interaction, the gas ionization is dominated by star-formation in most of the extension of NGC 7549, while 

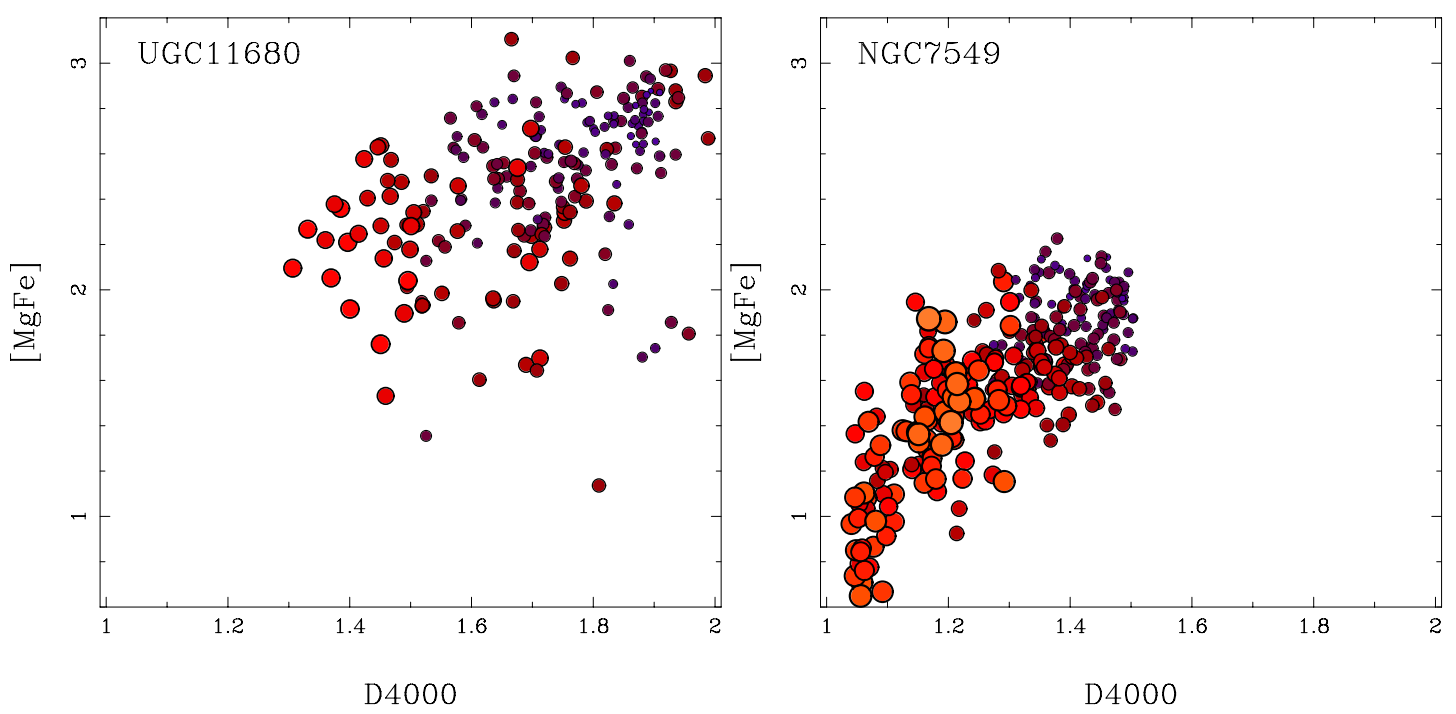

Fig. 25. Distribution of the metallicity sensitive $[\mathrm{MgFe}]$ stellar index and age sensitive D4000 index across the field of view for the two galaxies shown in Fig. 23. The colors and sizes of the circles indicate the distance from the center, defined as the intensity peak in the $V$-band. The bluer/smaller circles indicate the central regions and the orange/larger symbols the outer ones. The figure illustrates in more detail the change in the stellar populations described in Fig. 24.

UGC 11680 hosts an AGN. Whether this is related to the observed differences in the stellar populations is one of the main goals of the CALIFA survey, i.e., to study the inter-relation of the spatially resolved spectroscopic properties of different types of galaxies.

The results presented in this subsection illustrate the potential of the CALIFA data to study the spatially resolved properties of the stellar population of galaxies. We will analyze the implication of these results on our current understanding of the evolution of galaxies in forthcoming articles.

\subsection{Kinematic maps}

The stellar kinematics were derived from the V1200 dataset in the wavelength range between 3850 and $4600 \AA$ through the stellar population fitting procedure described in Sect. 7.1. Only the V1200 datacubes with a $3 \sigma$ surface brightness detection limit above $22 \mathrm{mag} / \operatorname{arcsec}^{2}$ are used. For the remaining objects, we performed a similar analysis from the corresponding V500 data, restricted to a similar wavelength range. The accuracy in the derivation of the stellar kinematics is expected to be better for the V1200 data than for the V500, in particular in the analysis of the velocity dispersion.

Figures 26 and 27 show the velocity maps derived for the stellar population and the ionized gas, respectively, for each individual galaxy within the current dataset. These velocity maps were derived based on spaxel-to-spaxel analysis, without adopting any optimal binning. A proper binning will increase the accuracy of the kinematic analysis, as described below. The velocity maps are labelled with the corresponding grating name of the data used to derive them. Similar diagrams can be constructed for the velocity dispersion maps. Combined these diagrams allow us to classify the galaxies in terms of ordered vs. random motions by identifying non-regular motions and disturbances. For the gas kinematics we have chosen here to present the $\mathrm{H} \alpha$ kinematics, since this emission line is one of the strongest. No significant differences are found when using other strong emission lines.
Obviously, the gas kinematics can only be derived in those regions (or objects) with detected emission lines. For those galaxies, mostly late-type objects, the kinematics of both the gas and the stars are dominated by rotation, with an amplitude of $\pm 150 \mathrm{~km} \mathrm{~s}^{-1}$. There are no significant differences between the gas and stellar kinematics, at least for the objects observed so far, for those galaxies for which the stellar and gas kinematics is sampled in the same physical regions: i.e., regions with high $S / N$ ionized gas and stellar continuum. In a few cases (e.g., NGC 6497 and UGC 1164), we detect high $S / N$ ionized gas in regions with low intensity continuum. For those galaxies we do not have information of the stellar kinematics derived spaxelto-spaxel in those regions where we have derived the ionized gas one. In these cases the gas kinematics fits well with the expected values from the extrapolation of the stellar kinematics to these outer regions. NGC 7025 is a special case because it is the only morphological late galaxy without detected gas emission (so far). This galaxy, apparently dry, bright and red, shows a clear rotational pattern, consistent with its morphological classification.

All galaxies without rotation are morphologically elliptical galaxies: UGC 12127, UGC 10693, NGC 6411 and NGC 6515 (4 out of the 5 elliptical galaxies in the current sample). We have no gas kinematics for these, since no emission lines were detected in these objects. On the other hand, their stellar kinematics show a patchy structure, consistent with a flat distribution, without significant gradients. Although rotation may become apparent through a more detailed analysis, making use of spatial binning approaches, we detect none for the moment.

On the other hand, the remaining early-type galaxies, NGC 7194 (E), UGC 11228(SB0) and NGC 6762(S0/a), show significant rotation. Emission lines were detected only in NGC 6762 and only in the central region, with line ratios consistent with the presence of an AGN. Particularly interesting is the case of NGC 7194, an object that does not present any morphological signature of a disk. When compared to other galaxies classified as E, e.g. NGC 6515, both objects have similar morphologies, colors, and luminosities. The continuum emission of both galaxies is dominated by old ( $>8 \mathrm{Gyr})$ and 
S.F. Sánchez et al.: CALIFA, the Calar Alto Legacy Integral Field Area survey. I.

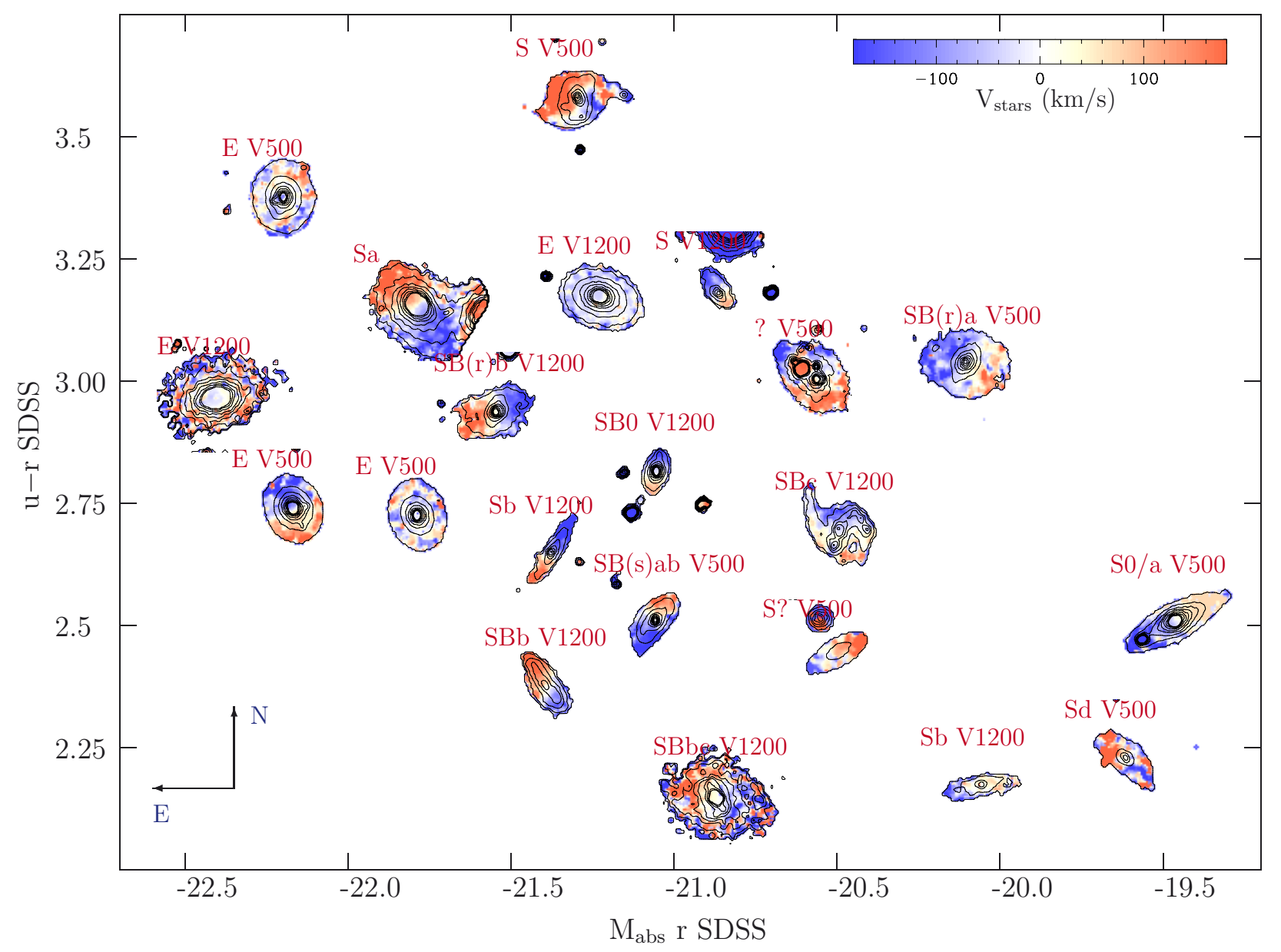

Fig. 26. Color maps showing the velocity field of the stellar component from the V1200-grating dataset in a color-magnitude diagram similar to that of Fig. 21. For those galaxies for which the $3 \sigma$ limiting surface brightness per spaxel was brighter than $22 \mathrm{mag} / \operatorname{arcsec}^{2}$, the velocity map was derived from V500 dataset. The contours show the intensity level of the continuum emission at $4000 \AA$, for the same levels as in Fig. 21.

super-solar $(Z \sim 0.028)$ stellar populations, with small or no dust attenuation $\left(A_{\mathrm{V}}<0.1 \mathrm{mag}\right)$. Despite all these similarities, both objects clearly show a different kinematic behavior. However, a full analysis of the velocity and the velocity dispersion maps is required to disentangle the real nature of the kinematic structure of these objects.

We use the high $S / N$ integrated and central spectra described above (Sect. 7.3, $S / N>40$ ) to estimate the accuracy in the derivation of the stellar velocity. First, each spectrum was split into consecutive, non overlapping wavelength ranges of $200 \AA$. Then, each sub-spectrum was fit with a multi-SSP, adopting the procedure described in Sect. 7.1. The stellar systemic velocity derived for each wavelength range is recorded. The typical standard deviations around the central values are $\sim 5 \mathrm{~km}^{-1}$ for the $\mathrm{V} 1200$ data, and $\sim 10 \mathrm{~km}^{-1}$ for the V500 one. Taking into account that $200 \AA$ is only a short wavelength range, these values agree with the expectations based on the accuracy of the wavelength calibration (Sect. 6.2).

This estimation of the accuracy of the kinematic properties may be misleading, since the $S / N$ of the individual spaxels is lower than that of the integrated and central spectra. To derive maps of the stellar kinematics, including the mean velocity and velocity dispersion we need a minimal $S / N$ per spectrum, which requires the adoption of a spatial binning technique (e.g. Emsellem et al. 2004). First, we apply an a priori cut threshold in the signal-to-noise per spaxel of $S / N>3$. Next, we perform a
Voronoi binning following Cappellari \& Copin (2003). Different experiments have been performed to derive the optimal limit of the $S / N$ goal in the binned data, regarding the accuracy of the derived parameters. For the velocity and velocity dispersion a compromise has been adopted between maximizing the $S / N$ per bin and minimizing the size of the final bins: i.e., keeping as much spatial information as possible.

Our tests indicate that to achieve a typical accuracy of $\sim 5 \mathrm{~km} \mathrm{~s}^{-1}$ in the mean velocity and a typical accuracy of $\sim 15 \mathrm{~km} \mathrm{~s}^{-1}$ in the velocity dispersion, for the V1200 data, a final $S / N$ per bin of $\sim 20$ is required. With this $S / N$ requirement, the typical bin has a projected size of a few arcseconds. Figure 28 illustrates the result of these experiments, showing, for the velocity and velocity dispersion maps, together with the estimated errors for both parameters based on an extensive Monte Carlo simulation for a particular target. At $\sim 20-30^{\prime \prime}$ from the center of the galaxy, there is a rise in the error to $\sim 30-40 \mathrm{~km} \mathrm{~s}^{-1}$. This effect is stronger in the distribution of velocity dispersions. This is due to the slight decrease of the $S / N$ per bin and the fact that in the outer regions the velocity dispersion is smaller (in general), and therefore harder to measure. This effect is shown in the simulations presented by Marmol-Queralto et al. (2011), for the CALIFA feasibility studies.

Similar results are derived for all the galaxies observed sofar, as expected due to the sample selection and survey strategy which ensures a similar $S / N$ pattern across the field for the 


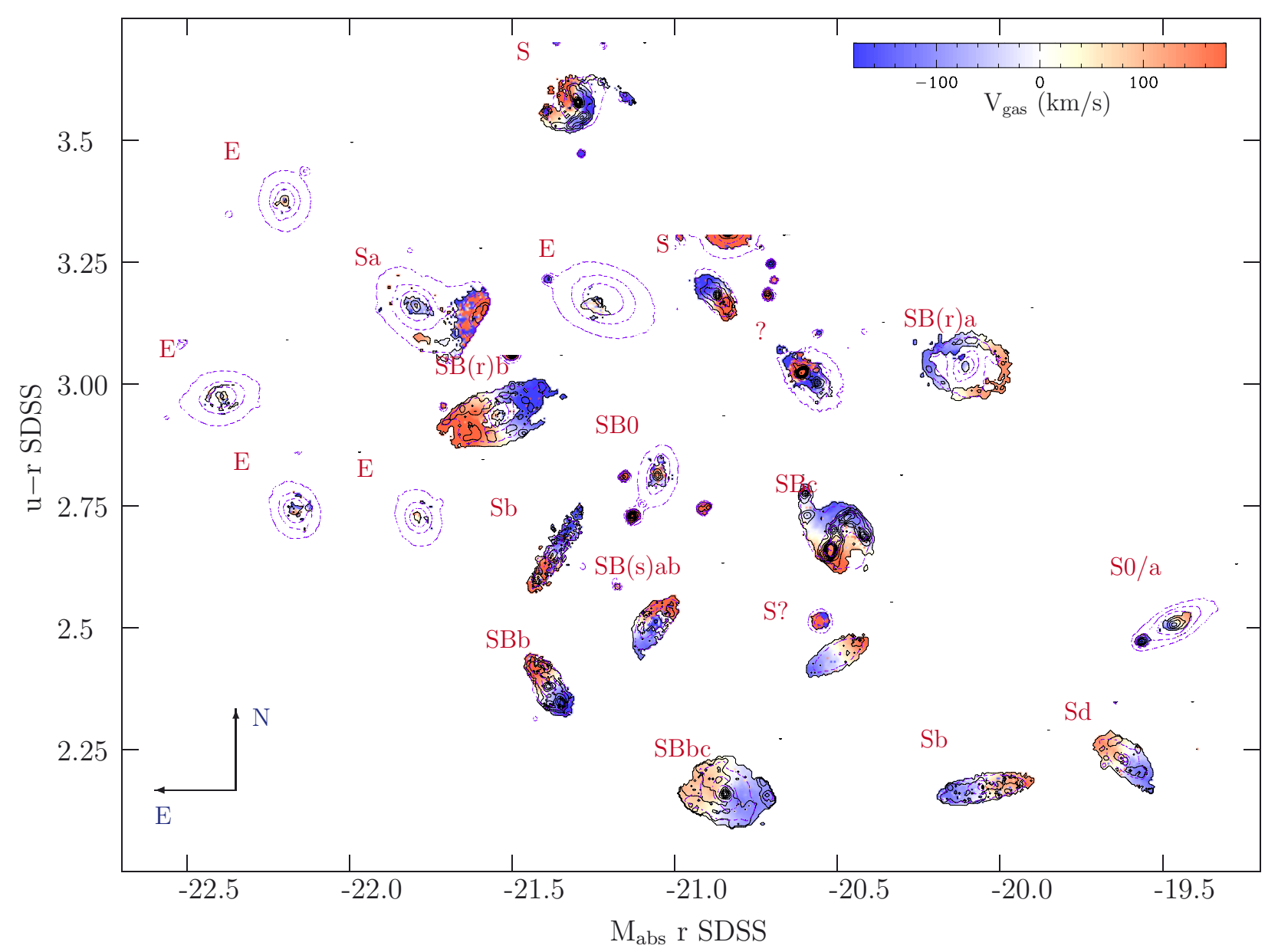

Fig. 27. Color maps showing the velocity field of the ionized gas derived from the $\mathrm{H} \alpha$ emission. The contours are similar to those shown in Fig. 22.

different targets. A more detailed report on the accuracy of the stellar kinematics will be described in forthcoming papers.

\subsection{Kinematics analysis}

As indicated in the introduction one of the goals of the current survey is to use the stars and gas kinematics to derive physical properties of the galaxies, such as dynamical mass. This requires a detailed modelling of the velocity curves (and dispersion maps) of each particular galaxy, which is outwith the scope of the current article. To probe the quality of the current data to perform these proposed studies a simple modelling of the velocity is performed. We used the maps shown in Fig. 26, trying to recover the asymptotic rotation speed (which is related to the dynamical mass), for the disk-galaxy NGC 5947.

For this study the observed rotational curve was deprojected. De-projection requires the position of the kinematics axis and the inclination angle to be known. The kinematic center was fit by eye using the continuum and $\mathrm{H} \alpha$ intensity maps, and the axes were adjusted to give the most accurate rotation curve, i.e., symmetrical and with singular velocity at the center. The inclination angle was found from the 2MASS K-band axis ratios assuming an infinitesimally thin circular disk.

This deprojected rotation curve was fit with a simple (but robust) arctan function of the form,

$v(r)=v_{\mathrm{sys}}+\frac{2 v_{\mathrm{r}}}{\pi} \arctan (s r-c)$

A8, page 28 of 31 where $v_{\text {sys }}$ is the systemic velocity of the gas and $v_{\mathrm{r}}$ is the asymptotic rotation speed of the disc, $s$ characterises the slope of $v(r)$ in the inner part of the galaxy and $c$ is the parameter that characterises any offset in the rotation axis of the galaxy. A model of the velocity map was created by re-projecting the best fitting arctan function. Figure 29 shows the original velocity map, the re-projected model and the residual map, in which the model is subtracted from the original map. The areas with noisy gas emission, excluded from the fitting analysis, have been masked from the figures. The residual map highlights the deviations from purely circular velocity. The gas velocity map of NGC 5947 is well described by a purely rotating disc, assuming an asymptotic velocity $\left(v_{\mathrm{r}} \sim 230 \mathrm{~km} \mathrm{~s}^{-1}\right)$ and an inner regions slope $\left(s \sim 0.3 \operatorname{arcsec}^{-1}\right)$. The residuals are in general lower than $30 \mathrm{~km} \mathrm{~s}^{-1}$ across the field.

The conclusion from our exploratory kinematic analysis is that the global information that CALIFA will provide will allow us not only to classify galaxies through their kinematics, but also to build rotation curves and dispersion profiles (as shown in Fig. 28), as well as detailed mass models for a large sample of galaxies of all morphological types and over a wide range of stellar mass.

\section{Conclusions}

The Calar Alto Legacy Integral Field Area survey (CALIFA survey), has been designed to be the first survey to provide IFS data for a statistical sample of all galaxy types in the local Universe. 

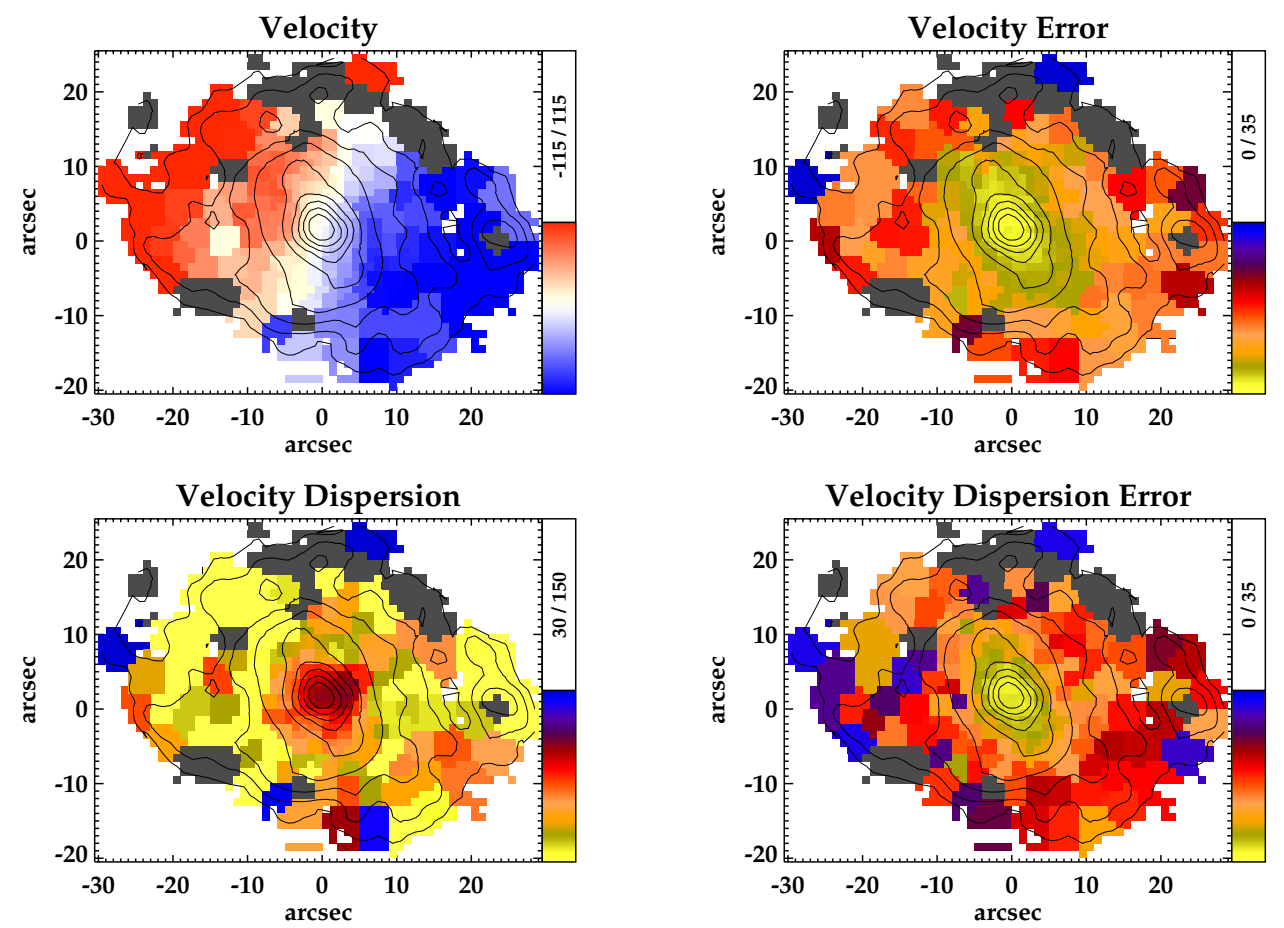

Fig. 28. Results from the stellar kinematics analysis. From left-top to right-bottom the binned maps corresponding to the derived stellar velocity, its error, the stellar velocity dispersion and its corresponding error, all in units of $\mathrm{km} \mathrm{s}^{-1}$. Values with errors larger than $35 \mathrm{~km} \mathrm{~s}^{-1}$ have been masked-out, and indicated with grey-colors.
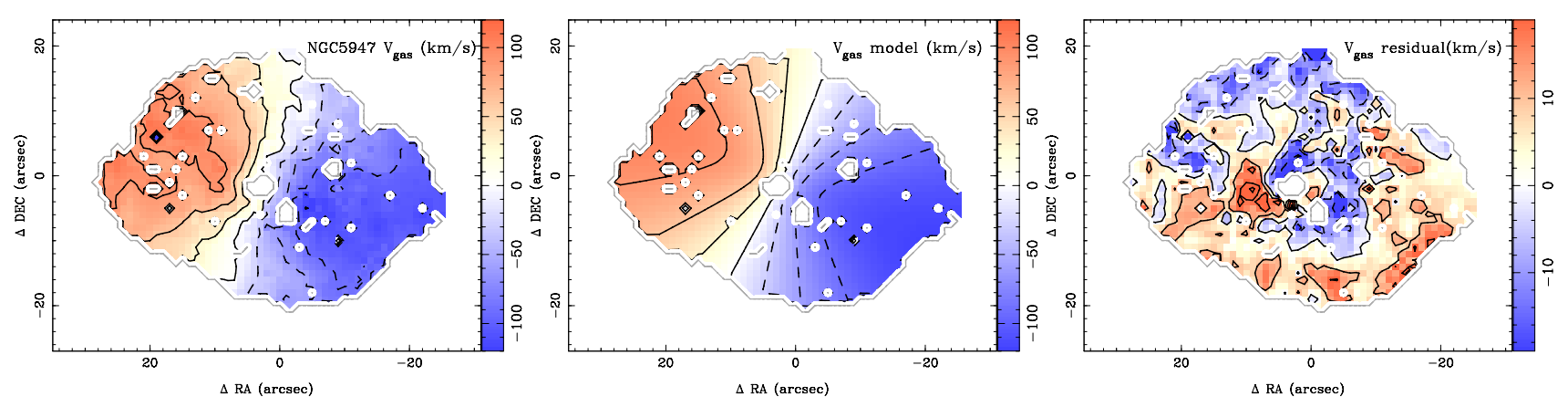

Fig. 29. Results from the gas kinematics analysis. The left panel shows the H $\alpha$ velocity map of NGC 5947 shown in Fig. 27. The central panel shows the best-fit model assuming a simple arctan-rotational curve. Finally the right panel shows the residual after subtracting the model to the velocity map. The spaxels not used in the fitting procedure have been masked. Contours indicate different velocity levels, with constant step of $30 \mathrm{~km} \mathrm{~s}^{-1}$ in the first two panels, and $10 \mathrm{~km} \mathrm{~s}^{-1}$ in the last one.

On completion it will be the largest and the most comprehensive wide-field IFU survey of galaxies carried out to date. It will thus provide a valuable bridge between large single aperture surveys and more detailed studies of individual galaxies.

In the first part of this paper we have presented the survey design, the data reduction pipeline and a number of quality control procedures. We have reached the following conclusions:

- At completion the survey will yield a statistically significant sample of $\sim 600$ galaxies, which are representative of the galaxy population in the local Universe.

- Already at this early stage in the survey we have a working data reduction pipeline, which operates without human intervention. We will be able to improve it regularly and provide consistently re-reduced complete datasets to the community.

- To give the community a feeling for the quality of data that CALIFA will deliver, we have compared the CALIFA spectra to the more generally known SDSS spectra for equivalently sized central regions of those galaxies that are in common between both datasets. From our preliminary analysis we find that the information content of the CALIFA spectra is at least equivalent to those of the SDSS (for a matched aperture).

- We have presented the main quality parameters of the dataset: depth, spectrophotometric accuracy and stability, wavelength calibration and final spectral resolution. The analysis of the quality of the currently observed objects indicates that we reach the target depth for the survey in $75 \%$ of the datacubes and that the loss of depth in the remaining cases can be clearly attributed to adverse observing conditions. From all our quality checks we conclude that we are on track for providing high-quality, well-calibrated and well-characterized reduced data to the users of the CALIFA legacy survey. 
In the second part of the paper we have presented an exploratory analysis of the first set of 21 galaxies observed through 2010 in order to verify the scientific usability of the data. We reached the following conclusions:

- We have shown that the absorption line spectra yield information on ages (and therefore mass-to-light ratios) and metallicities of the stellar population. We expect that with a more stringent analysis we will also be able to recover abundance ratios for the $\alpha$ elements.

- We have shown that the CALIFA emission line spectra will yield dust attenuation, star formation rates, excitation mechanisms and ionization parameters, metallicities and electron densities.

- The conclusion from our exploratory kinematic analysis is that the global information from CALIFA will allow us to not only classify galaxies through their kinematics, but will also allow us to build rotation curves and dispersion profiles, as well as detailed mass models for a large sample of galaxies of all morphological types and over a wide range of stellar mass.

- CALIFA will allow us to quantify the effects of sampling different physical aperture sizes. As an example we have shown that the fraction of galaxies classified as star forming (vs. AGN) is larger for integrated spectra than for central spectra. This is of course expected. CALIFA, however, will allow us to quantify these effects.

- Finally, we have shown how comparative analyses between different galaxy types and within individual galaxies can be performed using any of the derived parameters. CALIFA will thus be the first survey allowing comparative studies of the $2 \mathrm{D}$ distribution of galaxy properties in a statistically meaningful way, including galaxies of many types.

With two-dimensional maps of all these observables in hand, we expect that the data from the CALIFA survey will 1) characterize the local galaxy population in a way that will constitute a benchmark for models and other datasets alike and 2) will produce qualitatively new results on many topics of current active research, such as the build-up of galaxy disks, the influence of environment, galaxy bi-modality (morphology, kinematics, stellar populations) and possible transition objects (green valley), comparative studies of AGN and non-AGN galaxies, kinematic galaxy classification, stellar vs. dark mass, the origin and evolution of the warm ISM, to name but a few.

CALIFA data will become public in regular data releases and we very much hope that the community will actively use the data for their own projects - only then will CALIFA have fulfilled its main goal, namely to be a legacy survey.

Acknowledgements. We thank the referee Eric Emsellem for his detailed comments which helped to improve the content and presentation of the article. We thank the director of CEFCA, Dr. M. Moles, for his sincere support to this project. We thank the Viabilidad, Diseno, Acceso y Mejora funding program, ICTS-2009-10, and the Plan Nacional de Investigación y Desarrollo funding program, AYA2010-22111-C03-03, of the Spanish Ministerio de Ciencia e Innovacion, for the support given to this project. I.M. and J.M. acknowledge financial support from the Spanish grant AYA2010-15169 and Junta de Andalucía TIC114 and Excellence Project P08-TIC-03531. C.K., as a Humboldt Fellow, acknowledges support from the Alexander von Humboldt Foundation, Germany. B. Jungwiert acknowledges support by the grants AV0Z10030501 (Academy of Sciences of the Czech Republic) and LC06014 (Center for Theoretical Astrophysics, Czech Ministry of Education). T. Bartáková acknowledges support by the grants No. 205/08/H005 (Czech Science Foundation) and MUNI/A/0968/2009 (Masaryk University in Brno). Polychronis Papaderos is supported by a Ciencia 2008 contract, funded by FCT/MCTES (Portugal) and POPH/FSE (EC). This paper makes use of the Sloan Digital Sky Survey data. Funding for the SDSS and SDSS-II has been provided by the Alfred P. Sloan Foundation, the Participating Institutions, the National Science
Foundation, the U.S. Department of Energy, the National Aeronautics and Space Administration, the Japanese Monbukagakusho, the Max Planck Society, and the Higher Education Funding Council for England. The SDSS Web Site is http://www.sdss.org/. The SDSS is managed by the Astrophysical Research Consortium for the Participating Institutions. The Participating Institutions are the American Museum of Natural History, Astrophysical Institute Potsdam, University of Basel, University of Cambridge, Case Western Reserve University, University of Chicago, Drexel University, Fermilab, the Institute for Advanced Study, the Japan Participation Group, Johns Hopkins University, the Joint Institute for Nuclear Astrophysics, the Kavli Institute for Particle Astrophysics and Cosmology, the Korean Scientist Group, the Chinese Academy of Sciences (LAMOST), Los Alamos National Laboratory, the Max-PlanckInstitute for Astronomy (MPIA), the Max-Planck-Institute for Astrophysics (MPA), New Mexico State University, Ohio State University, University of Pittsburgh, University of Portsmouth, Princeton University, the United States Naval Observatory, and the University of Washington.

\section{References}

Alonso-Herrero, A., García-Marín, M., Rodríguez Zaurín, J., et al. 2010, A\&A, 522, A7

Arribas, S., Mediavilla, E., García-Lorenzo, B., del Burgo, C., \& Fuensalida, J. J. 1999, A\&AS, 136, 189

Arribas, S., Colina, L., Monreal-Ibero, A., et al. 2008, A\&A, 479, 687

Baillard, A., Bertin, E., de Lapparent, V., et al. 2011, A\&A, 532, A74

Baldwin, J. A., Phillips, M. M., \& Terlevich, R. 1981, PASP, 93, 5

Benítez, N., Gaztañaga, E., Miquel, R., et al. 2009, ApJ, 691, 241

Binette, L., Magris, C. G., Stasińska, G., \& Bruzual, A. G. 1994, A\&A, 292, 13

Blanc, G. A., Gebhardt, K., Heiderman, A., et al. 2010, in ASP Conf. Ser., 432, ed. L. M. Stanford, J. D. Green, L. Hao, \& Y. Mao, 180

Blanton, M. R., Schlegel, D. J., Strauss, M. A., et al. 2005, AJ, 129, 2562

Brinchmann, J., Charlot, S., White, S. D. M., et al. 2004, MNRAS, 351, 1151

Cappellari, M., \& Copin, Y. 2003, MNRAS, 342, 345

Cappellari, M., \& Emsellem, E. 2004, PASP, 116, 138

Cappellari, M., Emsellem, E., Krajnović, D., et al. 2011, MNRAS, 413, 813

Cardelli, J. A., Clayton, G. C., \& Mathis, J. S. 1989, ApJ, 345, 245

Castillo-Morales, A., Gallego, J., Pérez-Gallego, J., et al. 2010, MNRAS, 1804

Cid Fernandes, R., Mateus, A., Sodré, L., Stasińska, G., \& Gomes, J. M. 2005, MNRAS, 358, 363

Cid Fernandes, R., Stasińska, G., Schlickmann, M. S., et al. 2010, MNRAS, 403, 1036

Davies, J. I., Phillipps, S., \& Disney, M. J. 1990, MNRAS, 244, 385

de Jong, R. S., \& van der Kruit, P. C. 1995, VizieR Online Data Catalog, 4106, 60451

de Zeeuw, P. T., Bureau, M., Emsellem, E., et al. 2002, MNRAS, 329, 513

Díaz, A. I., Castellanos, M., Terlevich, E., \& Luisa García-Vargas, M. 2000, MNRAS, 318, 462

Ellis, S. C., Driver, S. P., Allen, P. D., et al. 2005, MNRAS, 363, 1257

Emsellem, E., Bacon, R., Monnet, G., \& Poulain, P. 1996, A\&A, 312, 777

Emsellem, E., Cappellari, M., Peletier, R. F., et al. 2004, MNRAS, 352, 721

Epinat, B., Amram, P., Balkowski, C., \& Marcelin, M. 2010, MNRAS, 401, 2113

Filippenko, A. V. 1982, PASP, 94, 715

Folkes, S., Ronen, S., Price, I., et al. 1999, MNRAS, 308, 459

Gallazzi, A., Charlot, S., Brinchmann, J., White, S. D. M., \& Tremonti, C. A. 2005, MNRAS, 362, 41

Gómez, P. L., Nichol, R. C., Miller, C. J., et al. 2003, ApJ, 584, 210

Kauffmann, G., Heckman, T. M., Tremonti, C., et al. 2003, MNRAS, 346, 1055

Kelz, A., Verheijen, M. A. W., Roth, M. M., et al. 2006, PASP, 118, 129

Kennicutt, Jr., R. C. 1998, ApJ, 498, 541

Kewley, L. J., \& Ellison, S. L. 2008, ApJ, 681, 1183

Kewley, L. J., Dopita, M. A., Sutherland, R. S., Heisler, C. A., \& Trevena, J. 2001, ApJ, 556, 121

Kewley, L. J., Jansen, R. A., \& Geller, M. J. 2005, PASP, 117, 227

Koleva, M., Prugniel, P., Bouchard, A., \& Wu, Y. 2009, A\&A, 501, 1269

Kuntschner, H., Emsellem, E., Bacon, R., et al. 2006, MNRAS, 369, 497

Le Fèvre, O., Vettolani, G., Paltani, S., et al. 2004, A\&A, 428, 1043

Lilly, S. J., Le Fèvre, O., Renzini, A., et al. 2007, ApJS, 172, 70

López-Sánchez, Á. R., \& Esteban, C. 2010, A\&A, 517, A85

MacArthur, L. A., Courteau, S., Bell, E., \& Holtzman, J. A. 2004, ApJS, 152, 175

MacArthur, L. A., González, J. J., \& Courteau, S. 2009, MNRAS, 395, 28

Marmol-Queralto, E., Sánchez, S. F., Marino, R. A., et al. 2011, A\&A, 534, A8

Moles, M., Benítez, N., Aguerri, J. A. L., et al. 2008, AJ, 136, 1325

Moro, D., \& Munari, U. 2000, A\&AS, 147, 361

Moustakas, J., \& Kennicutt, Jr., R. C. 2006, ApJS, 164, 81

Ocvirk, P., Pichon, C., Lançon, A., \& Thiébaut, E. 2006, MNRAS, 365, 46

Oke, J. B. 1990, AJ, 99, 1621 
Osterbrock, D. E. 1989, Astrophysics of gaseous nebulae and active galactic nuclei (University Science Books)

Pérez-Gallego, J., Guzmán, R., Castillo-Morales, A., et al. 2010, MNRAS, 402, 1397

Pettini, M., \& Pagel, B. E. J. 2004, MNRAS, 348, L59

Rix, H., Barden, M., Beckwith, S. V. W., et al. 2004, ApJS, 152, 163

Rosales-Ortega, F. F., Kennicutt, R. C., Sánchez, S. F., et al. 2010, MNRAS, 405, 735

Roth, M. M., Seydack, M., Bauer, S.-M., \& Laux, U. 1997, in SPIE Conf. Ser. 2871, ed. A. L. Ardeberg, 1235

Roth, M. M., Bauer, S.-M., Dionies, F., et al. 1998, in SPIE Conf. Ser. 3355, ed. S. D'Odorico, 798

Roth, M. M., Becker, T., Kelz, A., \& Schmoll, J. 2004, ApJ, 603, 531

Roth, M. M., Kelz, A., Fechner, T., et al. 2005, PASP, 117, 620

Roth, M. M., Fechner, T., Wolter, D., et al. 2010, in SPIE Conf. Ser. 7742

Sánchez, S. F. 2006, Astron. Nachr., 327, 850

Sánchez, S. F., Jahnke, K., Wisotzki, L., et al. 2004, ApJ, 614, 586

Sánchez, S. F., García-Lorenzo, B., Jahnke, K., et al. 2006, New Aron. Rev., 49, 501

Sánchez, S. F., Aceituno, J., Thiele, U., Pérez-Ramírez, D., \& Alves, J. 2007a, PASP, 119, 1186

Sánchez, S. F., Cardiel, N., Verheijen, M. A. W., Pedraz, S., \& Covone, G. 2007b, MNRAS, 376, 125

Sánchez, S. F., Cardiel, N., Verheijen, M. A. W., et al. 2007c, A\&A, 465, 207

Sánchez, S. F., Rosales-Ortega, F. F., Kennicutt, R. C., et al. 2011, MNRAS, 410, 313

Sandin, C., Becker, T., Roth, M. M., et al. 2010, A\&A, 515, 35

Sarzi, M., Falcón-Barroso, J., Davies, R. L., et al. 2006, MNRAS, 366, 1151

Sarzi, M., Shields, J. C., Schawinski, K., et al. 2010, MNRAS, 402, 2187

Scoville, N., Aussel, H., Brusa, M., et al. 2007, ApJS, 172, 1

Sharp, R. G., \& Bland-Hawthorn, J. 2010, ApJ, 711, 818

Shepard, D. 1968, Proc. 1968 ACM National Conference, 1, 517

Stasińska, G., Vale Asari, N., Cid Fernandes, R., et al. 2008, MNRAS, 391, L29

Storchi-Bergmann, T., Schmitt, H. R., Calzetti, D., \& Kinney, A. L. 1998, AJ, 115,909

Strateva, I., Ivezić, Ž., Knapp, G. R., et al. 2001, AJ, 122, 1861

Trager, S. C., Faber, S. M., Worthey, G., \& González, J. J. 2000, AJ, 119, 1645

Trinchieri, G., \& di Serego Alighieri, S. 1991, AJ, 101, 1647

van Dokkum, P. G. 2001, PASP, 113, 1420

Vazdekis, A., Sánchez-Blázquez, P., Falcón-Barroso, J., et al. 2010, MNRAS, 404, 1639

Veilleux, S., \& Osterbrock, D. E. 1987, ApJS, 63, 295

Verheijen, M. A. W., Bershady, M. A., Andersen, D. R., et al. 2004, Astron. Nachr., 325, 151

Walcher, C. J., Böker, T., Charlot, S., et al. 2006, ApJ, 649, 692

Walcher, J., Groves, B., Budavári, T., \& Dale, D. 2011, Ap\&SS, 331, 1

Wisotzki, L., Becker, T., Christensen, L., et al. 2003, A\&A, 408, 455

Wolf, C., Meisenheimer, K., Rix, H., et al. 2003, A\&A, 401, 73

York, D. G., Adelman, J., Anderson, Jr., J. E., et al. 2000, AJ, 120, 1579

Zibetti, S., Charlot, S., \& Rix, H. 2009, MNRAS, 400, 1181

1 Centro Astronómico Hispano Alemán, Calar Alto, (CSIC-MPG), C/Jesús Durbán Remón 2-2, 04004 Almeria, Spain e-mail: sanchez@caha.es

2 Institute of Astronomy, University of Cambridge, Madingley Road, Cambridge CB3 0HA, UK

3 Departamento de Astrofísica y CC. de la Atmósfera, Universidad Complutense de Madrid, Madrid 28040, Spain

4 Max Planck Institute for Astronomy, Königstuhl 17, 69117 Heidelberg, Germany

5 Instituto de Astrofísica de Andalucía (CSIC), Camino Bajo de Huetor, s/n, 18008, Granada, Spain
6 Leibniz-Institut für Astrophysik Potsdam (AIP), An der Sternwarte 16, 14482 Potsdam, Germany

7 Sydney Institute for Astronomy, School of Physics A28, University of Sydney, 2006 NSW, Australia

8 Australian Astronomical Observatory, PO BOX 296, Epping, 1710 NSW, Australia

9 Instituto de Astrofísica de Canarias (IAC), Glorieta de la Astronomía S/N, La Laguna, S/C de Tenerife, Spain

10 Departamento de Fisica Teorica, Universidad Autonoma de Madrid, Cantoblanco, 28049 Madrid, Spain

11 Kapteyn Astronomical Institute, University of Groningen, 9700 AV, Groningen, The Netherlands

12 Instituto de Física de Cantabria, CSIC-UC, Avenida de los Castros S/N, 39005 Santander, Spain

13 ZAH, Landessternwarte, Königstuhl 12, 69117 Heidelberg, Germany

14 Dark Cosmology Centre, Niels Bohr Institute, University of Copenhagen, Juliane Maries Vej 30, 2100 Copenhagen, Denmark

15 Centro de Astrofísica and Faculdade de Ciências, Universidade do Porto, Rua das Estrelas, 4150-762 Porto, Portugal

16 Astronomical Institute of the Academy of Sciences of the Czech Republic, v.v.i., Bocni II 1401, 14131 Prague, Czech Republic

17 CENTRA - Centro Multidisciplinar de Astrofísica, Instituto Superior Técnico, Av. Rovisco Pais 1, 1049-001 Lisbon, Portugal

18 SUPA, Institute for Astronomy, University of Edinburgh, Royal Observatory, Blackford Hill, Edinburgh EH9 3HJ, UK

19 Laboratoire d'Astropysique de Marseille, UMR 6110 CNRS, 38 rue F. Joliot-Curie, 13388 Marseille, France

20 Department of Systems Engineering and Computing, University of Zaragoza, Teruel 44003, Spain

21 Department of Electronic Engineeing and Communications, University of Zaragoza, Teruel 44003, Spain

22 University of Vienna, Türkenschanzstrasse 17, 1180 Vienna, Austria

23 Department of Physics and Astronomy, Macquarie University, NSW 2109, Australia

24 Centro de Estudios de Física del Cosmos de Aragón (CEFCA), C/ G. Pizarro, 1, Teruel, Spain

25 Astronomical Institute of the Ruhr-University Bochum Universitaetsstr. 150, 44801 Bochum, Germany

26 Department of Physics, University of Missouri-Kansas City, Kansas City, MO 64110, USA.

27 Dep. Fysica Terica y del Cosmos, Campus de Fuentenueva, Universidad de Granada, 18071 Granada, Spain

28 Depto. Astrofisica, Universidad de La Laguna (ULL), 38206 La Laguna, Tenerife, Spain

29 Astronomisches Rechen Institut, Zentrum fuer Astronomie der Universitaet Heidelberg, Moenchhofstrasse 12-14, 69120 Heidelberg, Germany

30 Institut d'Astrophysique de Paris, CNRS, 98bis Boulevard Arago, 75014 Paris, France

31 Laboratoire GEPI, Observatoire de Paris, CNRS-UMR8111, Univ. Paris-Diderot, 5 place Jules Janssen, 92195 Meudon, France

32 Tianjin Astrophysics Center, Tianjin Normal University, 300387 Tianjin, PR China

33 Astronomical Institute, Faculty of Mathematics and Physics, Charles University in Prague, Ke Karlovu 3, 12116 Prague, Czech Republic

34 Dept. of Theoretical Physics and Astrophysics, Faculty of Science, Masaryk University, Kotlářská 2, 61137 Brno, Czech Republic 